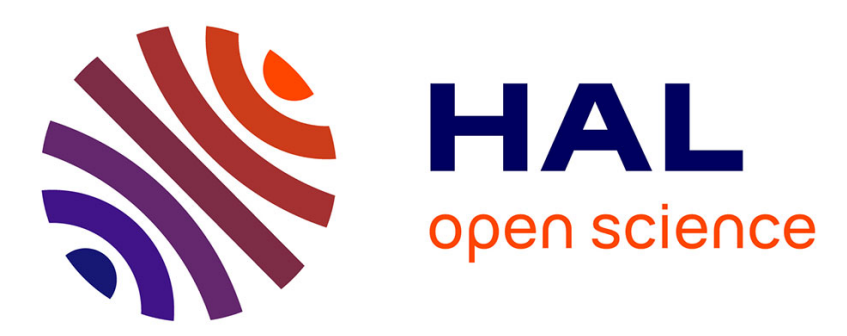

\title{
Models, Feedback Control, and Open Problems of 3D Bipedal Robotic Walking
}

Jessy W Grizzle, Christine Chevallereau, Ryan W. Sinnet, Aaron D. Ames

\section{To cite this version:}

Jessy W Grizzle, Christine Chevallereau, Ryan W. Sinnet, Aaron D. Ames. Models, Feedback Control, and Open Problems of 3D Bipedal Robotic Walking. Automatica, 2014, 50 (8), pp.1955-1988. 10.1016/j.automatica.2014.04.021 . hal-01676474

\section{HAL Id: hal-01676474 https://hal.science/hal-01676474}

Submitted on 5 Jan 2018

HAL is a multi-disciplinary open access archive for the deposit and dissemination of scientific research documents, whether they are published or not. The documents may come from teaching and research institutions in France or abroad, or from public or private research centers.
L'archive ouverte pluridisciplinaire HAL, est destinée au dépôt et à la diffusion de documents scientifiques de niveau recherche, publiés ou non, émanant des établissements d'enseignement et de recherche français ou étrangers, des laboratoires publics ou privés. 


\title{
Models, Feedback Control, and Open Problems of 3D Bipedal Robotic Walking *
}

\author{
J.W. Grizzle ${ }^{\mathrm{a}}$ Christine Chevallereau ${ }^{\mathrm{b}}$ Ryan W. Sinnet and Aaron D. Ames ${ }^{\mathrm{c}}$ \\ ${ }^{a}$ Control Systems Laboratory, EECS Department, University of Michigan, USA, (email: grizzle@umich.edu) \\ ${ }^{\mathrm{b}}$ LUNAM Université, IRCCyN, CNRS, France, (email: Christine.Chevallereau@irccyn.ec-nantes.fr) \\ ${ }^{\mathrm{c}}$ Department of Mechanical Engineering, Texas AछßM University, USA (email: \{rsinnet,aames\}@tamu.edu)
}

\begin{abstract}
The fields of control and robotics are working toward the development of bipedal robots that can realize walking motions with the stability and agility of a human being. Dynamic models for bipeds are hybrid in nature. They contain both continuous and discrete elements, with switching events that are governed by a combination of unilateral constraints and impulse-like forces that occur at foot touchdown. Control laws for these machines must be hybrid as well. The goals of this paper are fourfold: highlight certain properties of the models which greatly influence the control law design; overview the literature; present two control design approaches in depth; and indicate some of the many open problems.
\end{abstract}

Key words: Bipedal robotic locomotion; Feedback control methods; Geometric approaches; Hybrid modes; Nonlinear control systems; Periodic motion; Robots

\section{Introduction}

This paper seeks to provide control researchers with an entry point into the area of bipedal locomotion, more specifically, $3 \mathrm{D}$ bipedal walking. The emphasis is on models and control laws for achieving the simplest possible behavior, namely, asymptotically stable, periodic, walking on flat ground. This is already a very challenging and rich problem due to the multi-phase, hybrid nature of legged locomotion and the unilateral constraints that must be satisfied by the forces and torques at the foot-ground interface. Any researcher mastering this basic problem can be assured that there is plenty more to do in terms of investigating aperiodic gaits, non-flat ground, maneuvering, running, energy efficiency, autonomy, and much more.

Section 2 presents a class of hybrid models that are ubiquitous in controlled bipedal locomotion. The emphasis here is on closed-loop systems, periodic orbits, which

\footnotetext{
* The work of J.W. Grizzle is supported by NSF Grants ECCS-1343720 and ECCS-1231171. The work of C. Chevallereau is supported by ANR grants for the R2A2 project. The work of A. D. Ames is supported by NSF grant CNS-0953823. The work of R. W. Sinnet is supported by NSF through a Graduate Research Fellowship.
}

will correspond to periodic walking gaits, and their stability. The method of Poincare for establishing stability properties of periodic orbits of hybrid systems is developed with an aim at being able to apply it to highdimensional models. One of the great advantages of the method of Lyapunov for analyzing the stability properties of equilibria in nonlinear systems is that one does not need to compute a solution of the model. In the case of periodic orbits, computing solutions seems to be unavoidable and hence taking advantage of invariance and time-scale properties to simplify the analysis becomes even more essential.

Section 3 on modeling is perhaps the most important part of the paper for a control engineer. The model of a bipedal robot differs from the model of a robotic manipulator precisely because the latter is bolted to the ground while the former is not. A foot remains flat on the ground without slipping only when the reaction forces and torques at the interface satisfy strict inequalities; in particular, the normal component of the ground reaction force must be positive, as the ground cannot "pull" against a foot as a bolt will for a manipulator; in addition, tangential forces must lie in a friction cone. These restrictions are obvious, but are nevertheless ignored in many publications. Getting the dynamic models right is crucial if a proposed control solution is to be taken seri- 
ously by the robotics community. Other interesting and challenging features of bipedal models depend on the gait that is being studied. A human gait, for example, has phases where the foot is in rotation about one of its edges. This occurs because foot-ground contact is typically initiated with a heel strike, followed by the foot rotating about the heel until the sole of the foot is flat on the ground. The end of the step is typically initiated by the foot rolling up on the toe prior to being lifted from the ground to begin the swing phase. The nature of the foot-ground interface also determines the degree of actuation (or underactuation) of the corresponding dynamic model. A foot in rotation necessarily leads to underactuation, while if the foot is flat on the ground and all joints of the robot are independently actuated, the model is typically fully actuated. Most of the wellknown humanoid robots use large feet and a flat-footed walking gait in order to avoid the control complications arising from underactuation; on the other hand, their control systems are meeting all of the unilateral constraints required to keep the foot flat on the ground and not slipping.

Section 4 summarizes many of the control approaches being pursued in the field of bipedal robotics. An attempt is made to categorize each control method by the modeling features used in its design. Does it use the full dynamical model or a simplified model? If the latter, what is it? Are hybrid aspects, such as impacts, explicitly taken into account? Are underactuated models allowed? A good sampling of the range of ideas is provided, but the summary is not exhaustive. The large and very interesting literature addressing monopedal robots, polypedal robots, and planar bipedal robots is barely touched upon, and even the presentation of 3D bipedal flat-footed walking using simplified models is not complete. The focus instead in this paper is on control methods that make use of the full-dimensional dynamic models described in Sect. 3 and for which formal statements can be made on the stability properties of solutions of the closed-loop system.

Sections 5-7 summarize two control design methods that are being pursued by subsets of the authors. The primary aim of the work in Sect. 5 is to confront the issue of underactuation. A model is studied where the foot is replaced with a point contact. This can be thought of as walking on stilts or as walking with very small feet so that foot rotation, and hence underactuation, is unavoidable. This study is important precisely because dealing with underactuation has been a stumbling block in the formal development of control laws with provable stability properties for bipedal robots. Section 6 addresses more advanced aspects of the theory presented in Sect. 5; it is much less tutorial in nature.

The primary objective of work presented in Sect. 7 is to develop control techniques which naturally deal with the multi-phase nature of bipedal locomotion, with the early emphasis on gaits which have only fully actuated phases. Extensions to address underactuation are currently being considered and are summarized here. An important idea is that in the fully actuated domains the sagittal and coronal ${ }^{1}$ dynamics of a 3D biped can be decoupled using a variant of geometric reduction termed functional Routhian reduction. It is then only necessary to control the sagittal dynamics of the biped which is achieved through controlled symmetries - this shapes the potential energy of the system to mimic a passive biped walking down a shallow slope. Since both of these control laws require full actuation they are implemented on the fully actuated domains and "local" control laws, motivated by ideas similar to those presented in Sect. 5, are implemented on the underactuated domains to achieve the transitions through these domains.

The paper is concluded in Sect. 8 with a discussion of open problems.

\section{Preliminaries on Hybrid Models, Periodic So- lutions, and Poincaré Maps}

This section overviews two types of hybrid models which occur frequently in models of bipedal locomotion. The dynamic models described in Sect. 3 for bipedal locomotion naturally lead to hybrid systems as given here. The section also introduces two primary tools of stability analysis for periodic orbits in such models, namely the method of Poincaré sections and the notion of a hybrid invariant manifold.

\subsection{Systems with Impulse Effects or Single-Domain Hy- brid Models}

To define a $C^{1}$ system with impulse effects, consider

$$
\dot{x}=f(x),
$$

where the state manifold $\mathcal{X}$ is an open connected subset of $\mathbb{R}^{n}$, and $f$ is a $C^{1}$ vector field on $\mathcal{X}$. A switching surface $\mathcal{S}$ is a co-dimension one $C^{1}$ submanifold with $\mathcal{S}=\left\{x \in \mathcal{X} \mid H(x)=0, \dot{H}(x)=L_{f} H(x)<0\right\}$, where $H: \mathcal{X} \rightarrow \mathbb{R}$ is $C^{1}$ and $\mathcal{S} \neq \emptyset$; because $\forall x \in \mathcal{S}, L_{f} H(x)<$ 0 , it follows that $\frac{\partial H}{\partial x}(x) \neq 0$. A transition or reset $^{2}$ map is a $C^{1}$ function $\Delta: \mathcal{S} \rightarrow \mathcal{X}$, where $\mathcal{S} \cap \Delta(\mathcal{S})=\emptyset$, that is,

\footnotetext{
1 The sagittal plane divides the body into left and right halves; planar robots typically evolve in the sagittal plane. The frontal plane divides the body into front and back halves; hip sway takes place in the frontal plane. The frontal plane is also called the coronal plane.

2 When the reset map corresponds to the swing leg impacting the ground, it is commonly called an impact map instead of a reset map.
} 
the image of the reset map is disjoint from its domain. A $C^{1}$ autonomous system with impulse effects is written as

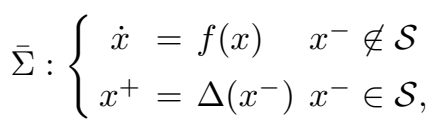

where $x^{-}(t)=\lim _{\tau \nearrow_{t}} x(\tau)$ and $x^{+}(t)=\lim _{\tau \searrow t} x(\tau)$ are the left and right limits of a trajectory, $x(t)$. For compactness of notation, an autonomous system with impulse effects (2) will sometimes be denoted as a 4-tuple, $\bar{\Sigma}=(\mathcal{X}, \mathcal{S}, \Delta, f)$.

In simple terms, a solution of (2) is specified by the differential equation (1) until its state "impacts" the hyper surface $\mathcal{S}$ at some time $t_{I}$. At $t_{I}$, the reset map $\Delta$ compresses the impact event into an instantaneous moment of time, resulting in a discontinuity in the state trajectory. The reset map provides the new initial condition from which the solution of the differential equation continues until the next impact with $\mathcal{S}$. In order to avoid the state having to take on two values at the "impact time" $t_{I}$, the impact event is, roughly speaking, described in terms of the values of the state "just prior to impact" at time " $t_{I}^{-}$", and "just after impact" at time " $t_{I}^{+}$". These values are represented by $x^{-}$and $x^{+}$, respectively. A formal definition of a solution can be written down by piecing together appropriately initialized solutions of (1). A choice must be made whether the solution is a leftor a right-continuous function of time at each impact event; here, solutions are assumed to be right continuous. Other useful notions of a solution can be found in Filippov [1960], Ye et al. [1998], Haddad et al. [2006], Goebel et al. [2009], Lygeros et al. [2003]. Because we are interested in the local stability properties of periodic orbits, we will exclude Zeno and other complex behavior from the systems under study; see Or and Ames [2008, 2009], Lamperski and Ames [2008], Goebel et al. [2009].

\subsection{Periodic Orbits and the Poincaré Return Map for Single-Domain Models}

Cyclic behaviors such as walking are represented as periodic orbits of systems with impulse effects. A solution $\varphi\left(t, t_{0}, x_{0}\right)$ of an autonomous system $\bar{\Sigma}$ is periodic if there exists a finite $T>0$ such that $\varphi\left(t+T, t_{0}, x_{0}\right)=$ $\varphi\left(t, t_{0}, x_{0}\right)$ for all $t \in\left[t_{0}, \infty\right)$. A set $\mathcal{O} \subset \mathcal{X}$ is a periodic orbit if $\mathcal{O}=\left\{\varphi\left(t, t_{0}, x_{0}\right) \mid t \geq t_{0}\right\}$ for some periodic solution $\varphi\left(t, t_{0}, x_{0}\right)$. If a periodic solution has an impact event, then the corresponding periodic orbit $\mathcal{O}$ is not closed; see Grizzle et al. [2001], Morris and Grizzle [2005]. Let $\overline{\mathcal{O}}$ denote its set closure. Notions of stability in the sense of Lyapunov, asymptotic stability, and exponential stability of orbits follow the standard definitions of orbital stability as in [Khalil, 1996, pp. 302], Grizzle et al. [2001], Nersesov et al. [2002].

The method of Poincaré sections is widely used to

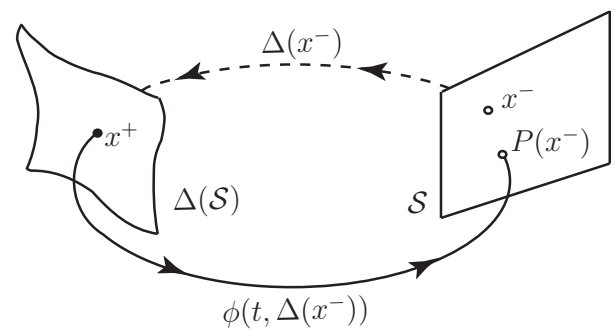

Fig. 1. Geometric interpretation of a Poincaré return map $P: \mathcal{S} \rightarrow \mathcal{S}$ for a system with impulse effects. The Poincaré section is selected as the switching surface, $\mathcal{S}$. A periodic orbit exists when $P\left(x^{-}\right)=x^{-}$. If solutions are assumed to be right continuous, then $x^{-}$is not an element of the orbit; with left-continuous solutions, $\Delta\left(x^{-}\right)$is not an element of the orbit.

determine the existence and stability of periodic orbits in a broad range of system models, such as timeinvariant and periodically-time-varying ordinary differential equations Parker and Chua [1989], Guckenheimer and Holmes [1996], hybrid systems consisting of several time-invariant ordinary differential equations linked by event-based switching mechanisms and re-initialization rules Grizzle et al. [2001], Nersesov et al. [2002], Roup et al. [2003], differential-algebraic equations Hiskens [2001], and relay systems with hysteresis Goncalves et al. [2001], to name just a few. The analytical details may vary significantly from one class of models to another, but, despite these sometimes subtle differences, on a conceptual level the method of Poincaré is consistent and straightforward: sample the solution of a system according to an event-based or time-based rule and then evaluate the stability properties of equilibrium points (also called fixed points) of the sampled system. The sampled values give rise to the Poincaré return map; see Fig. 1. Fixed points of the Poincare map correspond to periodic orbits (limit cycles) of the underlying system.

When using the method of Poincare to study the system[p with impulse effects (2), it is natural to select $\mathcal{S}$ as the Poincaré section. To define the return map, let $\phi\left(t, x_{0}\right)$ be the maximal solution of (1) with initial condition $x_{0}$ at time $t_{0}=0$. The time-to-impact function, $T_{I}: \mathcal{X} \rightarrow \mathbb{R}$, is the time from initialization to the first intersection with the set $\mathcal{S}$ and is in general a partial map [Westervelt et al., 2007a, pp. 90]

$$
\begin{array}{r}
T_{I}\left(x_{0}\right):=\inf \left\{t \geq 0 \mid \phi\left(t, x_{0}\right) \in S\right\} \text { if } \exists t \text { such that } \\
\phi\left(t, x_{0}\right) \in \mathcal{S} .
\end{array}
$$

The Poincaré return map, $P: \mathcal{S} \rightarrow \mathcal{S}$, is then the partial map

$$
P(x)=\phi\left(T_{I} \circ \Delta(x), \Delta(x)\right) .
$$

A periodic orbit $\mathcal{O}$ is period-one if its closure intersects $\mathcal{S}$ at exactly one point, $x^{*}=\overline{\mathcal{O}} \cap \mathcal{S}$; because $x^{*}=P\left(x^{*}\right)$, it is called a fixed point. A period-one orbit is transversal to 
$\mathcal{S}$ if $L_{f} H\left(x^{*}\right)=\frac{\partial H}{\partial x}\left(x^{*}\right) f\left(x^{*}\right) \neq 0$ (in words, the vector field $f$ is not tangent to $\mathcal{S}$ at the point $\left.x^{*}\right)$. For convenience, define the partial function $\phi_{T_{I}}(x)=\phi\left(T_{I}(x), x\right)$ so that the Poincaré return map can be written as

$$
P(x)=\phi_{T_{I}} \circ \Delta(x) .
$$

For the case of autonomous systems with impulse effects, the method of Poincaré sections is formalized in the following theorem.

Theorem 1 (Morris and Grizzle [2009], Method of Poincaré Sections) If the $C^{1}$ autonomous system with impulse effects $\bar{\Sigma}=(\mathcal{X}, \mathcal{S}, \Delta, f)$ has a periodic orbit $\mathcal{O}$ that is transversal to $\mathcal{S}$, then the following are equivalent:

i) $x^{*}$ is an exponentially stable (respectively, asymptotically stable, or stable in the sense of Lyapunov) fixed point of $P$;

ii) $\mathcal{O}$ is an exponentially stable (respectively, asymptotically stable, or stable in the sense of Lyapunov) periodic orbit.

Remark 2 All of the stability notions above are local. Results in [Westervelt et al., 2007a, Sect. 4.2.2] show that if $\mathcal{O}$ is transversal to $\mathcal{S}$ and $\bar{\Sigma}=(\mathcal{X}, \mathcal{S}, \Delta, f)$ is $C^{1}$, then the partial map $P$ is well-defined and differentiable at a fixed point $x^{*}$, and hence exponential stability can be checked by evaluating eigenvalues of the Jacobian of $P$ at $x^{*}$.

\subsection{Multi-domain Hybrid Models}

This section will address systems with $N_{p} \geq 2$ continuous domains and discrete transitions between the domains. Such models frequently occur in bipedal locomotion. We will assume phases are executed in a fixed order $1 \rightarrow 2 \rightarrow \cdots \rightarrow N_{p} \rightarrow 1$. We will use the notation $N_{p}+1=1$ (addition modulo $N_{p}$ ).

For each $1 \leq i \leq N_{p}$, let $\mathcal{X}_{\mathrm{i}}$ be an open connected subset of $\mathbb{R}^{n_{\mathrm{i}}}$ upon which is defined an autonomous differential equation $\mathcal{F}_{\mathrm{i}}$. Let $\mathcal{S}_{\mathrm{i}}^{\mathrm{i}+1}$ be an embedded submanifold of co-dimension one in the state space $\mathcal{X}_{\mathrm{i}}$ that determines when a transition from $\mathcal{X}_{\mathrm{i}}$ to $\mathcal{X}_{\mathrm{i}+1}$ takes place according to the reset map $\Delta_{\mathrm{i}}^{\mathrm{i}+1}: \mathcal{S}_{\mathrm{i}}^{\mathrm{i}+1} \rightarrow \mathcal{X}_{\mathrm{i}+1}$.

The corresponding hybrid model is written using the no- tation in Guckenheimer and Johnson [1995] as

$$
\bar{\Sigma}:\left\{\begin{array}{c}
\mathcal{X}=\left\{\mathcal{X}_{\mathrm{i}}\right\}_{\mathrm{i}=1}^{N_{p}}: \mathcal{X}_{\mathrm{i}} \subset \mathbb{R}^{n_{\mathrm{i}}} \\
\mathcal{F}=\left\{f_{\mathrm{i}}\right\}_{\mathrm{i}=1}^{N_{p}}: \dot{x}_{\mathrm{i}}=f_{\mathrm{i}}\left(x_{\mathrm{i}}\right) \\
\mathcal{S}=\left\{\mathcal{S}_{\mathrm{i}}^{\mathrm{i}+1}\right\}_{\mathrm{i}=1}^{N_{p}}: \mathcal{S}_{\mathrm{i}}^{\mathrm{i}+1}=\left\{x_{\mathrm{i}} \in \mathcal{X}_{\mathrm{i}} \mid H_{\mathrm{i}}^{\mathrm{i}+1}\left(x_{\mathrm{i}}\right)=0,\right. \\
\left.\Delta=\left\{\Delta_{\mathrm{i}}^{\mathrm{i}+1}\right\}_{\mathrm{i}=1}^{N_{p}}: x_{\mathrm{i}+1}^{+\mathrm{i}+1}\left(x_{\mathrm{i}}\right)<0\right\} \\
\Delta \Delta_{\mathrm{i}}^{\mathrm{i}+1}\left(x_{\mathrm{i}}^{-}\right) .
\end{array}\right.
$$

Under assumptions analogous to those for the singledomain model, a unique, maximal solution of the multidomain model can be constructed by piecing together trajectories of the flows $\mathcal{F}_{\mathrm{i}}$ in such a way that a transition occurs when a flow intersects a switching hyper-surface, $\mathcal{S}_{\mathrm{i}}^{\mathrm{i}+1}$, and at each transition, the new initial condition is determined by the reset maps $\Delta_{i}^{i+1}$. To avoid chattering, it is assumed that $\Delta_{i}^{i+1}\left(\mathcal{S}_{i}^{i+1}\right) \cap \mathcal{S}_{i+1}^{i+2}=\emptyset$, so that a solution through a domain must have a non-zero duration.

Remark 3 Note that the hybrid model $\bar{\Sigma}$ introduced in (4) is equivalent to the definition of a hybrid system on a cycle (as studied in Lamperski and Ames [2008]), which is typically stated as a tuple,

$$
\mathscr{H}=\bar{\Sigma}=(\Gamma, \mathcal{X}, \mathcal{S}, \Delta, \mathcal{F}),
$$

where $\Gamma$ is the directed graph with vertices $\left\{1,2, \ldots, N_{p}\right\}$ and edges connecting vertex $i$ to vertex $i+1$.

\subsection{Periodic Orbits and the Poincaré Return Map for Multi-Domain Models}

Let $\mathcal{X}=\mathcal{X}_{1} \cup \mathcal{X}_{2} \cdots \cup \mathcal{X}_{N_{p}}$. A solution $\phi(t)$ of (4) is periodic if there exists a finite $T>0$ such that $\phi(t+T)=\phi(t)$ for all $t \in\left[t_{0}, \infty\right)$. A set $\mathcal{O} \subset \mathcal{X}$ is a periodic orbit of (4) if $\mathcal{O}=\left\{\phi(t) \mid t \geq t_{0}\right\}$ for some periodic solution $\phi(t)$. The definitions of orbital stability in the sense of Lyapunov, orbital asymptotic stability, and orbital exponential stability are analogous to those for systems with impulse effects. A periodic orbit $\mathcal{O}$ is transversal to $\mathcal{S}_{i}^{\mathrm{i}+1}$ if its closure intersects $\mathcal{S}_{\mathrm{i}}^{\mathrm{i}+1}$ in exactly one point, and for $x_{\mathrm{i}}^{*}:=\overline{\mathcal{O}} \cap \mathcal{S}_{\mathrm{i}}^{\mathrm{i}+1}, L_{f_{\mathrm{i}}} H_{\mathrm{i}}^{\mathrm{i}+1}\left(x_{\mathrm{i}}^{*}\right):=\frac{\partial H_{\mathrm{i}}^{\mathrm{i}+1}}{\partial x_{\mathrm{i}}}\left(x_{\mathrm{i}}^{*}\right) f_{\mathrm{i}}\left(x_{\mathrm{i}}^{*}\right) \neq 0$. A periodic orbit $\mathcal{O}$ is transversal if it is transversal to $\mathcal{S}_{i}^{\mathrm{i}+1}$ for all $i$. In the case of a bipedal robot, a nontrivial, transversal, periodic orbit will also be referred to as periodic locomotion.

The Poincaré return map remains the mathematical tool of choice for determining the existence and stability properties of periodic orbits. As in (3), define the phase- $i$ time-to-impact function, $T_{I, \mathrm{i}}: \mathcal{X}_{\mathrm{i}} \rightarrow \mathbb{R}$ as the 
partial map

$$
\begin{array}{r}
T_{I, \mathrm{i}}\left(x_{0}\right):=\inf \left\{t \geq 0 \mid \phi_{\mathrm{i}}\left(t, x_{0}\right) \in \mathcal{S}_{\mathrm{i}}^{\mathrm{i}+1}\right\} \text { if } \exists t \text { such that } \\
\phi_{\mathrm{i}}\left(t, x_{0}\right) \in \mathcal{S}_{\mathrm{i}}^{\mathrm{i}+1},
\end{array}
$$

where $\phi_{\mathrm{i}}\left(t, x_{0}\right)$ is an integral curve of (4) corresponding to $\phi_{\mathrm{i}}\left(0, x_{0}\right)=x_{0}$. The generalized Poincaré phase- $i$ map $P_{\mathrm{i}}: \mathcal{S}_{\mathrm{i}-1}^{\mathrm{i}} \rightarrow \mathcal{S}_{\mathrm{i}}^{\mathrm{i}+1}$ is the partial map

$$
P_{\mathrm{i}}\left(x_{\mathrm{i}-1}\right):=\phi_{\mathrm{i}}\left(T_{I, \mathrm{i}}\left(\Delta_{\mathrm{i}-1}^{\mathrm{i}}\left(x_{\mathrm{i}-1}\right)\right), \Delta_{\mathrm{i}-1}^{\mathrm{i}}\left(x_{\mathrm{i}-1}\right)\right) .
$$

The Poincaré return map can be defined as the composition of the generalized Poincaré phase- $i$ maps, starting at any point in the cycle $1 \rightarrow 2 \rightarrow \cdots \rightarrow N_{p} \rightarrow 1$. Here, for convenience, we start it at $i=1$, so that

$$
P:=P_{N_{p}} \circ \cdots \circ P_{1}
$$

Proposition 4 (Connecting Multi-Phase Models to Single-Phase Models) Let $P$ be the Poincaré return map defined in (7) for the multi-phase model in (4). $P$ is also the Poincaré return map for the system with impulse effects (2), where $\mathcal{X}=\mathcal{X}_{1}, f=f_{1}, \mathcal{S}:=\mathcal{S}_{1}^{2}$ and $\Delta:=$ $\Delta_{N_{p}}^{1} \circ P_{N_{p}} \circ \cdots \circ P_{2}$.

PROOF. This follows immediately from the construction of the Poincaré return maps in (4) and (7).

Remark 5 It is emphasized that this observation is important because it allows results developed for singledomain models of the form (2) to be applied to models with multiple phases, as in (4). In particular, suppose that the multi-domain hybrid model (4) is $C^{1}$ in each phase and has a transversal periodic orbit $\mathcal{O}$. Then, results in [Westervelt et al., 2007a, Sect. 4.2.2/ show that $\Delta:=\Delta_{N_{p}}^{1} \circ P_{N_{p}} \circ \cdots \circ P_{2}$ is $C^{1}$ in a neighborhood of $x^{*}=\mathcal{O} \cap \mathcal{S}$, and thus $P$ is $C^{1}$ in a neighborhood of $x^{*}$. Exponential stability can therefore be checked by evaluating eigenvalues of the Jacobian of $P$ at $x^{*}$.

\subsection{Determining Orbital Stability on the Basis of a Re- striction Dynamics}

This section identifies properties of the autonomous hybrid system (2) under which the exponential stability of a periodic orbit can be determined on the basis of a hybrid restriction dynamics. The key hypothesis will be the existence of an embedded submanifold that is invariant under both the continuous and discrete portions of the hybrid model (2). The design of static and dynamic state variable feedbacks that create invariant submanifolds for systems modeled by ordinary differential equations is a well-studied problem and plays a prominent role in the notion of the zero dynamics. How to design feedbacks that achieve invariance under the reset map is treated in Sect. 5.3.

The following definitions formalize notions of hybrid invariance and restriction dynamics.

Definition 6 For an autonomous system with impulse effects $\bar{\Sigma}=(\mathcal{X}, \mathcal{S}, \Delta, f)$, a submanifold $Z \subset \mathcal{X}$ is forward invariant if for each point $x$ in $\mathcal{Z}, f(x) \in T_{x} Z$ where $T_{x} Z$ is the tangent space of the manifold $Z$ at the point $x$. A submanifold $Z$ is impact invariant in an autonomous system with impulse effects $\bar{\Sigma}$, if for each point $x$ in $\mathcal{S} \cap Z$, $\Delta(x) \in Z$. A submanifold $Z$ is hybrid invariant if it is both forward invariant and impact invariant.

It follows that if $Z$ is hybrid invariant, solutions that start in $Z$, remain in $Z$, even after a switching (or reset) event.

Definition 7 If a $C^{1}$ embedded submanifold $Z$ is hybrid invariant and $\mathcal{S} \cap Z$ is $C^{1}$ with dimension one less than that of $Z$, then

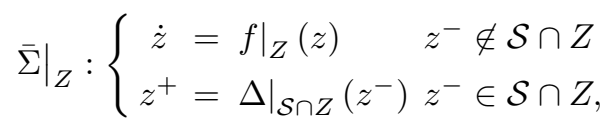

is called a hybrid restriction dynamics of the autonomous system $\bar{\Sigma}$, where $\left.f\right|_{Z}$ and $\left.\Delta\right|_{\mathcal{S} \cap Z}$ are the restrictions of $f$ and $\Delta$ to $Z$ and $\mathcal{S} \cap Z$, respectively. The hybrid restriction system (8) is denoted as

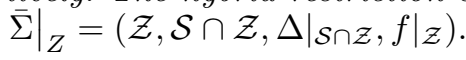

If a system $\bar{\Sigma}$ has a periodic orbit $\mathcal{O}$ lying in a hybrid invariant manifold $Z$, then $\mathcal{O}$ is a periodic orbit of the resulting hybrid restriction dynamics. In this case hybrid invariance of $\mathcal{Z}$ is reflected in the Poincaré map as

$$
P(\mathcal{S} \cap Z) \subset \mathcal{S} \cap Z .
$$

On the basis of (9), the restricted Poincaré map,

$$
\rho: \mathcal{S} \cap Z \rightarrow \mathcal{S} \cap Z,
$$

is defined as $\rho=\left.P\right|_{Z}$.

The following result shows that if the invariant manifold is sufficiently rapidly attractive, then stability of the periodic orbit in the complete model can be deduced on the basis of the restricted Poincaré map.

Theorem 8 (Morris and Grizzle [2009], Reduced Dimensional Stability Test) Consider a family of $C^{1}$ autonomous systems with impulse effects with the vector field of each member depending on a real parameter $\epsilon>0, \bar{\Sigma}^{\epsilon}=\left(\mathcal{X}, \mathcal{S}, \Delta, f^{\epsilon}\right)$. Let $Z$ be a $k$-dimensional $C^{1}$ embedded submanifold of $\mathcal{X}$, with $k \geq 1$. Suppose in addition that: 
(a) $\mathcal{S} \cap Z$ is a $C^{1},(k-1)$-dimensional embedded submanifold of $\mathcal{S}$;

(b) $f^{\epsilon}$ restricted to $\mathcal{Z}$ is independent of $\epsilon$, so that $\left.f\right|_{Z}=$ $\left.{ }^{\epsilon}\right|_{Z}$ for any $\epsilon \in(0, \infty)$;

(c) $Z$ is hybrid invariant;

(d) there exists a periodic orbit $\mathcal{O}$ of $\bar{\Sigma}^{\epsilon}=\left(\mathcal{X}, \mathcal{S}, \Delta, f^{\epsilon}\right)$ that is contained in $Z$ and with the corresponding fixed-point denoted by $x^{*}$; and

(e) there exists a function $K:(0, \infty) \rightarrow[0, \infty)$ such that $\lim _{\epsilon \backslash 0} K(\epsilon)=0$, and $\forall \epsilon>0, \exists \delta>0$ such that ${ }^{3} \forall x_{0} \in B_{\delta}\left(\Delta\left(x^{*}\right)\right)$, $\operatorname{dist}\left(\phi_{T_{I}}^{\epsilon}\left(x_{0}\right), Z\right) \leq$ $K(\epsilon) \operatorname{dist}\left(x_{0}, Z\right)$.

Then the restriction dynamics $\left.\bar{\Sigma}^{\epsilon}\right|_{Z}$ and fixed point are both independent of $\epsilon$. In addition, there exists $\bar{\epsilon}>0$ such that for $0<\epsilon<\bar{\epsilon}$, the following are equivalent:

i) $x^{*}$ is an exponentially stable fixed point of $P^{\epsilon}$, and ii) $x^{*}$ is an exponentially stable fixed point of $\rho$,

where $P^{\epsilon}=\phi_{T_{I}}^{\epsilon} \circ \Delta$ and $\rho=\left.P^{\epsilon}\right|_{\mathcal{Z}}$.

\section{Dynamic Models}

Robotic legged locomotion is characterized by the fact that the contact between the robot and its environment (the ground) is unilateral and intermittent. Unilateral constraints entail "complementarity conditions", that is, conditions requiring that the product of two nonnegative quantities be zero. For example, when the distance from a contact point to the ground is greater than zero (i.e., non-contact), the ground cannot exert a force (i.e., force must be zero). The distance cannot be negative since the foot cannot penetrate the ground (ground and foot are modeled as rigid bodies). When the foot is in contact with the ground (i.e., distance is zero), then the ground reaction force must be positive or zero since the ground can push, but cannot pull, on the robot. Additional constraints to avoid (or to allow) slipping also exist.

While the dynamic models developed in Moreau [1966] and Hürmüzlü et al. [2004] on the basis of complementarity conditions are very general, effective means to design controllers for models of this form are not known at this time. For the purposes of control design, an actuated version of the multi-phase hybrid models in (4) are used, where each phase corresponds to a specific combination of complementarity conditions being either zero or nonzero. For periodic walking, the sequence of complementarity conditions gives rise to distinct phases, each corresponding to different modeling constraints. Enumerating these constraints leads to a natural way of struc-

\footnotetext{
$\overline{3} B_{r}(x)$ denoted the open ball of radius $r$ about the point $x$.
}

turing a walking gait as a directed graph, thereby motivating the application of hybrid systems theory.

In the following, Sect. 3.1 presents a biped model in terms of complementarity conditions. The detailed calculation of the dynamic models for different phases of a walking gait are developed systematically in Sect. 3.2 to 3.6. The overall hybrid model of a walking gait is assembled in Sect. 3.7.

\subsection{Generalities}

The robot itself is classically modeled as a tree structure composed of rigid links. When contact occurs between the feet and the ground, it is assumed to be a rigid contact. With these assumptions, one way to obtain a model for the various phases of a walking gait is to first construct a floating-base Lagrangian model of the robot (i.e., no assumptions on ground contact), and then add in ground contact forces via D'Alembert's principle.

To begin this modeling approach, let $R_{0}$ be a fixed inertial (or world) frame and let $R_{b}$ be a reference frame attached to some point on the robot, as in Fig. 2. Let $p_{b} \in \mathbb{R}^{3}$ be the Cartesian position of $R_{b}$ with respect to $R_{0}$ and let $\phi_{b} \in S O(3)$ be the orientation. Where convenient, we identify an open subset of $S O(3)$ with an open subset of $\mathbb{R}^{3}$ using Euler angles ${ }^{4}$. Next, let $q \in \mathcal{Q}$ be an $N$-dimensional vector of body (or shape) coordinates ${ }^{5}$ for the robot. Then $q_{e}=\left(p_{b}^{\prime}, \phi_{b}^{\prime}, q\right)^{\prime} \in \mathcal{Q}_{e}=$ $\mathbb{R}^{3} \times S O(3) \times Q$ is a set of generalized coordinates for the robot.

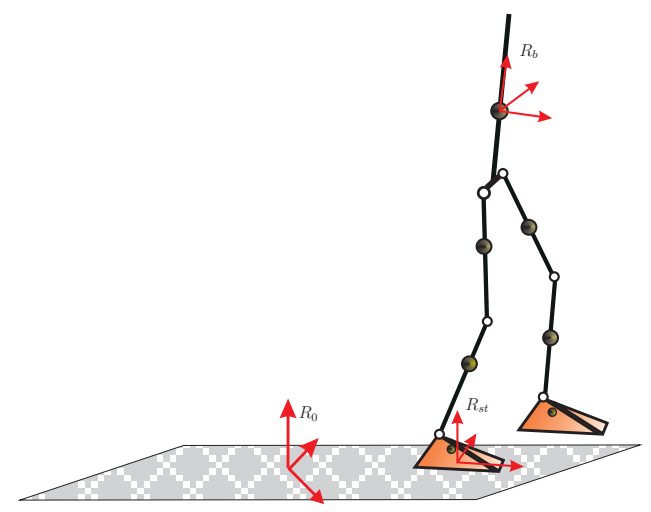

Fig. 2. A frame $R_{b}$ is attached to the body. The position and orientation of the robot are expressed with respect to a fixed inertial frame $R_{0}$. A frame $R_{s t}$ attached to the stance foot is useful for expressing the contact conditions.

\footnotetext{
4 While singular configurations exist in this identification, they are not relevant for upright configurations of the torso that occur in normal walking.

5 These are coordinates tied to the reference frame $R_{b}$ on the body of the robot. If the robot consists of rigid links connected through one-dimensional revolute joints, then the relative joint angles constitute a set of body coordinates.
} 
Following standard techniques, the robot's Lagrangian is computed as a functional acting on $T \mathcal{Q}_{e}$. The Lagrangian is defined to be the difference between the kinetic and potential energies

$$
\mathcal{L}_{e}\left(q_{e}, \dot{q}_{e}\right):=K_{e}\left(q_{e}, \dot{q}_{e}\right)-V_{e}\left(q_{e}\right) .
$$

From Hamilton's principle, the equations of motion can be calculated directly from the Lagrangian as

$$
\frac{d}{d t} \frac{\partial \mathcal{L}_{e}}{\partial \dot{q}_{e}}-\frac{\partial \mathcal{L}_{e}}{\partial q_{e}}=\Gamma\left(q_{e}, \dot{q}_{e}\right)+B_{e}\left(q_{e}\right) u,
$$

where $B_{e}(q) u$ is the vector of actuator torques and $\Gamma\left(q_{e}, \dot{q}_{e}\right)$ represents other nonconservative forces (such as joint friction) [Goldstein et al., 2002, pp. 34-45]. The effect of gravity is accounted for in the potential energy. The torque distribution matrix $B_{e}(q)$ depends only on the body coordinates; its columns are assumed involutive and (point-wise) linearly independent so that in appropriate coordinates the matrix is constant, with rank equal to the number of actuators, $N_{a}$. The kinetic energy is a quadratic, positive definite function of the generalized velocities, and hence (12) leads to the standard robot equations

$$
D_{e}\left(q_{e}\right) \ddot{q}_{e}+C_{e}\left(q_{e}, \dot{q}_{e}\right) \dot{q}_{e}+G_{e}\left(q_{e}\right)-\Gamma\left(q_{e}, \dot{q}_{e}\right)=B_{e}(q) u .
$$

From here on out, for simplicity, the term $\Gamma\left(q_{e}, \dot{q}_{e}\right)$ will be dropped; it is very easy to add it back in when required. $D_{e}\left(q_{e}\right)$ is the $(N+6) \times(N+6)$ inertia matrix, $C_{e}\left(q_{e}, \dot{q}_{e}\right) \dot{q}_{e}$ is the vector of Coriolis and centrifugal effects, $G_{e}\left(q_{e}\right)$ is the gravitational force, $u$ is the vector of inputs (i.e, the actuator torques). Sometimes, to simplify notation, the Coriolis, centrifugal and gravity terms are grouped into a single vector $H_{e}\left(q_{e}, \dot{q}_{e}\right)=C_{e}\left(q_{e}, \dot{q}_{e}\right) \dot{q}_{e}+G_{e}\left(q_{e}\right)$.

At this point, the model (13) assumes no contact with the ground. A general form of the dynamic model of the biped with $m$ points belonging to the feet that can be in contact with the environment (for example the 8 corners of the two feet), is called a complementarity dynamical system in Hürmüzlü et al. [2004]. It can be written as

$$
\begin{gathered}
D_{e}\left(q_{e}\right) \ddot{q}_{e}+H_{e}\left(q_{e}, \dot{q}_{e}\right)=B_{e}(q) u+\nabla F\left(q_{e}\right) \lambda_{n}+P_{t}\left(q_{e}, \dot{q}_{e}\right) \\
\lambda_{n}^{T} F\left(q_{e}\right)=0 ; \lambda_{n} \geq 0 ; F\left(q_{e}\right) \geq 0
\end{gathered}
$$

where $F\left(q_{e}\right)$ is a vector composed of the $m$ vertical distances between the possible points of contact of the feet and the ground; $\lambda_{n}$ is a vector composed of the $m$ normal forces exerted by the ground on these points of contact; and $P_{t}\left(q_{e}, \dot{q}_{e}\right)$ captures the tangential forces exerted by the ground. This latter term depends on the model of friction (16) and while the consideration of both sliding and sticking phenomena can be expressed through complementarity conditions, these are not detailed here.
The orthogonality condition $\lambda_{n}^{T} F\left(q_{e}\right)=0$ means that for each point $1 \leq i \leq m$, if $F\left(q_{e}\right)(i)>0$, i.e., there is no contact, then the normal force is $\lambda_{n}(i)=0$, whereas a non-zero but positive contact force $\lambda_{n}(i)>0$ is possible only if there is contact, i.e., $F\left(q_{e}\right)(i)=0$. The model (14) clearly shows the possible intermittence of the contact. When a contact is lost, the corresponding contact force switches from a positive to a zero value and this is taken into the model without problem. When a contact occurs, $F\left(q_{e}\right)(i)$ decreases to zero and cannot be negative. If the derivative $\dot{F}\left(q_{e}\right)(i)$ is different from zero when $F\left(q_{e}\right)(i)=0$, then a discontinuity will appear and it must be treated with a restitution law, as in (17).

Complementarity Lagrangian systems as in (14)-(17) were first introduced by Moreau [1966]. Models of this form are very useful for simulating bipedal robot locomotion because of the possibility to detect changes in ground contact, even unexpected changes such as foot scuffing or a foot rolling on an edge. In Posa et al. [2014], complementarity Lagrangian models have been used for trajectory optimization, without a priori enumeration of the type and order of the contact events. In the context of control law design, however, to the best of our knowledge, the first step is to define the sequence and type of contact with the environment, leading to hybrid models of the form used in Sect. 2.3 and illustrated later in Fig. 12. Defining the contact sequence means defining for each phase of a gait whether $\lambda_{n}(i)=0$ or $F\left(q_{e}\right)(i)=0$. Thus, for control law design, the general form (14)-(17) is primarily useful as a conceptual tool. Complementarity models highlight the importance of checking the positivity of the normal force when a contact is assumed. In the development of a control law, preference is given to the definition of specialized models that include a minimal set of state variables.

In following sections we will develop models for three types of contact, namely, the stance foot is flat on the ground, the foot is rotating about an axis corresponding to the heel or the toe, and the limiting case of a stance foot reduced to a point contact. The term single support (SS) means that one foot is in contact with the ground, flat or otherwise, and double support (DS) means that both feet are in contact with the ground. The same terminology is used when a leg is terminated in a point instead of a foot.

\subsection{Single support with flat foot contact}

Assuming at least three non-collinear points of contact, the net effect of the ground reaction forces can be summarized as a reaction wrench expressed in a frame attached to the foot. Let $R_{s t}$ be a reference frame attached to the stance foot as in Fig. 2. The ground contact wrench 
$F_{s t}$ has six components ${ }^{6}$,

$$
F_{s t}=\left(F_{s t}^{f x}, F_{s t}^{f y}, F_{s t}^{f z}, F_{s t}^{m x}, F_{s t}^{m y}, F_{s t}^{m z}\right)^{\prime},
$$

where the first three components are the ground reaction forces and the last three are the moments (i.e., ground reaction torques). Because $F_{s t}^{f z}$ is a normal force, it is unilateral. If the reaction wrench is calculated in a different point (not at the origin of frame $R_{s t}$ ), the values of the moments change. It can be shown that for planar horizontal ground, there exists a point on the ground where the moments around the horizontal axes $F_{s t}^{m x}, F_{s t}^{m y}$ are zero; this point is called the Center of pressure $(\mathrm{CoP})$ or Zero Moment Point (ZMP). It is known that flat-footed walking requires this point to be in the interior of the convex hull of the foot Vukobratović and Borovac [2004].

The position and orientation of the frame $R_{s t}$ in the inertial frame $R_{0}$ can be expressed by a $(4 \times 4)$ transformation matrix denoted $T_{0}^{s t}$, which is a function of $q_{e}$. The linear and angular velocities of the foot, $v_{s t}$ and $\omega_{s t}$, are then defined by

$$
\left[\begin{array}{cccc}
0 & -\omega_{s t}^{z} & \omega_{s t}^{y} & v_{s t}^{x} \\
\omega_{s t}^{z} & 0 & -\omega_{s t}^{x} & v_{s t}^{y} \\
-\omega_{s t}^{y} & \omega_{s t}^{x} & 0 & v_{s t}^{z} \\
0 & 0 & 0 & 0
\end{array}\right]=\left(T_{0}^{s t}\left(q_{e}\right)\right)^{-1} \dot{T}_{0}^{s t}\left(q_{e}, \dot{q}_{e}\right)
$$

According to the principle of virtual work found in Dombre and Khalil [2002], Murray et al. [1993], Spong et al. [2005], the contact wrench is taken into account in the dynamic model as

$$
D_{e}\left(q_{e}\right) \ddot{q}_{e}+H_{e}\left(q_{e}, \dot{q}_{e}\right)=B_{e}(q) u+J_{s t}\left(q_{e}\right)^{\prime} F_{s t},
$$

where $J_{s t}\left(q_{e}\right)$ is full rank and satisfies

$$
\left[\begin{array}{c}
v_{s t} \\
\omega_{s t}
\end{array}\right]=J_{s t}\left(q_{e}\right) \dot{q}_{e} .
$$

This model is similar to (14), but adapted to the case of single support on a flat foot (i.e., the stance foot), with the other foot raised above the ground (i.e., the swing foot).

The position and orientation of the stance foot is assumed to be fixed (no slipping, etc.), and the distance

\footnotetext{
6 In the complementarity representation, a 3-dimensional ground reaction force would be attached to each of the $m \geq 3$ contacts, using the normal forces $\lambda_{n}$ and the tangential forces captured in $P_{t}$. The moments would be generated by the forces acting at a distance from the origin of $R_{s t}$.
}

between each of the points of contact of the stance foot and the ground is assumed to be zero. We will impose these conditions with a holonomic constraint. Let the position and orientation ${ }^{7}$ of $R_{s t}$ with respect to $R_{0}$ (see Fig. 2), expressed in terms of the generalized coordinates $q_{e}$, be denoted by

$$
\eta_{s t}\left(q_{e}\right)=\left[\begin{array}{l}
p_{s t}\left(q_{e}\right) \\
\phi_{s t}\left(q_{e}\right)
\end{array}\right] .
$$

It can be shown that the Jacobian $\frac{\partial \eta_{s t}}{\partial q_{e}}$ has full rank. We can write a holonomic constraint as

$$
\eta_{s t}\left(q_{e}\right)=\left[\begin{array}{l}
p_{s t} \\
\phi_{s t}
\end{array}\right]=\text { constant. }
$$

Because the position and orientation of the foot are fixed, their velocity and acceleration are zero. The kinematic constraint is

$$
\left[\begin{array}{c}
v_{s t} \\
\omega_{s t}
\end{array}\right]=J_{s t}\left(q_{e}\right) \dot{q}_{e}=0_{6 \times 1},
$$

where the velocities are defined in (18). Differentiating (23), the constraint on acceleration is

$$
J_{s t}\left(q_{e}\right) \ddot{q}_{e}+\frac{\partial}{\partial q_{e}}\left(\frac{\partial J_{s t}\left(q_{e}\right)}{\partial q_{e}} \dot{q}_{e}\right) \dot{q}_{e}=0_{6 \times 1} .
$$

This equation in conjunction with (19) leads to a system of equations from which the contact wrench $F_{s t}\left(q_{e}, \dot{q}_{e}, u\right)$ and $\ddot{q}_{e}$ can be computed. It follows that $F_{s t}$ is affine in the actuator torques $u$.

The foot remaining fixed and flat on the ground imposes specific bounds on $F_{s t}$. In order to avoid the foot lifting from the ground, the constraint is

$$
F_{s t}^{f z}>0 .
$$

Since we represent (dry) friction by a classical Amontons-Coulomb model, to avoid linear slipping ${ }^{8}$, the constraint is

$$
\sqrt{\left(F_{s t}^{f x}\right)^{2}+\left(F_{s t}^{f y}\right)^{2}}<\mu F_{s t}^{f z},
$$

where $\mu$ is the assumed friction parameter. The parameter $\mu$ does not directly affect the model (19) because

\footnotetext{
7 Euler angles can be used for example to express the orientation.

8 A condition also exists also on $F_{s t}^{m z}$, but it involves the size of the foot, the friction parameter and the unknown distribution of the forces along the sole of the foot. Consequently, when modeling straight displacement, a constraint on $F_{s t}^{m z}$ in order to avoid rotational slipping is usually neglected.
} 


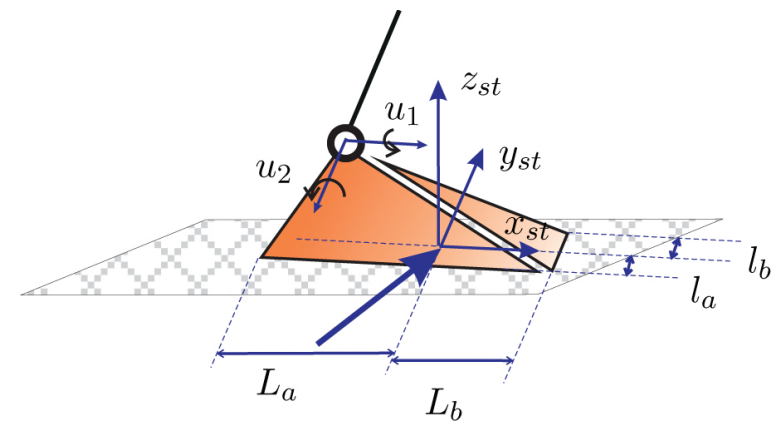

Fig. 3. The ground exerts a wrench on the foot, that is, a pure force and a moment (or torque). The wrench is expressed in the reference frame $R_{s t}$, which has been attached to an arbitrary point on the foot.

the foot is assumed to be not slipping, however it directly affects the domain of validity of the model; as a consequence, the results obtained are not sensitive to the choice of $\mu$ when the constraints are far from their limits ${ }^{9}$. To use linear constraints, the friction cone (26) can be replaced by a friction pyramid, which gives

$$
\begin{aligned}
& \left|F_{s t}^{f x}\right|<\frac{\mu}{\sqrt{2}} F_{s t}^{f z}, \\
& \left|F_{s t}^{f y}\right|<\frac{\mu}{\sqrt{2}} F_{s t}^{f z} .
\end{aligned}
$$

Even if the foot is pressing against the ground and not sliding, it could be in rotation about one of its edges. Indeed, due to the finite size of the feet, and due to the unilateral nature of the contact, Vukobratović et al. [1990] and Chevallereau et al. [2009a] show that the moment produced by the ground is limited by

$$
\begin{gathered}
-l_{b} F_{s t}^{f z}<F_{s t}^{m x}<l_{a} F_{s t}^{f z} \\
-L_{a} F_{s t}^{f z}<F_{s t}^{m y}<L_{b} F_{s t}^{f z},
\end{gathered}
$$

where $L_{a}, L_{b}, l_{a}, l_{b}$ are defined by the geometry of the foot as in Fig. 3. If one of the conditions in (28) is not satisfied, the foot will rotate along an edge. The constraint (28) is known as the Zero Moment Point (ZMP) condition. Because a foot is typically less wide than long, the constraints in the frontal plane are more severe than in the sagittal plane.

The collection of constraints (25), (27), and (28) must all be satisfied for the assumption of walking with a foot flat on the ground to be correct. These equations can be grouped as

$$
A_{F_{s t}}\left(q_{e}\right)^{\prime} F_{s t}\left(q_{e}, \dot{q}_{e}, u\right)>0,
$$

where it is noted that $F_{s t}$ depends not only on the states $q, \dot{q}$, but also on the actuator torques, $u$. The dynamic

\footnotetext{
9 Even if a sliding phase can be useful, we limit our presen-
} tation to walking without sliding. model is valid only if $F_{s t}$ satisfies the condition (29); otherwise, the assumed contact conditions are not valid and the phase of walking changes.

The phase of a walking gait can also change due to other conditions, for example the distance of the swing foot to the ground becoming zero. We introduce therefore a unilateral constraint, $h: \mathcal{Q}_{e} \rightarrow \mathbb{R}$, which parameterizes distance to impact. We can combine unilateral and holonomic constraints viz.

$$
H\left(q_{e}, \dot{q}_{e}, u\right)=\left[\begin{array}{c}
A_{F_{s t}}\left(q_{e}\right)^{\prime} F_{s t}\left(q_{e}, \dot{q}_{e}, u\right) \\
h\left(q_{e}\right)
\end{array}\right]
$$

The domain of admissibility corresponds to the subset in which all of the previous constraints, both holonomic and unilateral, are satisfied,

$$
\mathcal{D}=\left\{\left(\begin{array}{c}
q_{e} \\
\dot{q}_{e} \\
u
\end{array}\right) \in T \mathcal{Q}_{e} \times \mathbb{R}^{N_{a}} \mid H\left(q_{e}, \dot{q}_{e}, u\right)>0\right\}
$$

If the state of the robot belongs to the domain of admissibility, then a simplified model can be used, corresponding to restricting the dynamics to the surface defined by the holonomic constraint (22). The dynamic model then takes the form ${ }^{10}$

$$
D(q) \ddot{q}+C(q, \dot{q}) \dot{q}+G(q)=B(q) u
$$

If each of the joints of the robot, including the ankle, is independently actuated, then the model is fully actuated, meaning $\operatorname{dim} q=\operatorname{dim} u=\operatorname{rank}$ of $B$; otherwise, it is underactuated.

Remark 9 When employing the reduced model (32), the condition of belonging to the domain of admissibility must still be checked. This step is neglected in many publications. As a result, one does not know if the closed-loop model is implicitly assuming a foot of infinite size (so that (28) is hard to violate), a "sticky" ground model, where (25) is violated, or an unrealistically large coefficient of friction in (27). Along a trajectory of (32), the holonomic constraint (22) allows the "missing" components of $q_{e}$ and $\dot{q}_{e}$ to be computed, which in conjunction with the control input used in (32), allows the contact wrench to be evaluated.

Remark 10 The constraint (28) is the most difficult to meet and hence many control strategies are devoted to its satisfaction; see Hirai et al. [1998], Kajita et al. [2003] and references therein. The difficulty in satisfying this

${ }^{10} \mathrm{D}$ is positive definite and the columns of $B$ remain involutive and linearly independent. 
constraint increases as the size of the feet $\left(L_{a}, L_{b}, l_{a}, l_{b}\right)$ decreases. In Sect. 3.4, we will study the situation where all of theses values are equal to zero, corresponding to a point-foot model. A control strategy which can deal with this case should be extendable to a foot of any size.

Remark 11 Section 7 considers a model where the stance knee angle $q_{s k}$ is mechanically locked. In this case, a holonomic constraint $\eta_{s k}=q_{s k}-q_{s k}^{l}$ must be appended to the constraint $\eta$ in (21) and (22). The Jacobian $J_{\text {st }}$ is thus augmented by an additional line $\frac{\partial \eta_{s k}\left(q_{e}\right)}{\partial q_{e}}$. Correspondingly, the reaction $F_{\text {st }}$ is completed by the torque applied by the mechanical locking system, and the torque applied by the knee actuator can be set to zero. If the mechanical looking system is unilateral, a unilateral constraint on the knee torque must be added.

\subsection{Rotation of the foot about the toe or the heel}

A situation where the foot is not flat on the ground is analyzed next. It is assumed that the foot is in rotation along an axis aligned with the toe or the heel and that the $y$-axis of the reference frame $R_{s t}$ has been aligned with the axis of rotation of the foot. It is assumed also that the orientation of the frame $R_{s t}$ is defined by a set of three rotations, with the third rotation being along the $y$-axis, so that the orientation matrix between $R_{0}$ and $R_{s t}$ can be written as ${ }^{0} A_{s t}=\operatorname{Rot}\left(z, \phi_{s t}^{z}\right) \operatorname{Rot}\left(x, \phi_{s t}^{x}\right) \operatorname{Rot}\left(y, \phi_{s t}^{y}\right)$. It follows that the angle $\phi_{s t}^{y}$ is free, while the variables $\phi_{s t}^{x}$ and $\phi_{s t}^{z}$ are constant. Let

$$
\eta_{s t, R}\left(q_{e}\right)=\left[\begin{array}{c}
p_{s t}\left(q_{e}\right) \\
\phi_{s t}^{x}\left(q_{e}\right) \\
\phi_{s t}^{z}\left(q_{e}\right)
\end{array}\right]
$$

be the position of $R_{s t}$ with respect to $R_{0}$ and its orientation about the $x$ and $z$ axes, expressed in terms of the generalized coordinates $q_{e}$. It can be shown that the Jacobian $\frac{\partial \eta_{s t, R}}{\partial q_{e}}$ has full rank. The corresponding holonomic constraint is

$$
\eta_{s t, R}\left(q_{e}\right)=\left[\begin{array}{c}
p_{s t} \\
\phi_{s t}^{x} \\
\phi_{s t}^{z}
\end{array}\right]=\text { constant }
$$

and the associated kinematic constraint is

$$
\left[\begin{array}{c}
v_{s t} \\
\omega_{s t}^{x} \\
\omega_{s t}^{z}
\end{array}\right]=J_{s t}\left(q_{e}\right) \dot{q}_{e}=0_{5 \times 1},
$$

where $J_{s t}\left(q_{e}\right)$ consists of the first four rows of $J_{s t}$ in (23) and the sixth one.
Since the rotation along the $y$-axis of $R_{s t}$ is free, the contact wrench has five non-zero components as no moment is exerted about the $y$-axis. The wrench can thus be written

$$
F_{s t}=\left(F_{s t}^{f x}, F_{s t}^{f y}, F_{s t}^{f z}, F_{s t}^{m x}, 0, F_{s t}^{m z}\right)^{\prime}
$$

Differentiating (35), and using (19), $\ddot{q}_{e}$ and the nonzero components of $F_{s t}$ can be calculated in terms of $q_{e}, \dot{q}_{e}$ and $u$.

The associated constraints to avoid take-off, slipping, and rotation about the $x$-axis of the foot (i.e., rotation in the frontal plane) are

$$
\begin{aligned}
F_{s t}^{f z} & >0 \\
\left|F_{s t}^{f x}\right| & <\frac{\mu}{\sqrt{2}} F_{s t}^{f z}, \\
\left|F_{s t}^{f y}\right| & <\frac{\mu}{\sqrt{2}} F_{s t}^{f z}, \\
-l_{b} F_{s t}^{f z}<F_{s t}^{m x} & <l_{a} F_{s t}^{f z} .
\end{aligned}
$$

As before, these equations can be grouped as in (29) and a unilateral constraint can be added as in (30), which leads to a domain of admissibility as in (31). In addition, if the state of the robot belongs to the domain of admissibility, then a simplified model can be deduced. From (34), the set of generalized position variables can be taken as $q_{R}=\left(\phi_{s t}^{y}, q^{\prime}\right)^{\prime}$ and the dynamic model can be expressed as ${ }^{11}$

$$
D_{R}(q) \ddot{q}_{R}+C_{R}\left(q_{R}, \dot{q}_{R}\right) \dot{q}_{R}+G_{R}\left(q_{R}\right)=B_{R}(q) u .
$$

The system is always underactuated because no torque is applied about $\phi_{s t}^{y}$.

\subsection{Point-Foot Contact Model}

Consider again the situation in Sect. 3.2 where the foot is flat on the ground and not slipping. If the size of the stance foot is reduced to zero in all dimensions, that is, $L_{a}=L_{b}=l_{a}=l_{b}=h=0$, then the contact with the ground is reduced to a point. This simplifies walking models because there is then only one way for the swing leg to touch the ground, instead of the contact possibly taking place on any edge of the foot.

When realizing a point foot contact as the limiting case of a foot with finite size, two cases can be considered: (a) the (yaw) moment $F_{s t}^{m z}$ goes to zero as well, so the point contact rotates freely about the $z$-axis; or (b), no limit on $F_{s t}^{m z}$ is imposed and there is no rotational slipping of

\footnotetext{
${ }^{11} D_{R}$ is positive definite and the columns of $B_{R}$ remain
} involutive and linearly independent. 
the stance foot $^{12}$ (i.e., no yaw rotation). The latter case is assumed here.

It is now shown that the stance ankle must be passive (i.e., unactuated) when a point foot model is arrived at by letting the size and mass of the stance foot go to zero ${ }^{13}$. To see this, consider the torque balance at the fictitious ankle. For a massless foot, one has

$$
\begin{aligned}
& u_{1}=F_{s t}^{m x}-L F_{s t}^{f z}+h F_{s t}^{f y} \\
& u_{2}=F_{s t}^{m y}+l F_{s t}^{f z}-h F_{s t}^{f x},
\end{aligned}
$$

where $l, L$, and $h$ are the distances along the $x, y$ and $z$ axes between the origin of the reference frame $R_{s t}$ and the ankle. From the ZMP conditions in (28), it follows that both $F_{s t}^{m x}$ and $F_{s t}^{m y}$ must be zero when the size of the supporting foot is zero. From (38), we obtain $u_{1}=$ $u_{2}=0$, and therefore the stance ankle joint must be passive.

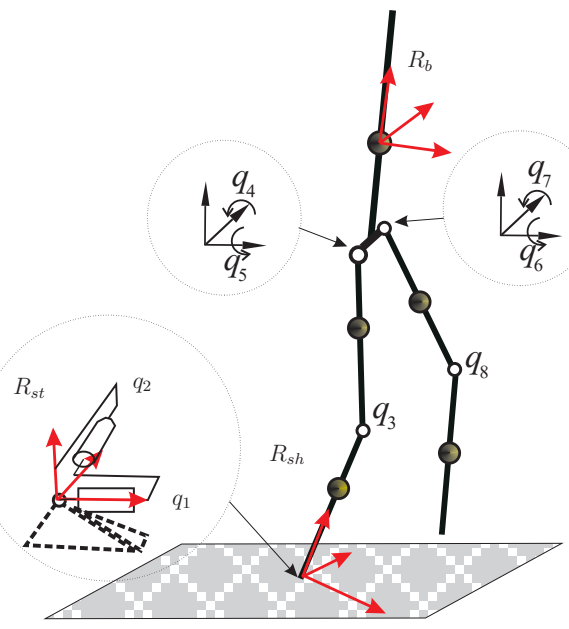

Fig. 4. A reference frame is attached to the shin $R_{s h}$. The motion of the stance shin is limited to the motion produced by a fictitious stance ankle with degrees of freedom $q_{1}=\phi_{s h}^{x}$ and $q_{2}=\phi_{s h}^{y}$.

With the foot reduced to a point, the reference frame used to define the contact constraint is moved to the end of the leg, with its $z$-axis aligned along the shin as depicted in Fig. 4. The reference frame is relabeled as $R_{s h}$; its position and orientation are denoted by $p_{s h}$ and $\phi_{s h}$, respectively. In order to simplify the definition of the holonomic constraint, we choose to define the angles such that the orientation of the frame $R_{s h}$ with respect to the frame $R_{0}$ is ${ }^{0} A_{s h}=\operatorname{Rot}\left(z, \phi_{s h}^{z}\right) \operatorname{Rot}\left(x, \phi_{s h}^{x}\right) \operatorname{Rot}\left(y, \phi_{s h}^{y}\right)$.

\footnotetext{
12 This is analogous to the assumption that the tangential forces lying in a friction cone continue to imply, even for a point contact, no slipping along the plane of the ground contact.

${ }^{13}$ Consequently, models in the literature which treat point feet robots with actuation at the fictitious ankle are assuming a foot of zero mass, but non-zero size.
}

Consequently, the angle $\phi_{s h}^{z}$ defines the orientation of a fictitious foot, and $\phi_{s h}^{x}$ and $\phi_{s h}^{y}$ are the angles of the fictitious ankle. Recalling that we assume no yaw rotation for the stance leg end, the appropriate holonomic constraint is

$$
\eta_{s h}\left(q_{e}\right)=\left[\begin{array}{l}
p_{s h}\left(q_{e}\right) \\
\phi_{s h}^{z}\left(q_{e}\right)
\end{array}\right]=\text { constant. }
$$

It can be shown that the Jacobian $\frac{\partial \eta_{s h}}{\partial q_{e}}$ has full rank.

It is not straightforward to define the contact wrench in the frame $R_{s h}$ because it does not have an axis perpendicular to the ground, making it impossible to express easily the normal component. Thus a frame $R_{s t}$ linked to the fictitious stance foot ${ }^{14}$ is defined. The contact wrench computed at the origin of $R_{s t}$ has four nonzero components, because the only nontrivial moment is about the $z$-axis. It can thus be written as $F_{s t}=$ $\left(F_{s t}^{f x}, F_{s t}^{f y}, F_{s t}^{f z}, 0,0, F_{s t}^{m z}\right)^{\prime}$.

The kinematic constraint corresponding to the holonomic constraint (39) is

$$
\left[\begin{array}{c}
v_{s t} \\
\omega_{s t}^{z}
\end{array}\right]=J_{s t}\left(q_{e}\right) \dot{q}_{e}=0_{4 \times 1}
$$

where $v_{s t}$ and $\omega_{s t}$ are the linear and angular velocity of the shin expressed in the frame $R_{s t}$, and $J_{s t}\left(q_{e}\right)$ consists of the first three rows of $J_{s t}$ in (23) and the sixth one. Differentiating (40), and using (19), $\ddot{q}_{e}$ and the four nonzero components of $F_{s t}$ can be calculated as a function of $q_{e}, \dot{q}_{e}$, and $u$.

The associated constraints to maintain ground contact and to avoid slipping of the foot are

$$
\begin{aligned}
& F_{s t}^{f z}>0, \\
& F_{s t}^{f x}<\frac{\mu}{\sqrt{2}} F_{s t}^{f z}, \\
& F_{s t}^{f y}<\frac{\mu}{\sqrt{2}} F_{s t}^{f z} .
\end{aligned}
$$

There is no longer any constraint of the ZMP type and the fictitious ankle is passive. The equations (41) are grouped as in (29) in order to define the domain of admissibility, as in (31).

When the state of the robot and the control belong to the domain of admissibility, a simplified model is once again deduced. From (39), the set of generalized coordinates

\footnotetext{
${ }^{14}$ This frame is defined with respect to the reference frame $R_{0}$ by the position $p_{s h}$ and the angle $\phi_{s h}^{z}$, thus the use of this frame is coherent with the definition of the holonomic constraint (39).
} 
can be taken as $q_{P}=\left(\phi_{s h}^{x}, \phi_{s h}^{y}, q^{\prime}\right)^{\prime}$ and the dynamic model can be expressed as ${ }^{15}$

$$
D_{P}\left(q_{P}\right) \ddot{q}_{P}+C_{P}\left(q_{P}, \dot{q}_{P}\right) \dot{q}_{P}+G_{P}\left(q_{P}\right)=B_{P}(q) u .
$$

The system is always underactuated because no torque is applied about $\phi_{s h}^{x}$ and $\phi_{s h}^{y}$.

\subsection{Double support phase}

If both legs are in contact with the ground simultaneously, a double support phase occurs. The ground reaction wrench and constraints on it must be considered for each leg, leading to

$D_{e}\left(q_{e}\right) \ddot{q}_{e}+H_{e}\left(q_{e}, \dot{q}_{e}\right)+J_{1}\left(q_{e}\right)^{\prime} F_{1 s t}+J_{2}\left(q_{e}\right)^{\prime} F_{2 s t}=B_{e}(q) u$,

where $J_{i}$ is the Jacobian matrix corresponding to leg $i, i=1,2$. The appropriate holonomic and kinematic constraints associated with how each leg is contacting the ground must be considered, and they can be different for each leg. As in Sect. 3.2-3.4, the type of contact determines the rank of the constraints, which is the same as the number of nonzero components in $F_{1 s t}$ and $F_{2 s t}$, denoted $c_{1}$ and $c_{2}$, respectively. We can group all these constraints and reaction forces into the form (29) and add a unilateral constraint, if present, as in (30). The domain of admissibility is then given as in (31).

Remark 12 If the number of independent velocity constraints associated to the ground contact denoted $c_{12}$ $\left(c_{12} \leq c_{1}+c_{2}\right)$ is greater than 6 , then the robot is over actuated. For a desired motion compatible with the constraints in the robot, an infinite number of control input and reaction wrenches can produce the motion. Control input and reaction wrenches are connected by the dynamic model (43), and control inputs have to be defined such that the reaction wrench belongs to the domain of admissibility. In the specific case of double support with two point-foot contacts, the 6 constraints are not independent, the system is underactuated, and the biped can rotate along the line linking the two points of contact.

\subsection{Impact model}

An impact occurs when a point or collection of points on the robot strikes the ground with a nonzero velocity. Typically, as part of the walking gait design, the contact occurs at either the heel of the swing foot, the sole of the foot, or in the case of a point foot model, the end of the swing leg. The mechanics of contacting bodies is a complicated subject as explained in Kozlov and Treshchev [1992], Brogliato [1999]. Various aspects of it have been addressed for tool use in robotic manipulators;

\footnotetext{
${ }^{15} D_{P}$ is positive definite and the columns of $B_{P}$ remain
} involutive and linearly independent. see Gorinevsky et al. [1997], Siciliano and Villani [1999] and Sciavicco and Siciliano [1996]. Two approximate representations of the contact wrench (forces and torques) have been pursued in the legged robotics literature. One approach assumes the contact is elastic and attempts to model the contact forces due to the deformation of the contacting bodies as in Wei et al. [1993], Plestan et al. [2003]. While elastic models may conceptually capture the actual physical phenomenon, in practice, they tend to introduce a suite of parameters that cannot be readily identified; one is therefore obliged to "guess" reasonable values, introducing uncertainty ${ }^{16}$ and inaccuracy. An alternative approach ${ }^{17}$ assumes the contact is rigid, that is, inelastic.

In a rigid impact, the contact wrench acts over an infinitesimal interval of time and is modeled as a vector of impulses. During the impact, the biped's configuration variables do not change, but the generalized velocities undergo a jump. Most of the rigid impact models used in the locomotion literature are inspired by Hürmüzlü and Marghitu [1994]. The derivation of the model is based on introducing a contact impulse $\delta f_{i m p}$ into the dynamic model (19),

$$
D_{e}\left(q_{e}\right) \ddot{q}_{e}+H_{e}\left(q_{e}, \dot{q}_{e}\right) \dot{q}_{e}=B_{e}(q) u+J\left(q_{e}\right)^{\prime} \delta f_{i m p} .
$$

Assuming that the actuator torques do not contain impulses, (44) is "integrated" over the "duration" of the impact to obtain

$$
D_{e}\left(q_{e}\right)\left(\dot{q}_{e}^{+}-\dot{q}_{e}^{-}\right)=J\left(q_{e}\right)^{\prime} F_{i m p},
$$

where $F_{i m p}:=\int_{t^{-}}^{t^{+}} \delta f_{i m p}(\tau) d \tau$ is the intensity of the impulsive contact wrench over the infinitesimal impact event, $\dot{q}_{e}^{-}$is the generalized velocity just before the impact, and $\dot{q}_{e}^{+}$is the generalized velocity just after the impact. Equation (45) expresses conservation of generalized momentum; see Hürmüzlü and Marghitu [1994]. In the above, it is assumed that the generalized position does not change during the impact, so $q_{e}^{+}=q_{e}^{-}=q_{e}$.

In (45), $\dot{q}_{e}^{-}$is determined as the limit from the left of the state of the robot just before impact, and is thus known. The post-impact velocity $\dot{q}_{e}^{+}$and impact intensity $F_{i m p}$ are unknown. There are thus more unknowns than relations. Completing the model requires that one make $a$ priori assumptions about the nature of the impact. In

\footnotetext{
${ }^{16}$ A compliant ground contact model has been used in Plestan et al. [2003] to check the robustness of a feedback controller computed on the basis of a rigid model. In this case, the uncertainty in the parameters is less important.

17 The user of any contact representation must keep in mind that it is a model of reality and is therefore approximate. The various tradeoffs between ease of use and accuracy must be assessed on a case-by-case basis.
} 
the simplest case ${ }^{18}$, the one analyzed here, the impact model is completed in essentially the same manner that the contact wrench was determined in Sect. 3.2 through Sect. 3.4. Namely, the impact wrench intensity is determined by adding a kinematic constraint on velocity; the form of the constraint is determined by the assumed nature of the impact (flat foot versus heel strike versus point foot, former stance leg releases from the ground versus the robot enters double support, for example); and the validity of the assumed impact must be verified a posteriori by checking that the force and torque components of the resulting impact wrench, and possibly the post-impact velocities, satisfy a set of inequalities.

In order to illustrate the process, assume that the impact of the swing leg with the ground occurs on the sole of the foot (i.e., on a flat foot), the impacting foot neither slips nor rotates, and the former stance leg releases from the ground after the impact. The double support phase is then instantaneous and an impulsive wrench exists on the impacting leg only. The appropriate kinematic constraint is consequently given by (23), computed for the swing leg instead of the stance leg. The kinematic constraint completes the impact model by specifying that

$$
J\left(q_{e}\right) \dot{q}_{e}^{+}=0
$$

In combination with (45), these relations yield the overall impact model (as in equation (27) of Glocker and Pfeiffer [1992]):

$$
\left[\begin{array}{cc}
D_{e}-J^{\prime} \\
J & 0
\end{array}\right]\left[\begin{array}{c}
\dot{q}_{e}^{+} \\
F_{i m p}
\end{array}\right]=\left[\begin{array}{c}
D_{e} \dot{q}_{e}^{-} \\
0
\end{array}\right] .
$$

The matrix on the left-hand side of (47) is square, and it has full rank as long as $J$ has full rank.

Equation (47) shows that the post-impact velocity $\dot{q}_{e}^{+}$ and the impulsive contact wrench $F_{i m p}$ depend linearly on the pre-impact velocity $\dot{q}_{e}^{-}$. Block matrix inversion can be performed using the Schur complement (see Zhang [2005]) to obtain a direct expression for postimpact velocity, namely

$$
\dot{q}_{e}^{+}=\left(I_{(N+6)}-D_{e}^{-1} J^{\prime}\left(J D_{e}^{-1} J^{\prime}\right)^{-1} J\right) \dot{q}_{e}^{-} .
$$

Starting from a reduced model of the robot before impact, for example, $q^{-}, \dot{q}^{-}$determined from (32) or (42), the corresponding holonomic constraint yields the complete state of the robot $q_{e}^{-}, \dot{q}_{e}^{-}$just before impact. Solving for the post-impact velocity (48) and projecting it

\footnotetext{
${ }^{18}$ A contact event does not necessarily correspond to a holonomic constraint. The foot could slide after impact, for example. Such cases are more difficult to model.
}

down to the reduced model of the ensuing phase gives a reduced impact model written in the form

$$
\dot{q}^{+}=\Delta(q) \dot{q}^{-}
$$

Almost every paper on legged locomotion contains a version of this equation.

It should be noted that the expression (49), which suppresses the use of the complete state of the robot, hides the fact that the holonomic constraints before and after impact are not identical (at the very least, the former swing leg is now in contact with the ground). It is a crucial step, which is omitted in many papers, to verify that the post-impact velocity and the impulsive contact wrench are compatible with the holonomic constraint assumed after impact. In particular, since no constraint was imposed on the former stance leg, it must be the case that the vertical component of the post-impact foot velocity is positive (i.e., the foot is lifting from the ground after the impact). Next, the friction pyramid should be verified with $F_{i m p}$ replacing $F_{s t}$ (i.e., the foot is not slipping post-impact), and finally, the ZMP conditions need to be checked for $F_{i m p}$ (i.e., the foot is rotating appropriately or not about one of its edges, post impact). If any of these conditions are violated, then the assumed impact model was invalid, another set of constraints must be posed, and the entire process repeated; see Hürmüzlü and Marghitu [1994].

The models for an impact occurring on the heel or for the impact of a point foot, while assuming an instantaneous double support phase, are developed in the same manner, using velocity constraints on the swing leg that are analogous to (35) and (40) and the appropriate nonzero components of $F_{i m p}$. A detailed derivation for a planar point foot contact can be found in Westervelt et al. [2007a]; the 3D case is very similar as shown in Chevallereau et al. [2009b].

Remark 13 If the former stance leg remains on the ground after the impact, reaction forces and velocity constraints must be applied to both legs, similar to the double support model in Sect. 3.5. It has been observed that it may be difficult to satisfy the constraints (foot remains on the ground, no slipping, ZMP condition) associated to each impulsive contact wrench. In particular, Miossec and Aoustin [2006] have shown for the planar robot Rabbit that the former stance leg remains on the ground only in the case of an impactless ${ }^{19}$ contact.

Remark 14 In general, in point feet models, the impacting leg is assumed to stay on the ground, although

\footnotetext{
${ }^{19}$ If the pre-impact velocity satisfies the assumed postimpact condition of contact, i.e $J \dot{q}_{e}^{-}=0$, the velocity does not change at impact, that is, $\dot{q}_{e}^{+}=\dot{q}_{e}^{-}$, and $F_{i m p}=0$. The motion is called impactless.
} 
it can slide in the presence of insufficient friction, as in Rodriguez and Bowling [2013]. A point foot that was in rigid contact before impact can either lift-off, stay put, or slide. Thus, for the impact of a walking biped with point feet, six impact cases have to be considered. In the case of a robot with finite feet, many more cases have to be considered since the foot can be flat on the ground or can be rotating about one of its edges, or even one of its corners. Multi-point models of impact are studied in Rodriguez and Bowling [2012]. Additional complexity could come in the form of Zeno solutions, where the foot rebounds an infinite number of times over a finite interval; see Or and Ames [2008, 2009], Lamperski and Ames [2008, 2013], Goebel et al. [2009]. Typically, control solutions are sought which avoid such behavior.

\subsection{Hybrid Models}

An overall hybrid model of a walking gait is constructed by first enumerating a list of dynamic models, $1 \leq \mathrm{i} \leq N_{p}$, corresponding to allowed phases in the gait. In general, a directed graph of possible transitions among phases must be constructed. When studying periodic gaits, it is much simpler and more common to specify a cyclic graph, that is, a temporal order of the form $1 \rightarrow 2 \rightarrow \cdots \rightarrow N_{p} \rightarrow 1$. By imposing a temporal ordering, we are assuming a priori that the flow intersects a specific subset of the domain's boundary; this must be taken into account when a control law is designed and then verified when the model is analyzed for stable orbits. Specific examples of cyclic graphs are worked out in Sects. 5 and 7.

In the following, we suppose that phase i corresponds to one of the single support models (32), (37), or (42), or a double support model as discussed in Sect. 3.5. Let the configuration space be $Q_{\mathrm{i}}$ and the state space be $\mathcal{X}_{\mathrm{i}}=T Q_{\mathrm{i}}$. The state variable control model is then

$$
\begin{aligned}
\frac{d}{d t}\left[\begin{array}{c}
q_{\mathrm{i}} \\
\dot{q}_{\mathrm{i}}
\end{array}\right]= & {\left[\begin{array}{c}
\dot{q}_{\mathrm{i}} \\
-D_{\mathrm{i}}^{-1}\left(q_{\mathrm{i}}\right)\left[C_{\mathrm{i}}\left(q_{\mathrm{i}}, \dot{q}_{\mathrm{i}}\right) \dot{q}_{\mathrm{i}}+G_{\mathrm{i}}\left(q_{\mathrm{i}}\right)\right]
\end{array}\right]+} \\
& {\left[\begin{array}{c}
0 \\
D_{\mathrm{i}}^{-1}\left(q_{\mathrm{i}}\right) B_{\mathrm{i}}\left(q_{\mathrm{i}}\right)
\end{array}\right] u_{\mathrm{i}} }
\end{aligned}
$$

where $u_{\mathrm{i}} \in \mathcal{U}_{\mathrm{i}} \subset \mathbb{R}^{m_{\mathrm{i}}}$ is the vector of actuator torques. Defining $x_{\mathrm{i}}=\left(q_{\mathrm{i}}^{\prime}, \dot{q}_{\mathrm{i}}^{\prime}\right)^{\prime}$ yields

$$
\dot{x}_{\mathrm{i}}=f_{\mathrm{i}}\left(x_{\mathrm{i}}\right)+g_{\mathrm{i}}\left(x_{\mathrm{i}}\right) u_{\mathrm{i}}
$$

Recall that the various Lagrangian models come with a domain of admissibility, such as (31), arising from the ground contact conditions. Let the element from the constraint vector (30) corresponding to the appropriate edge for transition into phase $\mathrm{i}+1$ be $H_{\mathrm{i}}^{\mathrm{i}+1}\left(x_{\mathrm{i}}, u\right)$. In general, the transition condition depends on the actuator torques as well as the system's state. For simplicity, it is often supposed that $H_{\mathrm{i}}^{\mathrm{i}+1}$ and $\dot{H}_{\mathrm{i}}^{\mathrm{i}+1}$ do not depend on $u$ so that a switching surface is given by

$$
\mathcal{S}_{\mathrm{i}}^{\mathrm{i}+1}=\left\{x_{\mathrm{i}} \in \mathcal{X}_{\mathrm{i}} \mid H_{\mathrm{i}}^{\mathrm{i}+1}\left(x_{\mathrm{i}}\right)=0, \dot{H}_{\mathrm{i}}^{\mathrm{i}+1}\left(x_{\mathrm{i}}\right)<0\right\}
$$

Note that as in Sect. 2.3, addition modulo the number of phases $N_{p}$ is used, so that $N_{p}+1=1$.

To complete the specification of a hybrid model, the reset map $\Delta_{i}^{\mathrm{i}+1}: \mathcal{S}_{\mathrm{i}}^{\mathrm{i}+1} \rightarrow \mathcal{X}_{\mathrm{i}+1}$ must be defined. If the transition condition in (52) corresponds to an impact, such as the swing leg height above the ground going to zero, then the reset map is computed as in (49). In other cases, when impacts are not involved, the transition map is typically determined by inserting the state of the robot into the state space of the full model (13), and then projecting down to the state space of the reduced model of the next phase.

Putting all of this together results in a hybrid control system of the form

$$
\Sigma:\left\{\begin{array}{c}
\mathcal{X}=\left\{\mathcal{X}_{\mathrm{i}}\right\}_{\mathrm{i}=1}^{N_{p}}: \mathcal{X}_{\mathrm{i}} \subset \mathbb{R}^{n_{\mathrm{i}}} \\
\mathcal{U}=\left\{\mathcal{U}_{\mathrm{i}}\right\}_{\mathrm{i}=1}^{N_{p}}: \mathcal{U}_{\mathrm{i}} \subset \mathbb{R}^{m_{\mathrm{i}}} \\
\mathcal{F} \mathcal{G}=\left\{\left(f_{\mathrm{i}}, g_{\mathrm{i}}\right)\right\}_{\mathrm{i}=1}^{N_{p}}: \dot{x}_{\mathrm{i}}=f_{\mathrm{i}}\left(x_{\mathrm{i}}\right)+g_{\mathrm{i}}\left(x_{\mathrm{i}}\right) u_{\mathrm{i}} \\
\mathcal{S}=\left\{\mathcal{S}_{\mathrm{i}}^{\mathrm{i}+1}\right\}_{\mathrm{i}=1}^{N_{p}}: \mathcal{S}_{\mathrm{i}}^{\mathrm{i}+1}=\left\{x_{\mathrm{i}} \in \mathcal{X}_{\mathrm{i}} \mid H_{\mathrm{i}}^{\mathrm{i}+1}\left(x_{\mathrm{i}}\right)=0,\right. \\
\left.\dot{H}_{\mathrm{i}}^{\mathrm{i}+1}\left(x_{\mathrm{i}}\right)<0\right\} \\
\Delta=\left\{\Delta_{\mathrm{i}}^{\mathrm{i}+1}\right\}_{\mathrm{i}=1}^{N_{p}}: x_{\mathrm{i}+1}^{+}=\Delta_{\mathrm{i}}^{\mathrm{i}+1}\left(x_{\mathrm{i}}^{-}\right) .
\end{array}\right.
$$

As with uncontrolled hybrid models, the hybrid control model can be written in the form of a tuple that is more consistent with the literature on hybrid systems, namely,

$$
\mathscr{H} \mathscr{C}=\Sigma=(\Gamma, \mathcal{X}, \mathcal{U}, \mathcal{S}, \Delta, \mathcal{F} \mathcal{G})
$$

For the formal definition of hybrid systems stated in this form, and defined on more general graphs, see Sinnet and Ames [2009a] and Ames et al. [2011].

Remark 15 A typical hybrid model would include phases for support on both the left and right legs. When studying walking gaits with left-right symmetry, a common "trick" in the field is to develop a model of the robot for one of the legs in contact with the ground, say the left one, and then to "swap" or relabel angles after swing leg impact in order to propagate the dynamics for the ensuing step. The interest of doing this is that it reduces the number of phases by half. This "trick" is particularly common and straightforward for planar models. For 3D models, it is a little more involved as one must "flip" the sign on the hip width and "flip" the sign convention for all angles that are not in the sagittal plane. When using 
this more economical model of a biped, a state relabeling procedure is included as part of the reset map at leg impact.

\section{Approaches to Feedback Control of Locomo- tion}

Over the past fifty years, research into bipedal robots has come from a variety of perspectives: from passive walkers with simple mechanical designs to advanced multifunctional humanoids. Myriad control designs have been introduced using models which vary from the simplified representations shown in Fig. 5 to full-dimensional dynamic models as developed in Sect. 3. Proponents of simplified models point to the benefits of more tractable analysis, enhanced insight, and faster control law computation, whereas proponents of more complex models point to enhanced predictive ability and formal guarantees on stability. In this section, some of the more common approaches to bipedal gait generation are examined with a particular emphasis on how the underlying modeling paradigms affect control design. The book Westervelt et al. [2007a] and the review paper Hürmüzlü et al. [2004] provide an extensive overview of the state of the art up to early 2006; further information is available in Full and Koditschek [1999], Holmes et al. [2006], Wisse and van der Linde [2007], Kuo [2007], Siciliano and Khatib [2008], Chevallereau et al. [2009a], Sadati et al. [2012] and the references therein.

\subsection{Zero Moment Point and Linear Inverted Pendulum Models}

One of the most common control methods for bipedal locomotion is the ZMP control strategy. Recall from Sect. 3.2 that the ZMP is the point on the ground at which the reaction forces acting between the ground and the foot produce no horizontal moment. Traditionally, ZMP control strategies achieve walking by planning the motion of a robot's CoM such that the ZMP remains strictly within the convex hull of the stance foot in the case of single support (or convex hull of the stance feet, in the case of double support). Under this condition on the $\mathrm{ZMP}$, the stance foot remains flat on the ground and immobile (not rotating) - much like the base of a traditional manipulator robot - and hence the robot will not topple; see, e.g., Yamaguchi et al. [1999].

In the special case of the Linear Inverted Pendulum Model (LIPM), the ZMP can be expressed explicitly in terms of the dynamics of the CoM via a linear ordinary differential equation (ODE). There are key assumptions permitting this simplification, including representing a robot as a point mass with massless telescoping legs. Additionally, the height of the CoM is assumed constant throughout a step. Under these conditions, Kajita and Tani [1991] showed that the robot model reduces to the
LIPM. Because of their simplicity when combined, the ZMP control method and the LIPM have historically been tightly connected. While the model found its origins in the study of human posture and balance (e.g., Geursen et al. [1976], Winter [1995], Patton et al. [1999]), it has also been the subject of much attention in bipedal walking; see, for example, Miura and Shimoyama [1984], Kajita et al. [2001, 2010].

Many of the early experimental results of bipedal robotic walking came from Japan, where Kato began working on the WABOT series of humanoid robots around 1970. A full-scale anthropomorphic robot, WABOT-1, was reported in Kato et al. [1974] and it demonstrated primitive, statically stable walking while transporting objects with its hands. Over a decade later, the ZMP technique was first demonstrated in practice on the WL10RD biped in Takanishi et al. [1985]. Interest in walking humanoid robots has continued to grow with researchers from around the world frequently developing newer generations like WABIAN-2, ASIMO, HRP-4, KHR-3, and Johnnie (Ogura et al. [2006], Sakagami et al. [2002], Kaneko et al. [2011], Park et al. [2005], Pfeiffer et al. [2002]).

In spite of the widespread success of ZMP methods, there are recognized limitations. Gaits designed using this method generally do not take impacts into account, and thus the swing foot trajectory must be designed so that it will impact the ground with minimal velocity, which can be hard to achieve. In addition, it is known that meeting the ZMP condition is not sufficient for asymptotic stability of a periodic walking motion (see Choi and Grizzle [2005]). Gait generation using ZMP has taken many forms: Kurazume et al. [2003] used analytical solutions to the ZMP dynamics; Nagasaka et al. [1999] used the optimal gradient method; Kajita et al. [1992] examined potential energy conserving orbits; Lim et al. [2002] computed ZMP-consistent trajectories offline and stabilized them using trunk motion; and Nishiwaki et al. [2002] generated ZMP-consistent trajectories in real-time while walking. Additional information on ZMP-based methods and related ground reference points is given in Goswami [1999], Vukobratović and Borovac [2004], Vukobratović et al. [2006], Popovic et al. [2005].

\subsection{Nonlinear Inverted Pendulum Models}

In order to overcome limitations resulting from the simplicity of the LIPM model, researchers have considered more complex models. Park and Kim [1998] explored the Gravity Compensated LIPM which adds an additional point mass at the location of the swing foot to achieve higher modeling accuracy. In Pratt and Drakunov [2007], the requirement of constant CoM height on the LIPM was relaxed leading to a nonlinear inverted pendulum model. In another common model, a flywheel is added to the inverted pendulum; examples can be found throughout the literature: Stephens [2011] used it for posture 


\section{Increasing Model Complexity}

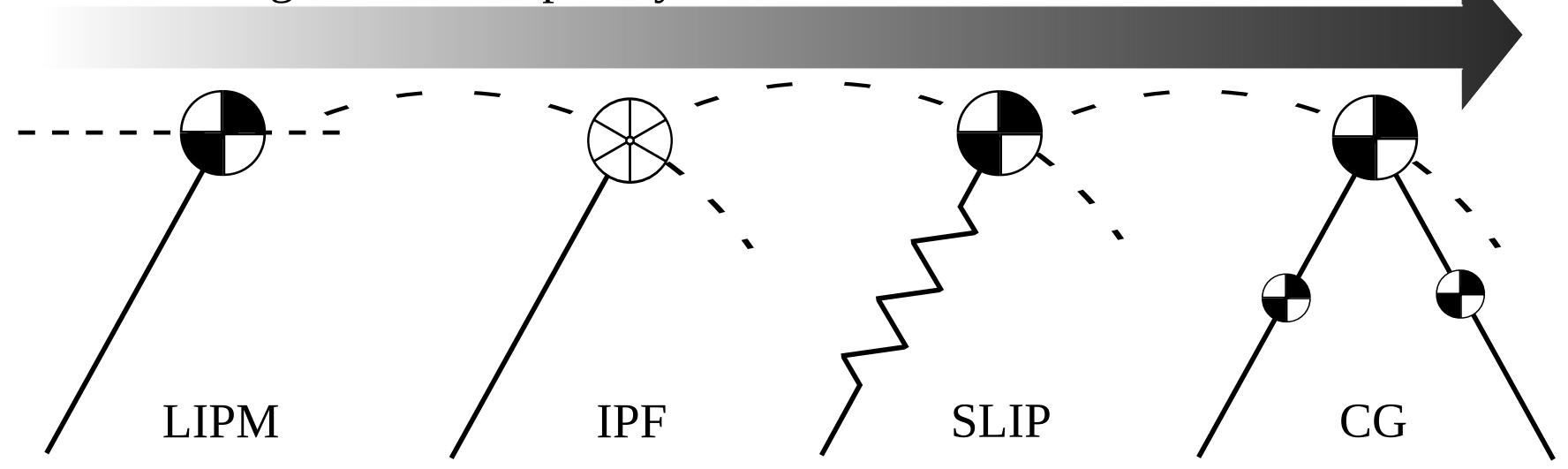

Fig. 5. Four low-dimensional models that are frequently used as approximate representations of walking robots. From left to right: the Linear Inverted Pendulum Model (LIPM) lumps the mass of the robot at a point moving at a constant height and assumes massless legs; the Inverted Pendulum with Flywheel (IPF) relaxes the assumption on constant height and adds a flywheel to account for internal angular momentum; the Spring-Loaded Inverted Pendulum (SLIP) adds a spring to model a robot's legs as a massless pogo stick; and the Compass-Gait Biped (CG) treats a robot as a double pendulum with lumped masses on the stance and swing legs.

control, Takenaka et al. [2009] used it to with on-line error compensation to mitigate the effect of modeling errors on gait generation, and Komura et al. [2005] used it to simulate pathological gaits. The various pendulum models have been widely used in analysis of push recovery and balance (Takanishi et al. [1990], Hof et al. [2005], Hyon et al. [2007], Stephens [2007]).

Pratt et al. [2006] considered a flywheel model in order to present the idea of the capture point - a point on the ground on which a biped can step and come to a complete (upright) stop without falling over; additional information on capture points can be found in Koolen et al. [2012], Pratt et al. [2012]. The capture point (Pratt and Tedrake [2006]) has gained recognition as a convenient method for stabilizing a biped. This method, which considers a robot as an inverted pendulum with a flywheel, has been used not only for standing but for robust walking as well. Because the model makes many simplifying assumptions, the capture regions - the set of all capture points - can have a large error and this has motivated the combining of capture point with learning in, e.g., Rebula et al. [2007].

\subsection{Raibert Hoppers and SLIP Models}

Raibert observed that hopping and running can be represented by a low-dimensional model with springs and built a pneumatically actuated planar monopod with a pogo-stick (springy) leg that was able to run ${ }^{20}$ at a speed of $1 \mathrm{~m} / \mathrm{s}$ (Raibert [1984], Raibert and H. Brown,

\footnotetext{
${ }^{20}$ Roughly speaking, running consists of a stance phase with one foot on the ground followed by a flight phase with no feet on the ground; hence hopping on one leg and running on two legs are closely related.
}

Jr. [1984], Raibert [1986]). This was followed by a 3D hopper that was able to run without being constrained to the sagittal plane ([Raibert, 1986, Chap. 3], Raibert et al. [1984]) as well as multi-legged robots (Raibert et al. [1986], Raibert [1990], Hodgins and Raibert [1991]).

This body of work gave rise to the Spring-Loaded Inverted Pendulum (SLIP) model, which has been shown to approximate the body center-of-mass (CoM) motion during steady-state running gaits of a wide diversity of terrestrial animals (Blickhan [1989], McMahon and Cheng [1990], Farley et al. [1993], Full and Farley [2000], Dickinson et al. [2000], Seyfarth et al. [2001]). Successful running robots, such as the Planar Hopper, ARL Mono pod II and CMU Bowleg Hopper, also exhibit SLIP model behavior (Raibert [1986], Zeglin and Brown [1998], Ahmadi and Buehler [2006]).

The control of these robots has been based on Raibert's original control ideas, which can be decomposed into three subtasks dedicated to (a) forward propulsion of the robot at the desired speed, (b) regulation of the vertical hopping height of the body, and (c) keeping the body at a desired posture (Raibert [1984], [Raibert, 1986, Chapter 2]). To control the forward speed, the controller places the toe at a desired position with respect to the center of mass during flight. To regulate the hopping height, the length of the leg at the bottom of the stance phase is adjusted by giving a fixed amount of thrust. Finally, to control the pitch attitude of the body, the controller employs hip torque during stance. The inclusion of springs into legged robots with revolute knees seems to have started with spring flamingo and spring turkey as described in Hollerbach et al. [1992], Pratt and Pratt [1999], Pratt [2000], Pratt et al. [2001]. These robots used a type of series elastic actuator (SEA) designed for 
force control as opposed to energy storage. The recent COMAN robot discussed in Kormushev et al. [2011] includes passive compliance to reduce energy consumption during walking.

\subsection{Passive Walkers and Compass-Gait Biped}

At the other end of the spectrum, instead approximating complex bipeds with simplified models, some researchers have opted to design robots with dynamics that approximately realize a simplified model. For these bipeds, dynamic stability is achieved as much as possible through mechanical design instead of feedback control. Though comparatively less complex in terms of the models studied, many of the formal methods developed on simple bipeds still enjoy use in complex systems. However, the simpler design facilitates more complete modeling and, indeed, control researchers who follow this path often consider impact dynamics, thereby taking into account the full hybrid model which is generally omitted from analysis of controllers designed using the LIPM.

Mochon and McMahon [1980] concluded that the swing phase of human walking is similar to a double pendulum, pointing to the passive nature of human walking and the importance of mechanism design. In the late 80's, McGeer analyzed and built planar, passive bipedal walkers, i.e., no actuation, which could walk stably down shallow slopes, starting with the compass gait walker (which is simply an inverted pendulum) in McGeer [1990a] and later adding knees in McGeer [1990b]. This gave rise to the terminology passive dynamic walking. Subsequently, robots with this general principle at their core have been constructed, as described in Collins et al. [2005], based on injecting small amounts of energy into passive-type bipeds. The result is very "human-looking" walking, but the remarkable elegance and economy of these walkers comes at the cost of poor ability in achieving tasks other than walking at a fixed speed; they cannot climb stairs, pause, turn or run.

Additional work was later done to analyze the properties of passive dynamics walkers, for instance, in Espiau and Goswami [1994], Garcia et al. [1998], Borzova and Hurmuzlu [2004]. In terms of control, Spong [1999] looked at passive dynamic walking with energy-based methods to design passivity-based control strategies such as controlled symmetries, introduced in Spong and Bullo [2005]. Other important contributions to passive dynamic walking are given in Kuo [1999, 2002], Anderson et al. [2005], Wisse and van der Linde [2007].

\subsection{Quadratic Programs and Lyapunov Funnels}

Early implementations of ZMP-based controllers used offline trajectory optimization to generate center of mass trajectories on the basis of the LIPM and generally ignored impact dynamics in the control design. Modern methods have achieved improved control by formulating the control problem as a quadratic program (QP), allowing gait replanning on the fly and improving stability properties (Kudoh et al. [2002], Stephens and Atkeson [2010], Herdt et al. [2010]).

Similarly, sums of squares methods, also formulate trajectory generation as a convex optimization (Tedrake et al. [2010]). One elegant method which provides formal guarantees on stability is outlined in Majumdar et al. [2013]. The procedure investigates the notion of controllability, composing sequential funnels (verified with sum of squares Lyapunov inequalities), which each lead to a predefined goal set. This allows one to create a trajectory with guaranteed stability properties: at any given point in time, the system is within one of the known funnels (regions of attraction). By using the sum of squares formulation, the trajectory optimization becomes more tractable, making verification of stability feasible for low-dimensional models.

\section{Controlling Underactuated Bipedal Locomo- tion via Virtual Constraints and Hybrid Zero Dynamics}

This section overviews an approach to achieving asymptotically stable bipedal locomotion in the presence of underactuation. As discussed in Sect. 3, the contact conditions between the robot and the ground are extremely important for the control of a biped. Moreover, the most difficult conditions to satisfy are the ZMP conditions, as illustrated in (28) and (36), for example. With this as motivation, the work in this section is tailored to feedback control of a biped with the point-foot contact model described in Sect. 3.4, corresponding to the limiting case of a robot with feet, as the size of the feet decreases to zero. If this robot can be controlled with no actuation at the point of contact, then flat-footed walking with actuated feet (of any size) can be accomplished with (arbitrarily small) torques that will respect the constraint of no rotation about an edge of the stance foot, thereby removing an important obstacle to previous studies of walking. Once a gait consisting only of point feet walking of a 3D robot can be controlled, then, based on previous work in planar robots in Chevallereau et al. [2008] and Choi and Grizzle [2005], there is good reason to believe that a gait consisting of a more complete walking cycle, heel strike, flat foot, toe roll, can be realized in a stable manner. Indeed, a freely rotating point of contact is potentially more difficult to control than the heel-strike or toe-roll phases of a human walking gait, which correspond to rotation about a single axis instead of two axes. Finally, work in Chevallereau et al. [2008] shows that even in the case of flat-footed walking with an actuated ankle, if the center of pressure of the ground reaction forces on the stance foot is actively controlled in order to avoid foot rotation, the corresponding control problem is underactuated. For these reasons, walking with unactuated point 
feet presents an interesting test case for any control design methodology.

In the following, a constraint is said to be virtual if it is achieved through feedback control instead of through physical connections, such as gears or contact conditions with the environment. Virtual constraints can be used to synchronize the evolution of a robot's links throughout a gait. A connection with the now-classical notion of zero dynamics will become clear during the presentation, with one novelty being the extension of the notion of zero dynamics to a class of hybrid models that occur in bipedal locomotion. Virtual constraints and hybrid zero dynamics originated in the study of underactuated, planar bipedal locomotion in Grizzle et al. [2001], Westervelt et al. [2003]; a synthesis of these methods can be found in Westervelt et al. [2007a]. The methods are currently being extended to underactuated 3D robots; see Chevallereau et al. [2009b]. The utility of virtual constraints and hybrid zero dynamics has been experimentally verified for planar bipedal robots Chevallereau et al. [2003], Westervelt et al. [2004], Sreenath et al. [2011b]. The 3D results are still in their infancy and much work remains to be done.

\subsection{Virtual Constraints}

Any attempt to describe a walking gait, even something as simple as the difference between human-like walking (knees bent forward) and bird-like walking (knees bent backward), or the torso being upright versus leaning forward, leads to a description of the posture or shape of the robot throughout a step. In other words, a description of walking involves at least a partial specification of the path traced out in the configuration space of the robot. Fig. 6 illustrates this idea for the simple case of a planar biped evolving in the sagittal plane. The same idea applies to a $3 \mathrm{D}$ robot, with the addition of coordinates parameterizing the robot's evolution in the frontal plane and its yaw rotation.

To formalize this approach to locomotion control, suppose that $\mathcal{O}$ is a periodic orbit corresponding to a walking gait with $N_{p} \geq 1$ continuous phases ${ }^{21}$ (or domains). Let $q=\left(q_{1}, \cdots, q_{N}\right)$ be a set of generalized coordinates for a particular phase $\mathcal{X}_{\mathrm{i}}$ of the gait, and let $h_{0}(q)$ be functions of the generalized coordinates that are to be controlled, such as the knee and hip angles illustrated in Fig. 6. Let $\theta(q)$ be a function of the generalized coordinates that is strictly monotonic ${ }^{22}$ on $\mathcal{O}_{\mathrm{i}}:=\mathcal{O} \cap \mathcal{X}_{\mathrm{i}}$, and

\footnotetext{
${ }^{21}$ The development here focuses on control with full-state feedback within the continuous phases. Control at the discrete transitions is addressed in Westervelt et al. [2007a]. Observer design is discussed in Grizzle et al. [2007].

${ }^{22} \dot{\theta}$ strictly positive or negative on the orbit. Functions which commonly satisfy this include the horizontal position of the robot's hips with respect to an inertial frame, or the absolute angle of the line connecting the stance leg end to the hip.
}

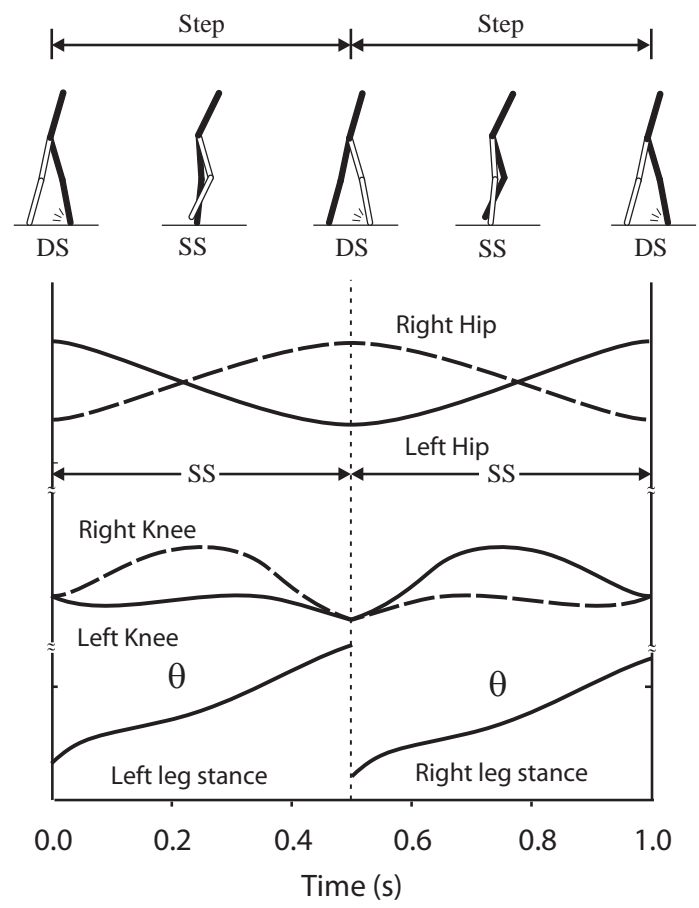

Fig. 6. Illustration of the concept of virtual constraints. Depicted are the relative knee and hip angles versus time for a planar, point-foot walker over a symmetric periodic gait. The gait is comprised of alternating phases of single support (SS), described by a Lagrangian model, and double support (DS), assumed to be instantaneous. The variable $\theta$ (taken here as the angle with respect to the ground of the line connecting the stance leg end to the hip) is strictly monotonically increasing in each continuous phase of the gait. Plotting the joint angles versus $\theta$ provides a graph of the virtual constraints (54) for the relative relative knee and hip angles. The same idea is applicable to $3 \mathrm{D}$ walkers.

express the controlled variables as a function ${ }^{23} h_{d}(\theta)$ so that, on the periodic orbit,

$$
\left.\left(h_{0}(q)-h_{d}(\theta)\right)\right|_{\mathcal{O}_{\mathrm{i}}} \equiv 0 .
$$

Off the orbit,

$$
y=h(q):=h_{0}(q)-h_{d}(\theta)
$$

is nonzero, and a natural objective is therefore to design a feedback controller that drives $h(q)$ asymptotically to zero. The function $h(q)$, or more correctly, the relation $h(q)=h_{0}(q)-h_{d}(\theta)=0$, is called a virtual constraint. The number of constraints will be assumed equal to the number of inputs in all that follows.

Two questions naturally arise:

An analysis question: For a given periodic orbit $\mathcal{O}$ and selection of virtual constraints (in general, different

\footnotetext{
${ }^{23}$ Always possible when $\theta$ is strictly monotonic.
} 
for each domain), when will driving $y$ in (55) asymptotically to zero render the orbit stable (resp., asymptotically stable, or exponentially stable)?

A synthesis question: how to design virtual constraints, and feedback controllers that asymptotically impose them, which together yield an asymptotically stable periodic orbit meeting physically motivated requirements such as: energy efficiency; the robot walks at a desired speed; and the reaction forces at the leg end respect required unilateral constraints?

Addressing the first question leads to the notion of the hybrid zero dynamics, which will be outlined in Sects. 5.2 and 5.3. A finite parametrization of possible paths $h_{d}(\theta, \alpha)$ via Bézier polynomials and parameter optimization have been employed to address the second question; see Sect. 5.4. An illustration is given in Sect. 5.5 .

\subsection{Zero Dynamics}

We focus first on the dynamics within a given continuous phase $\mathcal{X}_{\mathrm{i}}$ of a gait model. Let once again $q=$ $\left(q_{1}, \cdots, q_{N}\right)$ be a set of generalized coordinates and suppose the model is given by

$$
D(q) \ddot{q}+C(q, \dot{q}) \dot{q}+G(q)=B u,
$$

where $D(q)$ is the inertia matrix, $C(q, \dot{q})$ contains Coriolis and centrifugal terms, $G(q)$ is the gravity vector, and $B$ is an $N \times N_{a}$ constant matrix with rank $N_{a}<N$. Letting $x=(q, \dot{q})$, and defining $f$ and $g$ in the standard manner, the mechanical model is expressed in state variable form as

$$
\dot{x}=f(x)+g(x) u .
$$

It is noted in passing that the distribution generated by the columns of $g$ is automatically involutive because the matrix $B$ in (56) is constant.

Let the output be defined as in (55) and assume it has vector relative degree ${ }^{24}$ two. According to Isidori [1995], the zero dynamics manifold is then

$$
\mathcal{Z}_{\mathrm{i}}:=\left\{x \in \mathcal{X}_{\mathrm{i}} \mid h(x)=0, L_{f} h(x)=0\right\}
$$

A feedback control law rendering $\mathcal{Z}_{\mathrm{i}}$ forward invariant

\footnotetext{
${ }^{24}$ The assumption of vector relative degree two is for convenience and works well in applications. A uniform vector relative degree of $k$ is treated in Morris and Grizzle [2009]; a case with a non-uniform vector relative degree is treated in Poulakakis and Grizzle [2009]. Even the assumption of a vector relative degree could be relaxed to dynamic inputoutput linearizability, for example, but we have had no need to do this in the applications studied to date.
}

and attractive is

$$
u=u^{*}(x)-\left[L_{g} L_{f} h(x)\right]^{-1}\left(\frac{1}{\epsilon^{2}} K_{p} h(x)+\frac{1}{\epsilon} K_{d} L_{f} h(x)\right)
$$

with

$$
u^{*}(x)=-\left[L_{g} L_{f} h(x)\right]^{-1} L_{f}^{2} h(x),
$$

where $K_{p}>0, K_{d}>0$ and $\epsilon>0$. In applications, $\epsilon>0$ is used to adjust the rate of convergence ${ }^{25}$ to the zero dynamics manifold.

The feedback $u^{*}$ renders $\mathcal{Z}_{\mathrm{i}}$ invariant under the closedloop vector field $f+g u^{*}$ defined on the continuous phase $\mathcal{X}_{\mathrm{i}}$. The zero dynamics vector field is the restriction

$$
f_{\text {zero }}:=f+\left.g u^{*}\right|_{\mathcal{Z}_{\mathrm{i}}}
$$

The phase- $\mathcal{X}_{\mathrm{i}}$ zero dynamics is then

$$
\dot{z}=f_{\text {zero }}(z)
$$

for $z \in \mathcal{Z}_{\mathrm{i}}$

Because $\mathcal{Z}_{\mathrm{i}}$ has been designed without consideration of the reset maps in the hybrid model, there is no reason for it to be impact invariant, and hence hybrid invariant. The next section discusses a means developed in Morris and Grizzle [2009] for achieving hybrid invariance without imposing stringent conditions on the feedback designs in neighboring domains or conditions on the reset maps. It is based on realizing the virtual constraints $h$ in (55) as one member of a parameterized family of virtual constraints. The parameters in the constraints are updated upon transition into domain $\mathcal{X}_{\mathrm{i}}$ so as to achieve invariance, while preserving the original orbit used in the design of $h$.

\subsection{Hybrid Invariance}

We continue to focus on a domain $\mathcal{X}_{\mathrm{i}}$ where a feedback law has been constructed as in Sect. 5.2. We suppose for the moment that a feedback law has been defined in each of the other domains; this may be achieved by following the virtual constraints and zero dynamics approach of the previous section, or by any other approach as long as (i) $\mathcal{O}$ is a periodic orbit of the model and (ii) the closedloop model is smooth enough for the maps defined below to be $C^{1}$ in a neighborhood of the periodic orbit $\mathcal{O}$. These

\footnotetext{
${ }^{25}$ In many concrete examples, the reset map tends to amplify the distance from a periodic orbit and thus the continuous phase dynamics must be sufficiently contractive in order to achieve orbital stability. The parameter $\epsilon$ is used for this purpose. The assumptions on the feedback parameters guarantee that $\lambda^{2}+\frac{1}{\epsilon} K_{d} \lambda+\frac{1}{\epsilon^{2}} K_{p}$ is Hurwitz.
} 
assumptions allow a system with impulse effects to be associated with domain $\mathcal{X}_{\mathrm{i}}$ by defining

$$
\Sigma:\left\{\begin{aligned}
\dot{x} & =f(x)+g(x) u & x^{-} & \notin \mathcal{S} \\
x^{+} & =\Delta\left(x^{-}\right) & x^{-} & \in \mathcal{S}
\end{aligned}\right.
$$

where $x \in \mathcal{X}_{\mathrm{i}}, \mathcal{S}=\mathcal{S}_{\mathrm{i}}^{\mathrm{i}+1}$, and $\Delta=\Delta_{\mathrm{i}-1}^{\mathrm{i}} \circ P_{\mathrm{i}-1} \circ \cdots \circ$ $P_{1} \circ P_{N_{p}} \circ \cdots P_{\mathrm{i}+1}$, where $P_{\mathrm{i}}$ are the generalized Poincaré maps defined in (6).

The manifold $\mathcal{Z}_{\mathrm{i}}$ is forward invariant under $f_{\text {zero }}=f+$ $g u^{*}$ and $\mathcal{O}$ is a solution of the zero dynamics. However, $\mathcal{Z}_{\mathrm{i}}$ is not necessarily invariant under the transition map $\Delta$, that is, the condition $\Delta\left(\mathcal{Z}_{\mathrm{i}} \cap \mathcal{S}\right) \subset \mathcal{Z}_{\mathrm{i}}$ does not hold in general.

Reference Morris and Grizzle [2009] provides a constructive procedure for determining an open neighborhood $\mathcal{B}$ of the origin in $\mathbb{R}^{2 N_{a}}$, a smooth function $h_{c}: \mathcal{X}_{\mathrm{i}} \times \mathcal{B} \rightarrow$ $\mathbb{R}^{N_{a}}$, and a smooth function $\mathrm{v}: \mathcal{S} \rightarrow \mathcal{B}$ such that

$$
\begin{aligned}
& {\left[\begin{array}{c}
\dot{x} \\
\dot{\beta}
\end{array}\right]=\left[\begin{array}{c}
f(x)+g(x) u \\
0
\end{array}\right]=f_{e}\left(x_{e}\right)+g_{e}(x) u \quad x^{-} \notin \mathcal{S}} \\
& {\left[\begin{array}{l}
x^{+} \\
\beta^{+}
\end{array}\right]=\left[\begin{array}{l}
\Delta\left(x^{-}\right) \\
\mathrm{v}\left(x^{-}\right)
\end{array}\right]=\Delta_{e}\left(x_{e}\right) \quad x^{-} \in \mathcal{S},} \\
& y_{e}=h_{e}\left(x_{e}\right)=h(x)+h_{c}(x, \beta),
\end{aligned}
$$

with $h$ as defined in (55), satisfies the following properties:

(1) $h_{c}(x, 0) \equiv 0$ all $x \in \mathcal{X}_{\mathrm{i}}$;

(2) $\mathrm{v}\left(x^{*}\right)=0$ where $x^{*}=\overline{\mathcal{O}} \cap \mathcal{S}$ is the fixed point;

(3) $h_{e}$ has the same vector relative degree as $h$ (in this case two) on $\mathcal{X}_{e}=\check{\mathcal{X}}_{\mathrm{i}} \times \mathcal{B}$, where $\check{\mathcal{X}}_{\mathrm{i}}$ is an open neighborhood of $\mathcal{O} \cap \mathcal{X}_{\mathrm{i}}$;

(4) $h_{e}\left(x^{+}, \beta^{+}\right)=0$ and $L_{f_{e}} h_{e}\left(x^{+}, \beta^{+}\right)=0$ for all $x^{-} \in$ $\mathcal{S}, x^{+}=\Delta\left(x^{-}\right)$and $\beta^{+}=\mathrm{v}\left(x^{-}\right)$;

(5) there exists an open neighborhood $\mathcal{S}$ of $\mathcal{S}$ such that, for all $\beta \in \mathcal{B}$ and $x \in \breve{\mathcal{S}}, h_{c}(x, \beta)=0$.

System (64) is called a deadbeat hybrid extension. Properties (1) and (2) imply that the set $\mathcal{O}_{e}=\mathcal{O} \times\{0\} \subset \mathcal{X}_{e}$, called the trivial lift of $\mathcal{O}$, is a periodic orbit of (64). Property (3) implies the existence of a (new) zero dynamics manifold and associated restriction dynamics. Property (4) implies that the new zero dynamics manifold is hybrid invariant and contains $\mathcal{O}_{e}$. Property (5) implies that near $\mathcal{S}$, the projection of the new zero dynamics manifold onto $\mathcal{X}_{\mathrm{i}}$ equals the original zero dynamics manifold, $\mathcal{Z}_{\mathrm{i}}$.

Theorem 16 Morris and Grizzle [2009] (Hyrbid Invariance and Orbital Stability) The system (64) in closed-loop with the feedback controller defined on $\mathcal{X}_{e}$

$$
\begin{aligned}
u & =u_{e}^{*}-\left[L_{g_{e}} L_{f_{e}} h_{e}\right]^{-1}\left(\frac{1}{\epsilon^{2}} K_{p} h_{e}+\frac{1}{\epsilon} K_{d} L_{f_{e}} h_{e}\right) \\
u_{e}^{*} & =-\left[L_{g_{e}} L_{f_{e}} h_{e}\right]^{-1} L_{f_{e}}^{2} h_{e},
\end{aligned}
$$

with $K_{p}>0, K_{d}>0$, and $\epsilon>0$, satisfies the following properties:

(1) the manifold

$$
\mathcal{Z}_{e}=\left\{x_{e} \in \mathcal{X}_{e} \mid h_{e}\left(x_{e}\right)=0, L_{f_{e}} h_{e}\left(x_{e}\right)=0\right\}
$$

is hybrid invariant;

(2) $\mathcal{O}_{e}$ is a solution of the hybrid zero dynamics;

(3) $(\mathcal{S} \times \mathcal{B}) \cap \mathcal{Z}_{e}=\left(\mathcal{S} \cap \check{\mathcal{Z}}_{\mathrm{i}}\right) \times \mathcal{B}$, where $\check{\mathcal{Z}}_{\mathrm{i}}=\mathcal{Z}_{\mathrm{i}} \cap \check{\mathcal{X}}_{\mathrm{i}}$;

(4) the Poincaré return map for the hybrid zero dynamics $\left.P_{e}^{\epsilon}\right|_{\mathcal{Z}_{e}}:\left(\mathcal{S} \cap \check{\mathcal{Z}}_{\mathrm{i}}\right) \times \mathcal{B} \rightarrow\left(\mathcal{S} \cap \check{\mathcal{Z}}_{\mathrm{i}}\right) \times \mathcal{B}$ has the form

$$
P_{e}^{\epsilon} \mid \mathcal{Z}_{e}(z, \beta)=\left(\rho_{e}^{\epsilon}(z), \mathrm{v}(z)\right),
$$

where $\mathrm{v}$ is the parameter update law of the deadbeat hybrid extension and $\rho_{e}^{\epsilon}: \mathcal{S} \cap \check{\mathcal{Z}}_{\mathrm{i}} \rightarrow \mathcal{S} \cap \check{\mathcal{Z}}_{\mathrm{i}}$; and

(5) there exists $\bar{\epsilon}>0$ such that, for $0<\epsilon<\bar{\epsilon}$, the following are equivalent:

(a) $\mathcal{O}_{e}$ is an exponentially stable periodic orbit

(b) the original fixed point $x^{*}=\overline{\mathcal{O}} \cap \mathcal{S}$ is an exponentially stable fixed point of $\rho_{e}^{\epsilon}$.

\subsection{Gait Design}

The analytical results of Sections 5.1, 5.2, and 5.3 are rendered useful in feedback synthesis by introducing, in each domain $\mathcal{X}_{\mathrm{i}}$ of a biped model, a finite parametrization of the virtual constraints in (55) per

$$
h_{\alpha}(q):=h_{0}(q)-h_{d}(\theta, \alpha)
$$

in concrete applications, we have found it convenient to construct the function $h_{d}$ from Bézier polynomials; see Bézier [1972]. The parameters appearing in (68) introduce free parameters $\alpha$ into the zero dynamics of each domain,

$$
\dot{z}=f_{\text {zero }, \alpha}(z) \text {. }
$$

A fixed order of cycling the phases is postulated: $1 \rightarrow$ $2 \rightarrow \cdots \rightarrow N_{p} \rightarrow 1$. The search for a periodic walking motion is cast as a constrained nonlinear optimization problem: find parameters which minimize the integralsquared torque per step length ${ }^{26}$,

$$
J=\frac{1}{\text { step length }} \int_{0}^{T}\|u(t)\|_{2}^{2} d t
$$

\footnotetext{
${ }^{26}$ Torque being proportional to current in a DC motor, integral-squared torque is a rough approximation of energy dissipated in the motors.
} 
where $T$ is the total walking period through the $N_{p}$ phases. The optimization is carried out subject to natural constraints such as the following:

\section{inequality constraints}

- $\theta$ is strictly increasing (i.e, $\dot{\theta}>0$ along the solution of each domain);

- the solution respects the domain of admissibility, (31);

- positive vertical reaction force on the stance foot (a no-take-off constraint) (25);

- a friction constraint (27);

- bounds on allowed actuator torques;

- the swing foot is positioned above the ground in appropriate phases, etc.;

\section{equality constraints}

- conditions at the domain transitions that impose periodicity;

- desired walking speed;

- etc.

The parameters in the optimization include the coefficients in the virtual constraints, initial conditions of the model in the first phase, time spent in each phase, etc. The optimization problem is non-convex in the parameter set, with many local minima, and is very hard to solve. Nevertheless, fmincon in MATLAB and experience gained from studying simple planar robots in Westervelt et al. [2003] have so far allowed approximate solutions to be found for an interesting range of models.

For the purpose of finding a periodic orbit, the zero dynamics is used in each phase. This speeds up the integration of the various mechanical models. For phase-i, the input in (70) is evaluated as

$$
u_{\alpha, \mathrm{i}}^{*}:=-\left.\left[L_{g} L_{f} h_{\alpha}\right]^{-1} L_{f}^{2} h_{\alpha}\right|_{\mathcal{Z}_{\mathrm{i}}},
$$

assuming the virtual constraints have vector relative degree two. Once a periodic orbit is found, a hybrid zero dynamics feedback controller is synthesized via Sect. 5.3, and the stability of the closed-loop hybrid model is evaluated via a Poincaré map as in Theorem 16. The feedback controller is not necessarily synthesized with the same virtual constraints used to find the periodic orbit, though it often is; for details, see Westervelt et al. [2007a] and Chevallereau et al. [2009b].

Remark 17 The important role of the optimization criterion in gait design has been extensively explored; see Srinivasan and Ruina [2006] and references therein. The design of virtual constraints on the basis of the dimensionless cost of mechanical transport is performed in [Sreenath et al., 2011b, Eq. (40)] and [Ramezani et al., 2014, Eq. (17)]. As an alternative to optimization, one could imagine choosing virtual constraints so that the resulting zero dynamics is diffeomorphic to a specified target dynamics, as in the templates and anchor framework of Full and Koditschek [1999]. To date, this has been achieved in only one case; see Poulakakis and Grizzle [2009].

Remark 18 The feedback (59) is inverting the dynamics associated with the virtual constraints. Inversion or "cancelling the natural dynamics" is generally believed to be a bad thing. Does this make the whole method suspect? First of all, experimental implementation of the method is discussed in Westervelt et al. [2004], Sreenath et al. [2011b], Sreenath et al. [2011a], and Park et al. [2013]. $A$ key point is that when the virtual constraints are designed to minimize a cost function such as (70), they tend to use low control effort which means that the "natural dynamics" are used as much as possible instead of being cancelled. This aspect is made especially clear in Westervelt et al. [2007b], where an actuated compass gait biped is controlled using virtual constraints and zero dynamics in such a manner that the controller uses zero torque when walking down a slope on the periodic orbit of the unactuated biped, and only expends control effort to return to the periodic orbit. A second point is that in practice, exponential stability is robust to sufficiently small perturbations, and thus approximately zeroing the virtual constraints tends to result in good experimental behavior. A third point is that input-output linearization can be replaced with other types of feedback, as discussed in Sect. 6.4 and in Galloway et al. [2013].

\subsection{Illustration on an Underactuated Biped}

The design of a stabilizing controller for a simple 3D bipedal robot is illustrated here with simulation results $^{27}$. The influence of the frontal (coronal) plane dynamics on the overall motion of the robot will be emphasized as this is the major new element when passing from $2 \mathrm{D}$ to $3 \mathrm{D}$. With this in mind, the simplest mechanical structure that highlights this aspect of the gait design and stabilization problem will be used.

Biped: The 3D-biped depicted in Fig. 7 is taken from Chevallereau et al. [2009b]. It consists of five links: a torso and two legs with revolute one DOF knees that are independently actuated and terminated with "pointfeet". Each hip consists of a revolute joint with two DOF and each DOF is independently actuated. The width of the hips is nonzero. The stance leg is assumed to act as a passive pivot in the sagittal and frontal planes, with no rotation about the z-axis (i.e., no yaw motion). Indeed, the small link in the diagram that appears to form a foot has zero length and no mass. Its purpose is to indicate the two DOF at the leg-ground contact point corresponding to motion in the frontal $\left(q_{1}\right)$ and sagittal $\left(q_{2}\right)$

\footnotetext{
${ }^{27}$ Recent experiments can be found in Grizzle [2014a] and Grizzle [2014b]
} 
planes; in addition, it shows that there is no yaw rotation about the stance leg end per Sect. 3.4 and Fig. 4. The angles $q_{1}$ and $q_{2}$ are unactuated. The remaining joints are independently actuated. In single support, the robot is underactuated, having $8 \mathrm{DOF}$ and 6 independent actuators. The physical parameters of the robot are given in Table 1.

Studied Gait: The walking gait consists of phases of single support, alternating on the left and right legs, with transitions determined by the height of the swing leg above the ground becoming zero. The impact of the swing leg with the ground is assumed to be rigid as in Sect. 3.6, and, consequently, the double support phase is instantaneous. Following Sect. 3.4, a dynamic model is easily developed. The hybrid model naturally has two continuous domains, corresponding to single support on the left and right legs, respectively. If a gait is sought that is symmetric with respect to the left and right legs, it is possible (and common) to use a model with a single continuous phase, and to "flip" the sign of the hip width from one step to the next; see Chevallereau et al. [2009b].

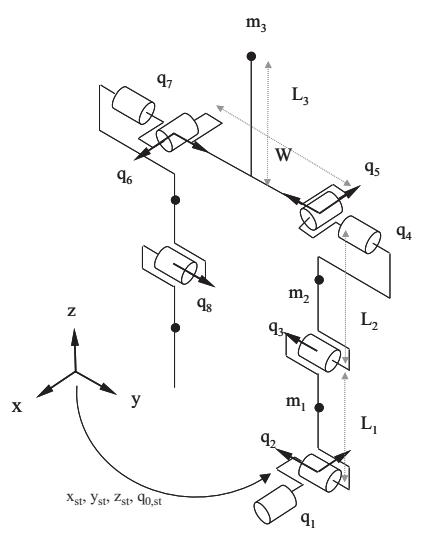

(a)

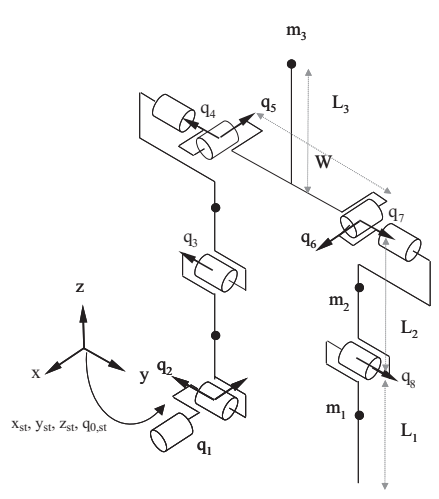

(b)
Fig. 7. A five-link 3D biped with point feet that is $0.55 \mathrm{~m}$ at the hip and has a total mass of $7.25 \mathrm{Kg}$. (a) shows the coordinates for single support on leg-1 and (b) shows the coordinates for single support on leg- 2 .

\begin{tabular}{|l|l|l|l|l|l|l|l|}
\hline $\mathrm{g}$ & $\mathrm{W}$ & $\mathrm{L} 1$ & $\mathrm{~L} 2$ & $\mathrm{~L} 3$ & $\mathrm{~m} 1$ & $\mathrm{~m} 2$ & $\mathrm{~m} 3$ \\
\hline 9.81 & 0.15 & 0.275 & 0.275 & 0.05 & 0.875 & 0.875 & 5.5 \\
\hline
\end{tabular}

Table 1

Parameters for the 3D bipedal robot (in MKS).

Periodic Orbit: A symmetric, periodic walking gait was found as in Sect. 5.4. The model has two continuous phases, corresponding to support on leg-1 and then leg2 . In each phase, the function $h_{0}(q)$ in (55) was selected as the actuated variables, namely,

$$
h_{0}(q)=\left[\begin{array}{c}
q_{3} \\
\vdots \\
q_{8}
\end{array}\right] \text {. }
$$

The variable $\theta$ was taken as

$$
\theta=-q_{2}-q_{3} / 2
$$

which corresponds to the sagittal plane angle of the line connecting the leg end to the hip. Bezier polynomials of degree 3 were chosen for $h_{d}(\theta)$ to complete the definition of the virtual constraints. The parameters were then selected by seeking a (local) minimum of (70).

The computed gait has an average walking speed of 0.75 leg lengths per second; the step length is 0.32 leg lengths; and the step width is close to the hip width. The nominal gait's joint profiles over two consecutive steps are shown in Fig. 8. The unactuated and actuated variables are presented; note that $\theta$ is monotonic over each step. Fig. 9 shows the torque required to produce the periodic motion. Fig. 10 shows the profile of the ground reaction force on the stance foot and the profile of the swing leg end; this figure shows that the unilateral contact constraints are satisfied on the nominal periodic orbit.
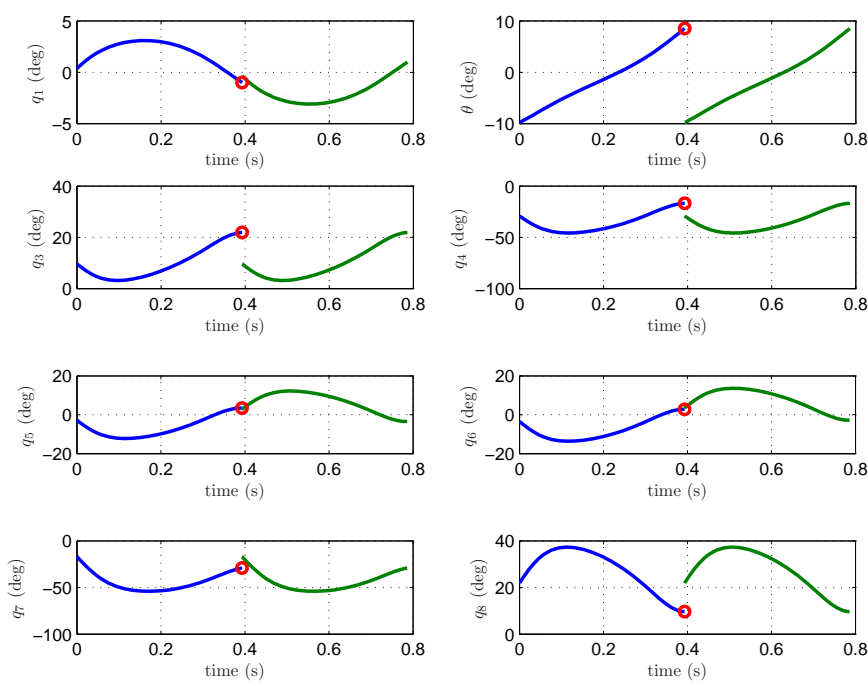

Fig. 8. Joint profiles of a periodic motion that is symmetric over two steps. The small circles represent the points where the discrete transitions occur. Plots of $q_{k}$ versus $\theta$, for $3 \leq k \leq 8$ define the virtual constraints.

Feedback Control: The periodic orbit essentially came with a set of nominal virtual constraints based on the controlled variables (72). Using outputs based on these constraints, a hybrid zero dynamics feedback controller was synthesized following the method of Sect. 5.3. The stability of the closed-loop hybrid model was evaluated via the restricted Poincaré map as in Theorems 8 and 16. The eigenvalues of the Jacobian of the restricted Poincaré map evaluated at the fixed point were

$$
\left|\lambda_{1}\right|=0.89, \quad\left|\lambda_{2}\right|=0.70, \quad\left|\lambda_{3}\right|=2.1
$$

One eigenvalue has magnitude greater than one and hence the gait is unstable under this controller. 

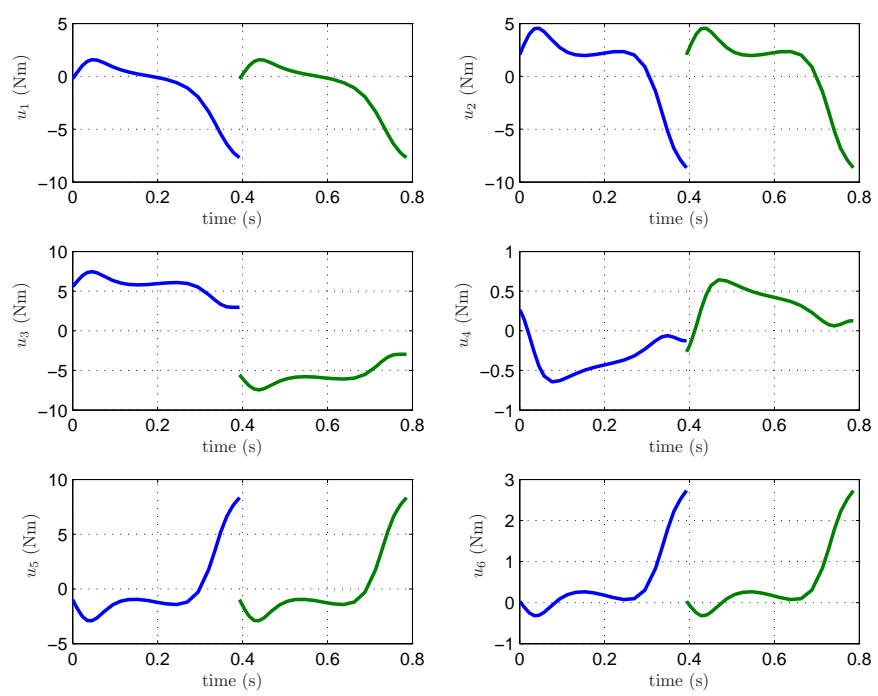

Fig. 9. Torques profiles of the periodic motion over two steps.
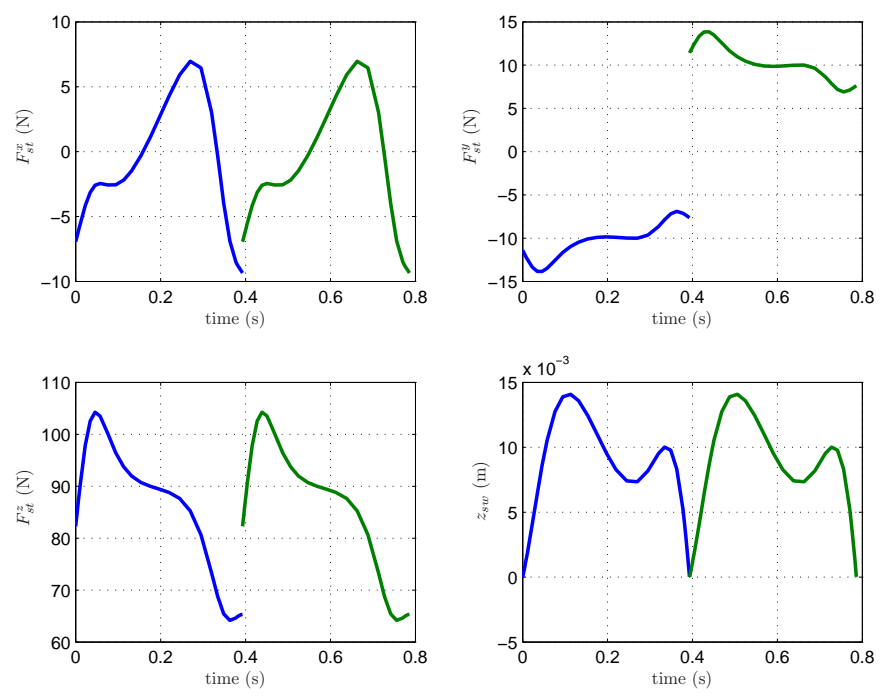

Fig. 10. The reaction forces on the stance foot over two steps and the evolution of the swing leg end. The nominal orbit satisfies the required unilateral constraints at ground contact, as specified in (41).

An analysis of the eigenvectors did not clearly associate a particular "component" of the state of the HZD with the unstable eigenvalue. Based on results in [Westervelt et al., 2007a, pp. 160-163], however, the sagittal plane motion was expected to be stable, so it was suspected that the instability arose in the frontal plane motion. Indeed, the position of the center of mass in the frontal direction is important. If, at leg touchdown, the center of mass is not between the feet, but outside the position of the next supporting foot, the robot will topple sideways. Based on this physical intuition, the control of the variable $q_{6}$ (which regulates step width on the swing leg) was replaced by the control of the distance between the swing leg end and the center of mass along the frontal direction; denote this distance by $d(q)$.
To incorporate this new objective into the control law, the fourth component of the function $h_{0}(q)$ in the virtual constraints $(72)$ was replaced with ${ }^{28} d(q)$. On the nominal periodic orbit, this distance was evaluated and approximated by a Bezier polynomial in $\theta$, denoted $d^{*}(\theta)$. When the HZD control law of Sect. 5.3 was recomputed using this new output, the walking gait was stable, as shown via the calculation of the eigenvalues of the linearization of the restricted Poincaré map:

$$
\left|\lambda_{1}\right|=0.78, \quad\left|\lambda_{2}\right|=\left|\lambda_{3}\right|=0.25
$$

Remarks on 2D vs. 3D: When extending the method of virtual constraints from planar robots with one degree of underactuation to $3 \mathrm{D}$ robots with two or more degrees of underactuation, new challenges and properties appeared. In the 2D case, hybrid invariance could be achieved without use of a deadbeat hybrid extension. Indeed, Theorem 5.2 in Westervelt et al. [2007a] shows that, under very mild conditions, hybrid invariance follows from the nominal virtual constraint vanishing along a periodic orbit. Moreover, the restricted Poincaré map is scalar, can be computed in closed form, and an analytical condition for asymptotic orbital stability can be obtained, based on physical properties of gait along the periodic orbit, and independent of the virtual constraints used to parameterize the orbit. For a robot evolving in $3 \mathrm{D}$, with two or more degrees of underactuation, creating hybrid invariant manifolds is much more challenging. The only known method to achieve invariance under the rest maps is to use a deadbeat hybrid extension. The resulting restricted Poincaré map is of dimension three or higher, and conditions for asymptotic orbital stability depend on the particular choice of the virtual constraints used to parametrize the orbit, as was illustrated here.

\section{Advanced Aspects of Control via Virtual Con- straints}

This sections points to recent advances in the theory of virtual constraints and hybrid zero dynamics. The development is deliberately less detailed than in Sect. 5 . The primary objective is to indicate recent trends.

\subsection{Non-Trivial Feet}

When a robot has nontrivial feet, the point-foot control strategy presented in Sect. 5 can be applied without difficulties if the following additional issues are taken into account:

- The gait can be more complex. It can have several phases corresponding to different kinds of contact between the foot and the ground. This point is illustrated for example in Fig. 12.

\footnotetext{
${ }^{28}$ A linear approximation was in fact used.
} 

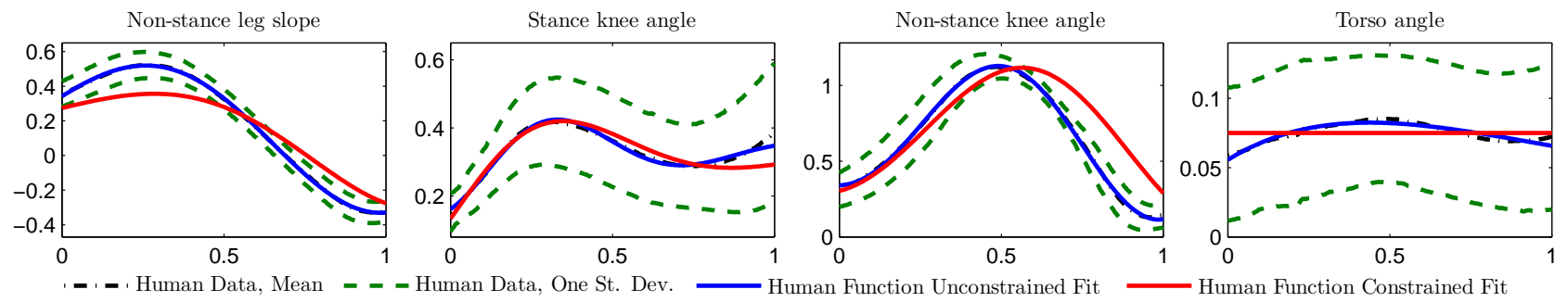

Fig. 11. The canonical walking function (74) parallels many human-inspired outputs like the four common ones featured in this figure. The human data here represent an amalgamation of experimental data collected over numerous trials from nine test subjects as detailed in Sinnet and Ames [2012a]; in the literature, e.g., in Perry and Burnfield [2010], healthy walking is defined as being within one standard deviation of the mean. The two human data fits depicted with solid lines represent both unconstrained fits of (74) to human walking data and fits constrained to satisfy the conditions of partial hybrid zero dynamics on the robot AMBER 1 (see Kolathaya et al. [2012] and Yadukumar et al. [2013]), i.e., these fits result in stable walking. The unconstrained fits tend to have correlations within one percent of unity justifying the choice of (74).

- When the stance foot is flat on the ground, the system is fully actuated and the control must ensure that the constraints collected in (29) are satisfied. Among these, the constraint (28) is the most difficult to satisfy, and several strategies have been developed to achieve this objective Hirai et al. [1998], Kajita et al. [2003].

- The evolution of the swing foot must be managed so that the landing of the swing foot occurs in an appropriate way.

Wang et al. [2012, 2014] presented an extension of the underactuated control approach to fully actuated robots with feet by directly regulating the evolution of the ZMP. For a robot with point feet, the center of pressure $(\mathrm{CoP})$ is located at the point of contact, and thus the robot's motion automatically has the ZMP at the point of contact. In the case of a robot with a foot in flat contact with the ground, the path of the ZMP was moved into the control objectives, defined as a function of $\theta$, just as was done previously for the joint variables. Thus the set of virtual constraints or outputs was augmented with $y_{Z M P}=Z M P_{0}-Z M P^{d}(\theta)$.

Because the evolution of the ZMP is controlled, phases of walking with rotation about the toe or the heel, as described in Sect. 3.3, can be treated using the same control strategy. Even in the case of a fully actuated humanoid, as long as two outputs have been defined to ensure the satisfaction of the constraint on the ZMP, the system can be view as an underactuated system of relative degree 2, as treated in Sect. 5. Thus the same approach can be used. In case of a nontrivial foot, the absolute orientation of the swing foot must be controlled rather than the relative orientation of the swing ankle to achieve an appropriate landing configuration in the eventual presence of error.

The proposed method can be viewed as an on-line modification of the reference trajectory in order to ensure the satisfaction of the contact constraint. The main point is

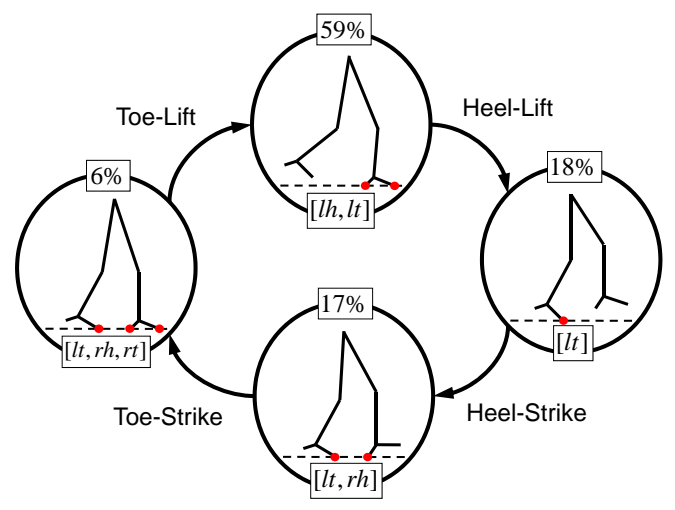

Fig. 12. An example of a domain breakdown, i.e., the discrete phases of a walking gait, based upon a specific temporal ordering of contact points. The red dots indicate the constraints enforced in each discrete phase (or domain). The percentages shown for each domain indicate the approximate amount of the gait which humans spend in each domain; in particular, note that approximately $20 \%$ of the gait is spent in double support. In addition, the gait contains periods of full, under and over actuation.

that the effect of this on-line modification on the stability of a gait is studied on the basis of a rigorous stability analysis, and not by hand tuning in experiments. More importantly, the stability during the foot rotation phase can also be taken into account, something that has been missing in previous studies for walking gaits with foot rotation.

\subsection{Human-Inspired Output Functions}

Motivated by the biomechanics literature, ${ }^{29}$ humanwalking can be used to inspire the construction of hybrid system models, along with virtual constraints - inspired by human locomotion data - that yield provably stable walking gaits.

\footnotetext{
29 The study of human kinematics is common for biomechanics researchers; see, e.g., Winter [2009].
} 
In the context of hybrid system models, as illustrated in Sect. 3, a hybrid system model of a walking gait is essentially completely determined by the contact points over the course of a step; this is explained in further detail, using the modelling methodologies of this paper, in Ames et al. [2011]. This motivates the use of human dataspecifically, the temporal ordering of contact points - in order to determine the discrete domains of a hybrid system model associated with a periodic walking gait. Utilizing human motion capture experiments, Ames et al. [2011] and Vasudevan et al. [2013] determined a metric for comparing different walking gaits by constructing weighted graphs associated with each gait and employing a variant of the cut distance between graphs. Through these constructions, it was found that all healthy subjects displayed the same universal domain breakdown composed of four discrete phases (shown in Fig. 12). In addition, utilizing the optimal walking cycle obtained by finding the graph with minimum distance between the graphs associated with the walking gait of each subject, an initial metric for gauging the human-like nature of a walking gait was proposed. These results provide evidence for the importance of multi-domain walking gaits in achieving human-like locomotion.

Studies such as Kirtley et al. [1985] have shown through gait analysis that numerous parameters of gaits do not seem to be affected by a person's height or weight and such results motivate a kinematics-based gait design approach. In the context of controller synthesis as motivated by human locomotion data, the goal is to utilize kinematic walking data to construct virtual constraints as outlined in Sect. 5. With this goal in mind, consider a human output combination (see Ames [2014]): $Y^{H}=\left(Q, y_{1}^{H}, y_{2}^{H}\right)$, consisting of the configuration space of a robot, $Q \subset \mathbb{R}^{n}$, a velocity modulating output $y_{1}^{H}$ : $Q \rightarrow \mathbb{R}$, position modulating outputs $y_{2}^{H}: Q \rightarrow \mathbb{R}^{n-1}$ given by $y_{2}^{H}(q)=\left[y_{2}^{H}(q)_{i}\right]_{i \in O}$ with $O$ an indexing set for $y_{2}^{H}$. By considering human walking data, specific human output combinations appear to kinematically characterize human walking through the canonical walking function $(C W F)$ :

$$
y_{H}(t, \alpha)=e^{-\alpha_{4} t}\left(\alpha_{1} \cos \left(\alpha_{2} t\right)+\alpha_{3} \sin \left(\alpha_{2} t\right)\right)+\alpha_{5} \text {. }
$$

In particular, consider human data taken over one step of a walking gait at discrete times $t[k], k \in\{1, \ldots, N\}$ yielding discrete angle measurements of the human $q^{H}[k] \in Q$ as appropriately mapped to the robot model. For the proper human output combination and choice of parameters, $\alpha=\left(v,\left(\alpha_{i}\right)_{i \in O}\right)$, it appears that (see Fig. 11):

$$
y_{1}^{H}\left(q^{H}[k]\right) \approx v t^{H}[k], \quad y_{2}^{H}\left(q^{H}[k]\right) \approx\left[y_{H}\left(t^{H}[k], \alpha_{i}\right)\right]_{i \in O} .
$$

This implies that a proper human output combination characterizes the behavior of human walking at a kine- matic level to be that of a linear mass-spring-damper system. Specific examples of human output combinations have been studied in numerous papers, including Sinnet et al. [2011], Ames [2011] and Jiang et al. [2012]. For example, the forward position of the hip or forward position of the center of mass can be taken to be the velocity modulating output, and 4 examples of position modulating outputs are shown in Fig. 11.

With the goal of driving outputs of the robot to outputs of the human, as expressed by (74), human output combinations can be used to synthesize virtual constraints (see Sect. 5) by considering the following human-inspired outputs consisting of relative one and vector relative degree two outputs ${ }^{30}$ of the form:

$$
\begin{aligned}
y_{1}(q, \dot{q}) & =d y_{1}^{H}(q) \dot{q}-v \\
y_{2}(q) & =y_{2}^{H}(q)-\left[y_{H}\left(\theta(q), \alpha_{i}\right)\right]_{i \in O} .
\end{aligned}
$$

where $\theta(q)$ is a parameterization of time based upon the velocity modulating output $y_{1}^{H}$ :

$$
\theta(q)=\frac{y_{1}^{H}(q)-y_{1}^{H}\left(q^{+}\right)}{v},
$$

with $q^{+}$the system configuration at the beginning of a step, and $v$ is the desired velocity of the velocity modulating output (76). This choice of parameterization is motivated by the fact that velocity modulating outputs are chosen by the critera that they are expressed by a linear function of time (75). Utilizing the human-inspired outputs, the feedback linearizing controller (59) can be adapted to drive $h:=y_{2} \rightarrow 0$ in the case of underactuation, and a slight modification can be used to drive $y_{1} \rightarrow 0$ and $y_{2} \rightarrow 0$ in the case of full actuation. Intuitively, driving these outputs to zero drives the outputs of the robot - both velocity and position modulating to the outputs of a human as represented by a constant for the velocity modulating output and the CWF for the position-based outputs.

The parameters, $\alpha$, of the CWF that best fit the human data will not generally result in robotic walking due to differences between the robot and human. Although it is possible to obtain walking through the heuristic procedure of simply fitting (74) to human data and manually tuning parameters as was done in Sinnet et al. [2011], a more effective approach involves performing the fitting subject to conditions on hybrid invariance and using the framework of virtual constraints to achieve formal stability (see Ames [2014] for the full development of these ideas in the case of full and under actuation). We will briefly outline this construction in the context of full actuation for a robot with $n$ degrees of actuation. The

\footnotetext{
${ }^{30}$ Note that the human output combination must be properly chosen to ensure proper relative degree, i.e., invertibility of the decoupling matrix.
} 
feedback controller utilizing the human-inspired outputs renders the zero dynamics manifold (58) forward invariant and attractive for the continuous dynamics. Since we are assuming full-actuation, and this manifold is defined in terms of only the relative degree 2 outputs we term it the partial zero dynamics manifold and denote it by $\mathcal{P} \mathcal{Z}_{i}$. The human-inspired optimization problem is given by:

$$
\begin{aligned}
\left(v^{*}, \alpha^{*}\right)=\underset{(v, \alpha) \in \mathbb{R}^{5(n-1)+1}}{\operatorname{argmin}} \operatorname{Cost}_{\mathrm{HD}}(\alpha) \\
\text { s.t. } \Delta\left(\mathcal{P} \mathcal{Z}_{\mathrm{i}} \cap \mathcal{S}\right) \subset \mathcal{P} \mathcal{Z}_{\mathrm{i}}
\end{aligned}
$$

where Cost $_{\mathrm{HD}}: \mathbb{R}^{(5 n-1)} \rightarrow \mathbb{R}$ is the least squares fit of the CWF to the experimental human walking data over one step. The novelty of human-inspired optimization (Ames [2011]) lies in the specific formulation of the constraints $\Delta\left(\mathcal{P} \mathcal{Z}_{\mathrm{i}} \cap \mathcal{S}\right) \subset \mathcal{P} \mathcal{Z}_{\mathrm{i}}$ as they can be expressed in terms of only the parameters $\left(v^{*}, \alpha^{*}\right)$. Moreover, additional constraints that ensure the physical validity of the resulting walking gait, e.g., torque bounds, velocity bounds, and ZMP constraints (28), can be added to the optimization problem and, again, be expressed in terms of only the parameters. The parameters solving the human-inspired optimization problem, $\left(v^{*}, \alpha^{*}\right)$, provably result in a stable walking gait for which a fixed point can be found explicitly (Ames [2014]). These methods have been successfully applied experimentally to achieve walking on 3D bipedal robots Ames et al. [2012a], Powell et al. [2013], Dantam et al. [2013]. In addition, the application of these methods to underactuated robots ${ }^{31}$ was considered in Ames [2012] and the results have been demonstrated in practice in Kolathaya et al. [2012], Yadukumar et al. [2013].

\subsection{Series-Compliant Actuation}

Inspired by Raibert's hoppers (Raibert [1984], Raibert and H. Brown, Jr. [1984], Raibert [1986]), and also by spring flamingo and spring turkey (Hollerbach et al. [1992], Pratt and Pratt [1999], Pratt [2000], Pratt et al. [2001]), Hurst designed the planar bipedal robot MABEL and the 3D bipedal robot ATRIAS to include large springs, as shown in Fig. 13. In the case of MABEL, the springs are (roughly speaking) placed in series between the actuators and the knee joints Park et al. [2011]. In the case of ATRIAS, the springs are between the actuators and the top two links of the 4-bar mechanism forming the legs Grimes and Hurst [2012]. In each case, the springs serve to isolate the reflected rotor inertia of the motors from the impact forces at leg touchdown and to store energy in the compression phase of a running gait,

\footnotetext{
${ }^{31}$ The first use of human data in conjunction with virtual constraints in the context of underactuated robots can be found in Srinivasan et al. [2008, 2009]. In this case, methods based upon those presented in Sect. 5.4 were used.
}

when the support leg must decelerate the downward motion of the robot's center of mass; the energy stored in the spring can then be used to redirect the center of mass upwards for the subsequent flight phase, when both legs will be off the ground Alexander [1990], Chevallereau et al. [2005], Full and Koditschek [1999]. Both of these properties (shock isolation and energy storage) enhance the energy efficiency of locomotion. On the other hand, the springs increase the number of degrees of freedom in the robot, with no attendant increase in the number of actuators.

To date, the design of the zero dynamics for series elastic actuators has been addressed in two distinct ways. In Morris and Grizzle [2009], the spring model of Spong [1987] was adopted. It was shown that if the controlled variables $h_{0}(q)$ are taken on the joint side of the spring, the relative degree of those components of the output becomes 4 instead of 2 , and the zero dynamics of the robot with series elastic actuation is diffeomorphic to that of the robot without series elastic actuation. In particular, the dimension of the zero dynamics does not increase, which favors simplicity of any attendant stability analysis. On the other hand, if the the controlled variables $h_{0}(q)$ are taken on the motor side of the springs, the relative degree remains 2 and the dimension of the zero dynamics increases; see for example Poulakakis and Grizzle [2009]. Which approach is better? Reference Poulakakis and Grizzle [2007] designed two controllers for a hopper model with compliance; one controller used virtual constraints with relative degree 2 outputs and the other controller used outputs with relative degree 4 . The same pe-

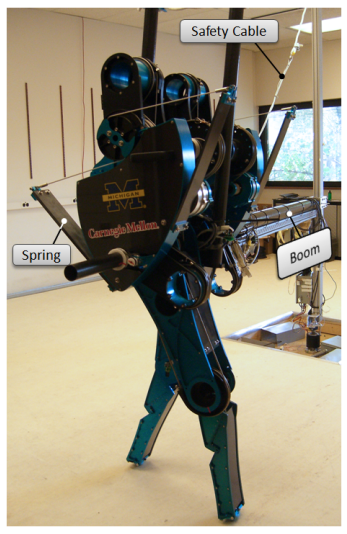

(a)

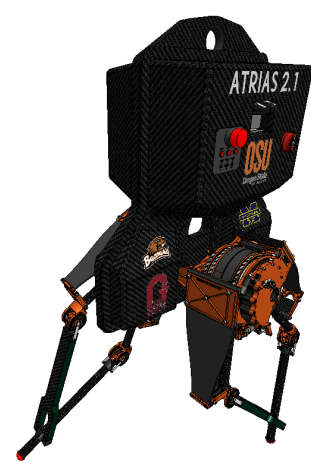

(b)
Fig. 13. (a) The planar (2D) biped MABEL is one meter tall at the hip and has overall mass of $65 \mathrm{Kg}$. Because the hips are 1 degree-of-freedom (DOF) revolute joints, the robot is tethered, walks in a cylindrical approximation to the sagittal plane, and cannot leave the laboratory. (b) 3D biped ATRIAS is one meter tall at the hip and has a total mass of $55 \mathrm{Kg}$. Each hip has 2 DOF, allowing the leg to move in the frontal and sagittal planes, and thus ATRIAS can balance itself side-to-side without the aid of a boom. ATRIAS is battery powered and is designed for both indoor and outdoor use. Both robots use large springs in their actuation. 
riodic orbit was realized with each controller, so that the robot in closed-loop had the same steady-state hopping motion in each case. The difference showed up in transient operation: the controller based on outputs with relative degree 2 used lower peak torques and consumed less actuator power when rejecting common disturbances. The feedback controller based on the relative degree 2 outputs is simpler to compute and implement than the controller based on relative degree 4 outputs. In simulations, it also seems to preserve stability for a wider range of uncertainty in the spring constant.

The robot MABEL has been controlled with relative degree 2 outputs and they have performed remarkably well in experiments Sreenath et al. [2011b, 2012], Park et al. [2012, 2013], allowing the robot to accommodate unseen stepdowns of $20 \mathrm{~cm}$ and to run on level ground at a peak speed of $3 \mathrm{~m} / \mathrm{s}$. Relative degree 2 outputs have performed well in simulations of the $3 \mathrm{D}$ robot ATRIAS Ramezani et al. [2014]. Experimental confirmation for 3D ATRIAS has just recently been demonstrated in Grizzle [2014b].

\subsection{Control Lyapunov Functions for Zeroing the Vir- tual Constraints}

In the control law (59), the term $u^{*}$ given by (60) renders the zero dynamics manifold forward invariant, while the term

$$
\left[L_{g} L_{f} h(x)\right]^{-1}\left(\frac{1}{\epsilon^{2}} K_{p} h(x)+\frac{1}{\epsilon} K_{d} L_{f} h(x)\right)
$$

accomplishes two things: it renders the input-output map linear and renders the zero dynamics manifold locally exponentially attractive, with the rate of convergence adjusted through the choice of $\epsilon$.

Theorem 16 clarifies the importance of being able to tune the rate of convergence because it states that when the "transverse variables" converge sufficiently rapidly to zero, exponential stability of a periodic orbit in the zero dynamics implies exponential stability of the orbit in the full model. Is the input-output linearization property somehow fundamental?

Ames et al. [2012b] shows that it is not at all necessary to linearize the input-output map; it is only the ability to tune the rate of exponential convergence that is important. Specifically, the notion of a control Lyapunov function (CLF) (Sontag [1989], Grognard and de Wit [2004], Hauser and Chung [1994]) is extended to the hybrid setting, and then refined to allow for the tuning of the rate of exponential convergence through the definition of a Rapidly Exponentially Stabilizing Control Lyapunov Function or RES-CLF for short. The point-wise minimum norm of Petersen and Barmish [1987], Freeman and Kokotović [1996] is extended to RES-CLFs and validated experimentally on MABEL in Ames et al.

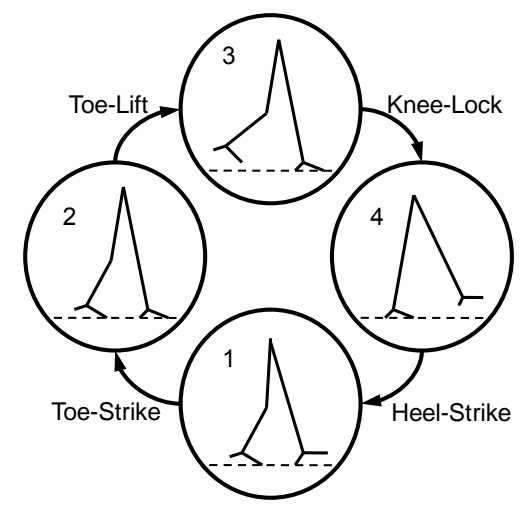

Fig. 14. Directed graph of proposed model.

[2012c]. Furthermore, actuator bounds can also be taken into account via a Quadratic Program (QP), as shown in Galloway et al. [2013]. More recent work can be found in Ames and Powell [2013], Morris et al. [2013].

\section{Controlled Routhian Reduction}

First introduced in Ames [2006], functional Routhian reduction has been helpful in achieving $3 \mathrm{D}$ walking in a number of bipeds in simulation (see, e.g., Ames et al. [2007, 2009], Sinnet and Ames [2009b]) and has also been demonstrated in practice in Sinnet and Ames [2012a,b]. By shaping the energy of a biped through control, functional Routhian reduction can effectively decouple the sagittal and coronal dynamics of a 3D biped and allow for control design to be conducted with the dynamics restricted to the sagittal plane. ${ }^{1}$ This decoupling allows one to harness existing methods for control of 2D bipeds. Moreover, as a consequence of the reduction procedure, the biped will be stabilized in an upright configuration in the coronal plane. Thus in addition to simplifying control design for 3D bipeds, functional Routhian reduction also points to the inherent decoupled nature of walking.

Beginning with a 3D biped, a sagittal restriction is applied to arrive at a reduced-order model operating in the sagittal plane. Controllers are then designed to achieve sagittal-plane walking on this simplified model. For the example herein, controlled symmetries will be used with additional spring-damper-like control laws which will provide an overall passivity-based feel. Once stable 2D walking has been found, energy shaping is used to transform the Lagrangian of the 3D biped into a form amenable to functional Routhian reduction. The reduced system obtained by stacking this form of reduction with sagittal control has the same dynamics as the 2D biped. Finally, the decoupling afforded by reduction can only be guaranteed on a manifold on which certain initial conditions of the continuous dynamics hold (as will be seen in Theorem thm:reduction) and thus feedback linearization is used to render this manifold exponentially stable. The end result is stable 3D 
bipedal walking which is identical to the $2 \mathrm{D}$ biped with respect to the continuous dynamics.

The rest of this section describes and illustrates functional Routhian reduction on a biped with feet, locking knees, and a hip, as in Sinnet and Ames [2009b]. Nontrivial anthropomorphic foot action obtained through control and a four-domain hybrid model is used to treat the different phases of walking shown in Fig. 14.

\subsection{D Model}

The model of interest in this section is composed of rigid links with point masses as shown in Fig. 16. The model will have configuration space $\mathcal{Q}_{3 \mathrm{D}}$, which will consist of the extended coordinates

$$
q_{e}=\left(p_{b}^{\prime}, \phi_{b}^{\prime}, q^{\prime}\right)^{\prime}
$$

where $p_{b}$ and $\phi_{b}$ are, respectively, the position and Euler angles for the orientation of a point on the torso. The joint angles for the stance leg are the same as angles $q_{1}, \ldots, q_{4}$ shown in Fig. 4 . The numbering of the angles $q_{5}, \ldots, q_{8}$ begins at the hip and each angle twists about the opposite axis of its counterpart on the stance leg in the same manner as shown in Fig. 7. A gait can be constructed for this model based on the domain graph shown in Fig. 14. The nodes on the graph are dictated by those constraints which are enforced by the environment such as ground contact and knee lock (assuming mechanical locks).

The validity of the desired domain graph depends on the choice of control; certain controllers may cause the foot contact to enter into undesirable states (which are not present in Fig. 14.) The system itself can be modeled as the following hybrid control system (as in (53)):

$$
\Sigma_{3 \mathrm{D}}=\left(\Gamma_{3 \mathrm{D}}, \mathcal{X}_{3 \mathrm{D}}, \mathcal{U}_{3 \mathrm{D}}, \mathcal{S}_{3 \mathrm{D}}, \Delta_{3 \mathrm{D}}, \mathcal{F} \mathcal{G}_{3 \mathrm{D}}\right)
$$

where $\Gamma_{3 \mathrm{D}}$ is an oriented graph as pictured in Fig. 14 and contains domain-specific information regarding which points are in contact with the environment. Each of the symbols in the tuple above is an array of elements such as $\mathcal{X}_{3 \mathrm{D}}=\left\{\mathcal{X}_{3 \mathrm{D}}^{i}\right\}_{i=1}^{4}$ containing different elements for different phases of walking. Each domain will be described in deatil and examples will be given on how to construct specific elements for the tuple (80) and the reader may feel free to extrapolate to determine those elements which have been omitted for the sake of clarity. Before delving into the individual domains, however, a discussion of some general considerations is pertinent.

General Considerations. Inherent symmetries in bipedal walking allow one to simplify a gait's description by considering the system in terms of stance-swing leg rather than left-right leg: a discrete state variable

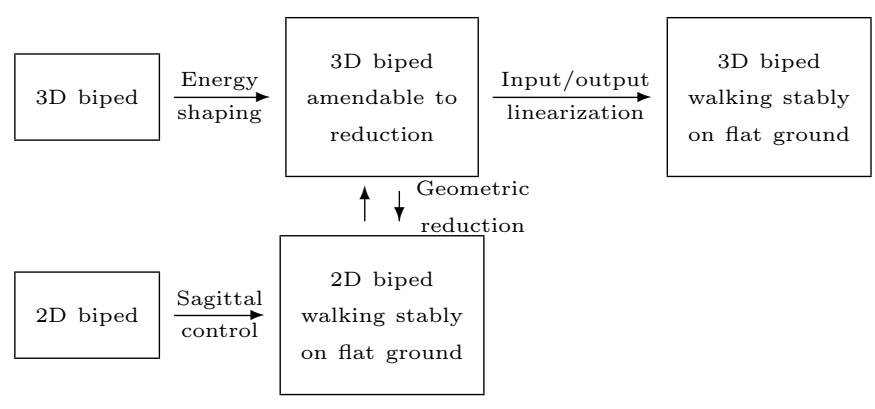

Fig. 15. Proposed scheme for obtaining walking.

can be used to track which leg is currently the stance leg but one is free to examine the system over a single step rather than an entire step cycle thereby simplifying analysis.

By convention, the phases are numbered such that the transition from the last domain to the first domain (i.e., $4 \rightarrow 1$ ) corresponds to heel strike as this event signifies that the stance and swing legs should be swapped. For the proper choice of gains, the walking controllers applied in this example can generate a gait for which the hybrid dynamics $h_{1}\left(q_{e}\right)=p_{s t t}^{z}\left(q_{e}\right)$, where $p_{s t t}^{z}\left(q_{e}\right)$ is height of the stance toe above the ground, can be combined to construct the constraint vector $H_{1}\left(q_{e}, \dot{q}_{e}, u\right)$. The unilateral constraint provides a metric for determining the distance of the system from the edge of the domain; when this metric reaches zero, the discrete dynamics of plastic impacts comes into play, operating on the pre-impact state to provide the post-impact state as an initial condition for the continuous dynamics of the next domain.

After impact, it is desired that the stance knee be locked, that the stance foot be flat on the ground, and that the swing toe remain fixed to the ground. These requirements dictate an apropos choice of kinematic constraints for constructing a Jacobian for the impact map (47). Using toe strike as the transition leads to the switching surface $\mathcal{S}_{3 \mathrm{D}}^{1 \rightarrow 2}$ given in (52). As alluded to, the impact should be treated in exactly the same manner as the continuous dynamics. Specifically, the friction, ZMP, and normal force constraints must be checked. However, additional checks must be performed to ensure no point has a post-impact trajectory into the ground.

Phase 2-Toe Lift $(t l)$. As the stance foot experiences heel roll from the previous phase and the toe rolls into the ground causing an impact, the stance foot enters a state of flat foot contact while the swing toe remains on the ground. The system continues under these conditions until the vertical constraining force on the back (swing) toe reaches zero, at which point, the ground is no longer undergoing a force interaction with the toe. 
This force thus represents a holonomic constraint on the system which can be used to define both the switching surface and domain of admissibility (which must always be checked). As the force reaches zero, the toe leaves the ground and, as there is no impact, there is no impulsive change in momentum and thus the reset map simplifies to the identity map, as previously mentioned.

Phase 3 - Knee Lock $(k l)$. After the swing toe lifts, the biped continues to locomote with flat foot contact between the stance foot and the ground until the swing knee reaches full extension, resulting in an impact which locks the knee. The locking and unlocking of knees could be accomplished by solenoid actuators. Unlike Phases 1 and 2, which are comparatively short, the biped spends a major part of the gait in Phase 3. This ends up being useful as one could say the biped has full actuation in this phase. The constraints imposed on the system in this domain actually reduce the available degrees of freedom in the mechanical configuration to the same as the number of actuators. Thus the robot can be made to move anywhere within the domain of admissibility providing the appropriate constraints aren't violated.

Phase 4 - Heel Strike $(h s)$. The locking of the stance knee which represents the transition to this domain means that both knees are locked in this domain, but the system still has full acutation. This phase ends when the swing heel strikes the ground, resulting in a transition back to the first phase. A coordinate transformation can be constructed to "swap" the angles of the stance leg and swing leg. For the model presented, the new joint angles are given by the following map:

$$
\begin{aligned}
& \mathcal{T}_{q}:\left(q_{8}, q_{7}, q_{6}, q_{5}, q_{4}, q_{3}, q_{2}, q_{1}\right) \\
& \mapsto\left(q_{1}, q_{2}, q_{3}, q_{4}, q_{5}, q_{6}, q_{7}, q_{8}\right) .
\end{aligned}
$$

By choosing the reference frame to be on the torso, the transformation for the base coordinates is simply the identity map. The transformation can then be written as a linear map, $\mathcal{T}=\operatorname{diag}\left(I_{6}, \mathcal{T}_{q}\right)$ which induces pushforward $\mathcal{T}^{*}$. The post-impact state is thus given, as in (47), by $\operatorname{diag}\left(\mathcal{T}, \mathcal{T}^{*}\right) \cdot \Delta_{3 \mathrm{D}}^{4 \rightarrow 1}$ Finally, it should be noted that there are certain choices of control which could result in a bi-periodic orbits due to poor control design.

Table 2

Physical model parameters corresponding to Fig. 16. Massess

\begin{tabular}{|c|c|c|c|c|c|c|c|}
\hline & 1 & $m_{t}$ & $m_{c}$ & $m_{f}$ & $w_{f}$ & $w$ & \\
\hline & 2 & 5 & 1 & .1 & .08 & .10 & \\
\hline$\ell$ & $\ell_{t}$ & $\ell_{c}$ & $r_{a}$ & $r_{f}$ & $r_{h}$ & $r_{t}$ & $r_{T}$ \\
\hline 1 & .175 & .375 & .1 & .139 & .0625 & .25 & .075 \\
\hline
\end{tabular}
and lengths are given in kilograms and meters, respectively.

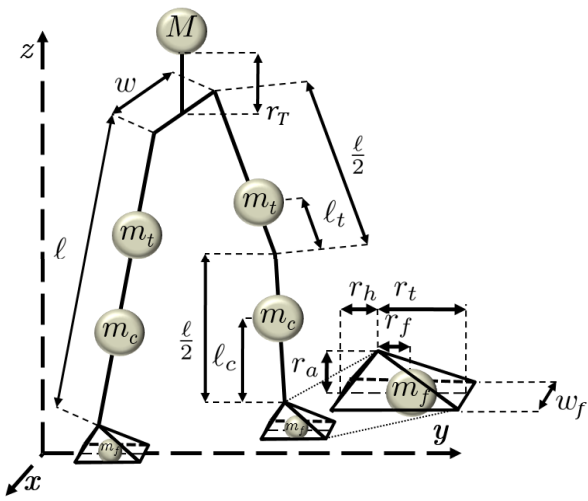

Fig. 16. Configuration of bipedal model.

\subsection{Reduction}

Before constructing the control laws that will be applied to the hybrid control system $\Sigma_{3 \mathrm{D}}$, it is necessary to introduce functional Routhian reduction - the tool that will be used to achieve $3 \mathrm{D}$ walking with a controller designed for an equivalent 2D biped. This form of reduction utilizes almost-cyclic variables, which are analogous to cyclic variables in classical geometric reduction (cf. Marsden and Ratiu [1999]), i.e., these variables are the "symmetries" in the system that will be eliminated through reduction. As with classical reduction, the way in which these variables are eliminated is through a momentum map which describes how momentum is conserved due to the symmetries in the systemthe key difference is that in functional Routhian reduction this momentum map is set to be equal to a function of the almost-cyclic variables rather than a constant. This function can be chosen, which will allow one to pick a specific function that will stabilize the walker to the upright position while simultaneously decoupling the sagittal and coronal dynamics of the system.

Almost-Cyclic Lagrangians. Consider a system with configuration space $\mathcal{Q}=\mathbb{T}^{m} \times S$, where $S$ is called the shape space. Let the coordinates be represented by $q=$ $\left(\varphi^{\prime}, \vartheta^{\prime}\right)^{\prime}$ with $\vartheta \in S$ and almost-cyclic variables $\varphi \in \mathbb{T}^{m}$. A Lagrangian $L_{\lambda}: T \mathbb{T}^{m} \times T S \rightarrow \mathbb{R}$ is almost-cyclic if it takes the form

$$
\begin{aligned}
L_{\lambda}(\varphi, \vartheta, \dot{\varphi}, \dot{\vartheta})=\frac{1}{2}\left(\begin{array}{ll}
\dot{\varphi}^{\prime} & \dot{\vartheta}^{\prime}
\end{array}\right) D_{\lambda}(\vartheta)\left(\begin{array}{c}
\dot{\varphi} \\
\dot{\vartheta}
\end{array}\right) \\
\quad-W_{\lambda}(\varphi, \vartheta, \dot{\vartheta})-V_{\lambda}(\varphi, \vartheta),
\end{aligned}
$$

with

$$
D_{\lambda}(\vartheta)=
$$




$$
\begin{gathered}
\left(\begin{array}{cc}
D_{\varphi}(\vartheta) & D_{\varphi, \vartheta}(\vartheta) \\
D_{\varphi, \vartheta}(\vartheta)^{\prime} & D_{\vartheta}(\vartheta)+D_{\varphi, \vartheta}(\vartheta)^{\prime} D_{\varphi}^{-1}(\vartheta) D_{\varphi, \vartheta}(\vartheta)
\end{array}\right), \\
W_{\lambda}(\varphi, \vartheta, \dot{\vartheta})=\lambda(\varphi)^{\prime} D_{\varphi}^{-1}(\vartheta) D_{\varphi, \vartheta}(\vartheta) \dot{\vartheta} \\
V_{\lambda}(\varphi, \vartheta)=V_{\text {fct }}(\vartheta)-\frac{1}{2} \lambda(\vartheta)^{\prime} D_{\varphi}^{-1}(\vartheta) \lambda(\vartheta),
\end{gathered}
$$

for some function $\lambda: \mathbb{T}^{m} \rightarrow \mathbb{R}^{m}$. Note: $D_{\vartheta}: S \rightarrow$ $\mathbb{R}^{n-m \times n-m}$ and $D_{\varphi}: S \rightarrow \mathbb{R}^{m \times m}$ are positive definite and symmetric.

Momentum Maps. Reduction is based on the concept of a momentum map, $J: T \mathcal{Q} \rightarrow \mathbb{R}^{m}$, which specifies the conserved quantities of a system and is given by

$$
\begin{aligned}
J(\varphi, \vartheta, \dot{\varphi}, \dot{\vartheta}) & =\frac{\partial L_{\lambda}(\varphi, \vartheta, \dot{\varphi}, \dot{\vartheta})}{\partial \dot{\varphi}} \\
& =D_{\varphi, \vartheta}(\vartheta) \dot{\vartheta}+D_{\varphi}(\vartheta) \dot{\varphi}
\end{aligned}
$$

Unlike standard Routhian reduction, in which this map is a constant, functional Routhian reduction allows one to set this map equal to a function $\lambda(\varphi)$.

Functional Routhians. For almost-cyclic Lagrangian $L_{\lambda}$, define the functional Routhian $L_{\mathrm{fct}}: T S \rightarrow \mathbb{R}$ :

$$
L_{\mathrm{fct}}(\vartheta, \dot{\vartheta})=\left[L_{\lambda}(\varphi, \vartheta, \dot{\varphi}, \dot{\vartheta})-\lambda(\varphi)^{\prime} \dot{\varphi}\right]_{J(\varphi, \vartheta, \dot{\varphi}, \dot{\vartheta})=\lambda(\varphi)}
$$

Because $J(\varphi, \vartheta, \dot{\varphi}, \dot{\vartheta})=\lambda(\varphi)$ implies that

$$
\dot{\varphi}=D_{\varphi}^{-1}(\vartheta)\left(\lambda(\varphi)-D_{\varphi, \vartheta}(\vartheta) \dot{\vartheta}\right),
$$

by direct calculation the functional Routhian is given by:

$$
L_{\mathrm{fct}}(\vartheta, \dot{\vartheta})=\frac{1}{2} \dot{\vartheta}^{\prime} D_{\vartheta}(\vartheta) \dot{\vartheta}-V_{\mathrm{fct}}(\vartheta)
$$

Reduction Theorem. Before introducing the reduction theorem, note that for $L_{\lambda}$, the forced EulerLagrange equation can be written as:

$$
\begin{aligned}
& \mathcal{E} \mathcal{L}_{q}\left(L_{\lambda}\right)=D_{\lambda}(\vartheta) \ddot{q}+C_{\lambda}(q, \dot{q}) \dot{q}+ \\
& G_{\lambda}(q)+\mathcal{E} \mathcal{L}_{q}\left(W_{\lambda}(q, \dot{q})\right)+\Upsilon(q, \dot{q}),
\end{aligned}
$$

where $C_{\lambda}(q, \dot{q})$ is obtained from $D_{\lambda}, G_{\lambda}(q)=\frac{\partial V_{\lambda}(q)}{\partial q}$, and $\Upsilon(q, \dot{q})$ represents external forcing. Therefore, the forced Euler-Lagrange equation of $L_{\lambda}$ yields the dynam- ical system

$$
\begin{aligned}
& f_{L_{\lambda}}(q, \dot{q})= \\
& \quad\left(\begin{array}{c}
\dot{q} \\
D_{\lambda}^{-1}(\vartheta)\left(D_{\lambda}(\vartheta) \ddot{q}-\mathcal{E} \mathcal{L}_{q}\left(L_{\lambda}\right)+\Upsilon(q, \dot{q})\right)
\end{array}\right) .
\end{aligned}
$$

In addition, $f_{L_{\mathrm{fct}}}$, the forced vector field corresponding to $L_{\mathrm{fct}}$, is given by

$$
\begin{aligned}
& f_{L_{\mathrm{fct}}}(\vartheta, \dot{\vartheta})= \\
& \quad\left(\begin{array}{c}
\dot{\vartheta} \\
D_{\vartheta}^{-1}(\vartheta)\left(-C_{\mathrm{fct}}(\vartheta, \dot{\vartheta}) \dot{\vartheta}-G_{\mathrm{fct}}(\vartheta)+\Upsilon(q, \dot{q})\right)
\end{array}\right) .
\end{aligned}
$$

obtained from the controlled Euler-Lagrange equation for $L_{\mathrm{fct}}$, given by: $D_{\vartheta}(\vartheta) \ddot{\vartheta}+C_{\mathrm{fct}}(\vartheta, \dot{\vartheta}) \dot{\vartheta}+G_{\mathrm{fct}}(\vartheta)=$ $\Upsilon(q, \dot{q})$. The subsequent theorem assumes that $\Upsilon(q, \dot{q})$ is only dependent on $\vartheta$ and $\dot{\vartheta}$ making equation (88) only a function of $\vartheta$ and $\dot{\vartheta}$ and thus well-defined.

The solutions of these two dynamical systems, $f_{L_{\lambda}}$ and $f_{L_{\mathrm{fct}}}$, are related in the following manner (in a way analogous to the classical Routhian reduction result, see [Marsden and Ratiu, 1999, pp. 260]):

Theorem 19 Let $L_{\lambda}$ be an almost-cyclic Lagrangian with almost-cyclic variable $\varphi \in \mathbb{T}^{m}$ and $L_{\mathrm{fct}}$ the corresponding functional Routhian with shape space $S=\mathbb{R}^{n-m}$. Additionally, let $\Upsilon: T S \rightarrow \mathbb{R}^{n}$ represent external forcing satisfying

(i) $\Upsilon(\vartheta, \dot{\vartheta})$ does not depend on $\varphi, \dot{\varphi}$,

(ii) $\Upsilon_{i}(\vartheta, \dot{\vartheta})=0$, for $i \in\{[1, m] \cap \mathbb{Z}\}$.

(I.e., no external forces act on the almost-cyclic variable.)

Then, $(\varphi(t), \vartheta(t), \dot{\varphi}(t), \dot{\vartheta}(t))$ is a solution to the vector field $f_{L_{\lambda}}$ given by $(87)$ on $\left[t_{0}, t_{F}\right]$ with

$$
\dot{\varphi}\left(t_{0}\right)=D_{\varphi}^{-1}\left(\vartheta\left(t_{0}\right)\right)\left(\lambda\left(\varphi\left(t_{0}\right)\right)-D_{\varphi, \vartheta}\left(\vartheta\left(t_{0}\right)\right) \dot{\vartheta}\left(t_{0}\right)\right),
$$

if and only if $(\vartheta(t), \dot{\vartheta}(t))$ is a solution to the forced vector field $f_{L_{\mathrm{fct}}}$ given by $(88)$ and $(\varphi(t), \dot{\varphi}(t))$ satisfies:

$$
\dot{\varphi}(t)=D_{\varphi}^{-1}(\vartheta(t))\left(\lambda(\varphi(t))-D_{\varphi, \vartheta}(\vartheta(t)) \dot{\vartheta}(t)\right) .
$$

This theorem implies that reduction shapes the sagittal dynamics of the 3D biped to be equivalent to those of the $2 \mathrm{D}$ system. 
Sagittal Restriction. The reduced model is obtained by applying a sagittal restriction, setting all coronal angles to zero, i.e., $q_{1}=\dot{q}_{1}=q_{8}=\dot{q}_{8}=0$, and thereby projecting down onto a submanifold representing the kinematic workspace of the biped in Euclidean coordinates being restricted to $2 \mathrm{D}$. This can be expressed as the hybrid control system

$$
\Sigma_{2 \mathrm{D}}=\left(\Gamma_{2 \mathrm{D}}, \mathcal{X}_{2 \mathrm{D}}, U_{2 \mathrm{D}}, S_{2 \mathrm{D}}, \Delta_{2 \mathrm{D}}, \mathcal{F} \mathcal{G}_{2 \mathrm{D}}\right) .
$$

This control system can be constructed following the same procedure used to construct $\Sigma_{3 \mathrm{D}}$ earlier. Because this model operates exclusively in the sagittal plane, the number of base coordinates necessary to represent the frame at the torso reduces to three. Specifically, with the base coordinates, the generalized coordinates are $q_{2 \mathrm{D}}=\left(p_{s t}^{x}, p_{s t}^{z}, \phi_{s t}^{y}, \vartheta^{\prime}\right)^{\prime}$. This derived model is considered equivalent to the 3D model and indeed equivalence of corresponding dynamics is guaranteed given the the conditions of Theorem 19 are satisfied.

\subsection{Sagittal Control Design}

The gait considered in this paper requires the use of several different control laws. For the sake of obtaining a passivity-based feel, controlled symmetries was taken as the basis for sagittal control design. When combined with a spring-damper (PD) controller to stabilize the torso, controlled symmetries can produce stable walking gaits on point foot models under the assumption of full actuation. This is essentially equivalent to a model with trivial foot behavior, i.e., either flat ground contact or no contact. In order to get nontrivial foot action, additional PD controllers can be added at the ankles and at the non-stance knee. Finally, in order to avoid scuffing, which occurs when the swing toe strikes the ground before desired, a controller is designed to rotate the toe away from the ground with a torque that fades expoentially with the toe's distance from the ground.

Controlled Symmetries. Controlled symmetries, introduced in Spong and Bullo [2005], works by shaping the potential energy a robot to that of a passive biped walking down a slope. A group action effectively "rotates the world" by operating on the potential energy allowing for walking on flat ground given passive walking down a slope. The goal is to combine controlled symmetries with other control laws to achieve stable walking in the 2D sagitally-restricted kneed biped with feet.

To rotate gravity, consider the group action

$$
\begin{aligned}
\Psi_{\gamma}\left(q_{2 \mathrm{D}}\right):\left(p_{s t}^{x}, p_{s t}^{z}, \phi_{s t}^{y}, \vartheta_{1}-\gamma, \vartheta_{2}, \ldots, \vartheta_{6}\right) \\
\longmapsto\left(p_{s t}^{x}, p_{s t}^{z}, \phi_{s t}^{y}, \vartheta_{1} \ldots, \vartheta_{6}\right)
\end{aligned}
$$

for slope angle $\gamma \in \mathbb{S}$ and define the feedback control law

$$
K_{2 \mathrm{D}}^{\gamma}\left(q_{2 \mathrm{D}}\right):=G_{\vartheta}\left(q_{2 \mathrm{D}}\right)-G_{\vartheta}\left(\Psi_{\gamma}\left(q_{2 \mathrm{D}}\right)\right)
$$

with $G_{\vartheta}\left(q_{2 \mathrm{D}}\right)=\frac{\partial V_{2 \mathrm{D}}}{\partial \vartheta}\left(q_{2 \mathrm{D}}\right)$ which in the vector fields

$$
\begin{aligned}
& f_{2 \mathrm{D}, i}^{\gamma}\left(q_{2 \mathrm{D}}, \dot{q}_{2 \mathrm{D}}\right)= \\
& \quad f_{2 \mathrm{D}, i}\left(q_{2 \mathrm{D}}, \dot{q}_{2 \mathrm{D}}\right)+g_{2 \mathrm{D}, i}\left(q_{2 \mathrm{D}}\right) K_{2 \mathrm{D}, i}^{\gamma}\left(q_{2 \mathrm{D}}\right),
\end{aligned}
$$

for $i \in\{1, \ldots, 4\}$.

Spring-Damper Controllers. Motivated by the elasticity the human ankle and by human ankle torque (see $\mathrm{Au}$ et al. [2009]), PD controllers are considered as a means of meeting specific control objectives. For a given joint with angle $\vartheta_{j}$ and angular velocity $\dot{\vartheta}_{j}$, a typical PD controller takes the form

$$
u_{\mathrm{PD}, \vartheta_{j}}\left(q_{2 \mathrm{D}}, \dot{q}_{2 \mathrm{D}}\right)=-k_{\vartheta_{j}}\left(\vartheta_{j}-\vartheta_{\vartheta_{j}, 0}\right)-c_{\vartheta_{j}}\left(\dot{\vartheta}_{j}\right) .
$$

In order to stabilize the torso, (92) requires modification:

$$
u_{\mathrm{PD}, \vartheta_{4}}\left(q_{2 \mathrm{D}}, \dot{q}_{2 \mathrm{D}}\right)=-k_{T}\left(\phi_{s t}^{y}-\vartheta_{T, 0}\right)-c_{T} \omega_{s t}^{y},
$$

where $\phi_{s t}^{y}$ is an Euler angle for the torso and $\omega_{s t}^{y}$ is the body-fixed angular velocity of the torso in the sagittal plane for the model described earlier. The controller is applied at the swing hip, $\vartheta_{4}$. To have the swing foot land in a desirable configuration, and motivated by measurements of human ankle torque during walking, the PD controller (92) is applied at $\vartheta_{6}$. Heuristics has shown that a PD controller at the stance ankle may often contribute to stability and thus (92) is used at $\vartheta_{1}$. In order to get the swing knee moving forward after heel strike, it was necessary to impose $(92)$ on $\vartheta_{5}$.

For simplicity take these controllers to be a set on each domain $i$ such that

$$
U_{\Theta}^{i}= \begin{cases}\{1,4,5,6\}, & i=1, \\ \{1,4,6\}, & i=2,3,4 .\end{cases}
$$

One can observe that the controllers are continuous through a single step with the exception of the controller designed for the swing knee. In a continuous time system, this would mean the torques for smooth control laws would be smooth, but in a hybrid system with impulse-like forces due to impacts, discontinuities will occur in the velocities causing jumps in those control laws which depend on these variables. The keen observer might notice that if equivalent controller parameters were found for $\vartheta_{1}$ and $\vartheta_{6}$, these controllers could be replaced by actual spring-damper mechanisms. These controllers can be combined to construct

$$
K_{2 \mathrm{D}, i}^{\Theta}\left(q_{2 \mathrm{D}}, \dot{q}_{2 \mathrm{D}}\right):=\sum_{j \in U_{\Theta}^{i}} u_{P D, \vartheta_{j}}\left(q_{2 \mathrm{D}}, \dot{q}_{2 \mathrm{D}}\right) \cdot b_{\vartheta, j},
$$


where $b_{\vartheta, j}$ is the $j$-th basis vector for the coordinates $\vartheta$. Applying these controllers to (91) gives

$$
\begin{aligned}
& f_{2 \mathrm{D}, i}^{\gamma, \Theta}\left(q_{2 \mathrm{D}}, \dot{q}_{2 \mathrm{D}}\right)= \\
& f_{2 \mathrm{D}, i}^{\gamma}\left(q_{2 \mathrm{D}}, \dot{q}_{2 \mathrm{D}}\right)+g_{2 \mathrm{D}, i}\left(q_{2 \mathrm{D}}\right) K_{2 \mathrm{D}, i}^{\Theta}\left(q_{2 \mathrm{D}}\right),
\end{aligned}
$$

where gains (Table 3 ) are lumped into superscripts.

Scuffing Prevention Controller. In order to avoid the scuffing phenomenon, a controller can be designed which repels the swing toe from the ground. To minimize interference with the rest of the system's control, the scuffing prevention controller imposes exponential spatial disipation that and thus only makes a significant contribution when the swing toe passes near the floor. This control law thus takes the form

$$
K_{2 \mathrm{D}}^{\beta}\left(q_{2 \mathrm{D}}\right)=-\beta_{1} e^{\beta_{2} \cdot p_{s w t}^{z}\left(q_{2 \mathrm{D}}\right)} \cdot b_{\vartheta, 6},
$$

where $\beta_{1}, \beta_{2} \in \mathbb{R}$ are positive constants and represent the strength of repulsion and spatial dissipation rate, respectively, $b_{\vartheta, 6}$ is the 6 -th basis vector in $\vartheta$, and $p_{s w t}^{z}$ : $S \rightarrow \mathbb{R}$ is the height of the swing toe above the ground. This control law is only desirable when the swing toe is in the air, so appropriate application leads to the following vector fields:

$$
\begin{array}{r}
f_{2 \mathrm{D}, i}^{\gamma, \Theta, \beta}\left(q_{2 \mathrm{D}}, \dot{q}_{2 \mathrm{D}}\right)= \\
f_{2 \mathrm{D}, i}^{\gamma, \Theta}\left(q_{2 \mathrm{D}}, \dot{q}_{2 \mathrm{D}}\right)+ \begin{cases}\mathbf{0}, & \mathrm{i}=1,2, \\
g_{2 \mathrm{D}, i}\left(q_{2 \mathrm{D}}\right) K_{2 \mathrm{D}, i}^{\beta}\left(q_{2 \mathrm{D}}\right), & \mathrm{i}=3,4 .\end{cases}
\end{array}
$$

2D Simulation. Applying the feedback control laws as shown above to the hybrid control system $\Sigma_{2 \mathrm{D}}$ gives the hybrid system

$$
\bar{\Sigma}_{2 \mathrm{D}}^{\gamma, \Theta, \beta}=\left(\Gamma_{2 \mathrm{D}}, \mathcal{X}_{2 \mathrm{D}}, S_{2 \mathrm{D}}, \Delta_{2 \mathrm{D}}, \mathcal{F}_{2 \mathrm{D}}^{\gamma, \Theta, \beta}\right)
$$

where $\mathcal{F}_{2 \mathrm{D}}^{\gamma, \Theta, \beta}=\left\{f_{2 \mathrm{D}, i}^{\gamma, \Theta, \beta}\right\}_{i=1}^{4}$. This hybrid system was simulated with model parameters given in Table 2 and control parameters given in Table 3 . The joint angles and torques resulting from this walking simulation are shown in Fig. 17 and Fig. 18 as dashed lines alongside their counterparts from the later 3D simulation. The

Table 3

Gains for PD controllers, scuffing prevention controller, and controlled symmetries, as well as gravity.

\begin{tabular}{|c|c|c|c|c|c|c|c|}
\hline$k_{\vartheta_{1}}$ & $c_{\vartheta_{1}}$ & $\vartheta_{\vartheta_{1}, 0}$ & $k_{T}$ & $c_{T}$ & $\vartheta_{T, 0}$ & $\beta_{1}$ & $\gamma$ \\
\hline 30 & 0.1 & -0.5 & 100 & 5 & 0 & 10 & 0.05 \\
\hline \hline$k_{\vartheta_{5}}$ & $c_{\vartheta_{5}}$ & $\vartheta_{\vartheta_{5}, 0}$ & $k_{\vartheta_{6}}$ & $c_{\vartheta_{6}}$ & $\vartheta_{\vartheta_{6}, 0}$ & $\beta_{2}$ & $g$ \\
\hline 70 & 1 & 0.5 & 30 & 1 & 0 & 20 & 9.81 \\
\hline
\end{tabular}

stability of the gait can be examined by considering the codimension-one Poincaré section $\mathcal{S}_{4}^{1}$, which is the guard of domain 4 (i.e., heel strike) and involves switching legs. To minimize the perturbations necessary to examine the Poincaré map, one can perturb along a minimal set of bases that span the Poincaré map locally. Holonomic constraints represent restrictions on the degrees of freedom of a system and this shows up in a dropping of rank in the Poincaré map. For this particular model, these minimal bases can be found by moving the fixed frame to the fixed stance foot, then perturbing along angles $\vartheta_{3}, \vartheta_{4}, \vartheta_{6}$ and angular velocities $\vartheta_{1}, \vartheta_{3}, \vartheta_{4}, \vartheta_{6}$. Because the knees are locked, $\vartheta_{2}=\dot{\vartheta}_{2}=\vartheta_{5}=\dot{\vartheta}_{5}$ and one can solve for $\vartheta_{1}$ such that the swing heel is on the ground. Thus the Poincaré map for the orbit drops rank to seven. Through optimization, the fixed point

$$
\begin{aligned}
& \vartheta^{*}=(-.163,0, .245,-.139,0,-.003)^{\prime}, \\
& \dot{\vartheta}^{*}=(-.987,0,1.090,1.068,0, .067)^{\prime},
\end{aligned}
$$

is found. A numerical approximation of a linearization of the Jacobian of the Poincaré map in the seven minimnal bases yields eigenvalues with magnitudes $0.613,0.169$, $0.056, \ldots$. In general, eigenvalues with magnitudes below unity for discrete-time systems indicate stability. The Poincare map for the simulated system is indeed stable thus implying $\left(q^{*}, \dot{q}^{*}\right)$ is a fixed point of a stable periodic orbit which represents the stable walking gait.

One could perform a similar analysis taking a different Poincare section such as the guard for knee lock. This map has two bases more than the guard for heel strike as used above; one may no longer solve for $q_{1}$ and $\dot{\vartheta}_{5}$ becomes arbitrary. By taking this Poincaré section and performing analysis, one would find seven non-zero eigenvalues for the reasons alluded to above.

\subsection{Reduction Control Design}

The scheme depicted in Fig. 15 summarizes the reduction procedure, which consists of first applying an energy shaping control law and then applying a stabilizing controller. The assumptions dictating the validity of functional Routhian reduction require that the system be in flat-foot, single support contact with the ground; for this model, the controller only applies in Phases 3 and 4 . The sagittal controls can be adapted as follows:

$$
\begin{aligned}
K_{3 \mathrm{D}, i}^{\gamma, \Theta}(q, \dot{q}) & :=B_{2 \mathrm{D}}^{3 \mathrm{D}}\left[K_{2 \mathrm{D}, i}^{\gamma}(\pi(q))+\right. \\
& \left.K_{2 \mathrm{D}, i}^{\Theta}(\pi(q))+K_{2 \mathrm{D}, i}^{\beta}\left(\pi(q), \pi^{*}(\dot{q})\right)\right],
\end{aligned}
$$

where $B_{2 \mathrm{D}}^{3 \mathrm{D}}=\left(\frac{\partial q_{2 \mathrm{D}}}{\partial q}\right)^{\prime}$ and $\pi: \mathcal{Q}_{3 \mathrm{D}} \rightarrow S$ is a canonical projection (which induces pushforward $\pi^{*}: T \mathcal{Q}_{3 \mathrm{D}} \rightarrow$ $T S$ ) associated with the sagittal restriction. Applying 

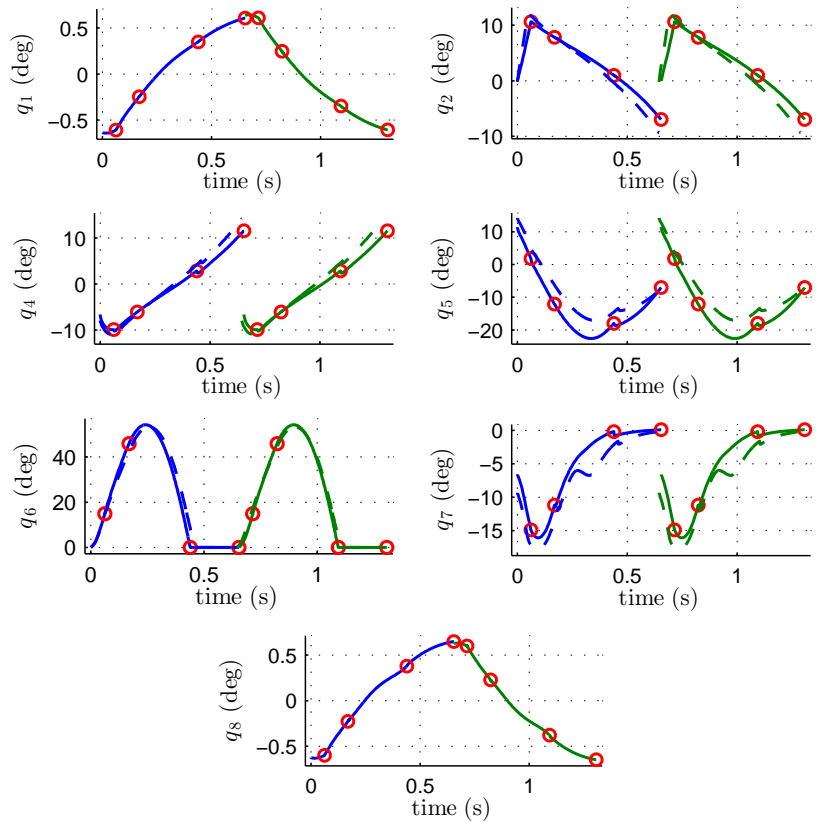

Fig. 17. Joint profiles for the 3D gait (solid) and 2D gait (dashed) over two steps. The small circles represent the points where the discrete transitions occur.
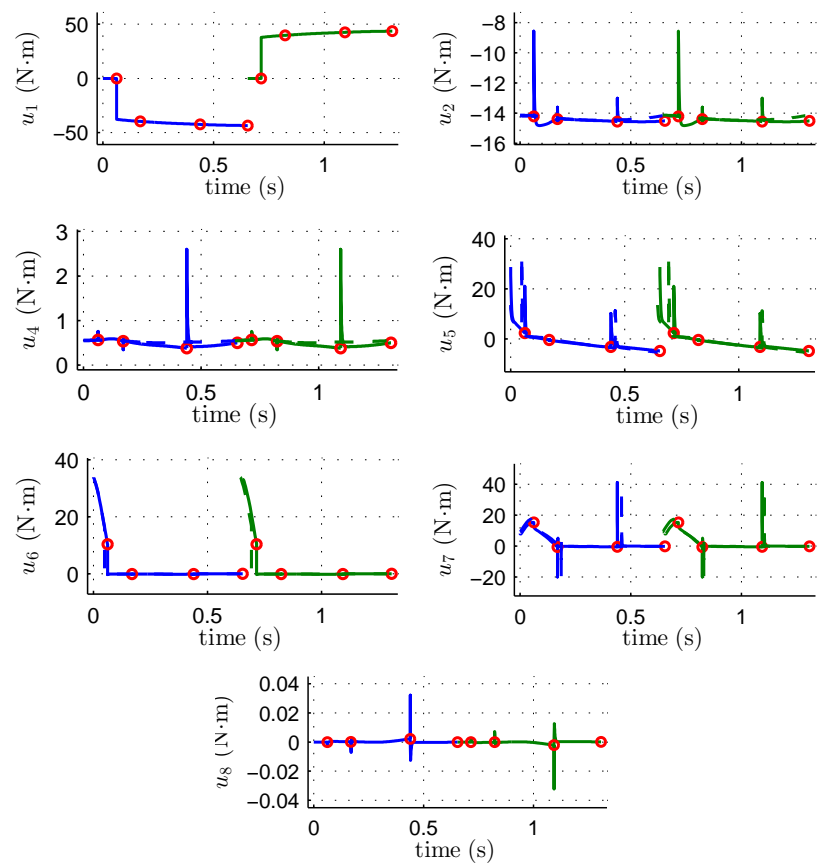

Fig. 18. Torque profiles for the $3 \mathrm{D}$ gait (solid) and $2 \mathrm{D}$ gait (dashed) over two steps. The small circles represent the points where the discrete transitions occur.

this control law yields

$$
f_{3 \mathrm{D}, i}^{\gamma, \Theta, \beta}(q, \dot{q})=f_{3 \mathrm{D}, i}(q, \dot{q})+g_{3 \mathrm{D}, i}(q) K_{3 \mathrm{D}, i}^{\gamma, \Theta, \beta}(q, \dot{q}) .
$$
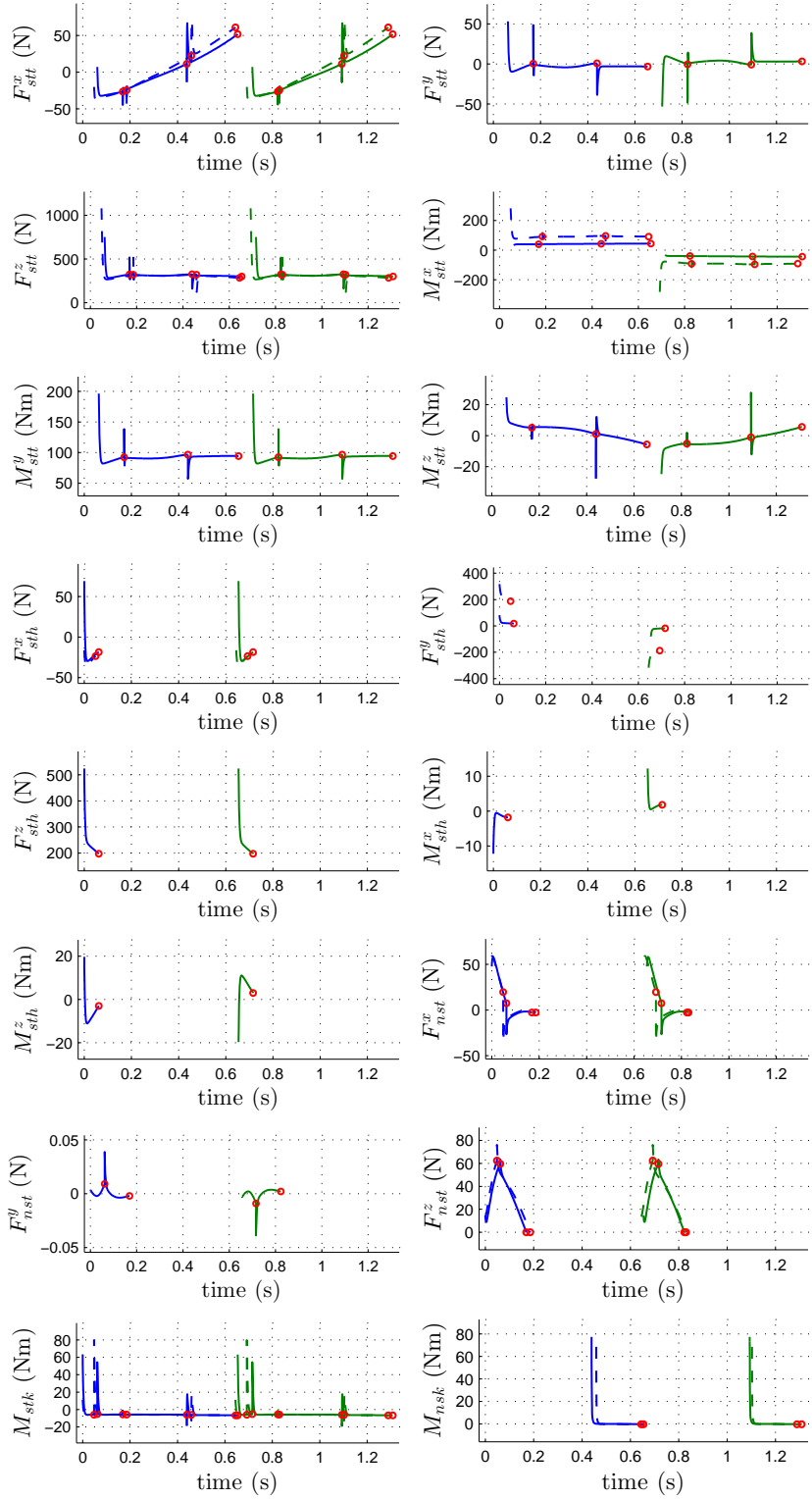

Fig. 19. Ground reaction forces for the 3D gait (solid) and 2D gait (dashed) over two steps. The small circles represent the points where the discrete transitions occur. It is important to verify that these forces keep the system within the domain of admissibility. The spikes above result from the numerous impacts throughout the gait and the reaction of the PD controllers to jumps in velocity.

Lagrangian Shaping Controller. Having an almostcyclic Lagrangian is required for reduction. This controller, therefore, shapes the Lagrangian of the system into the almost-cyclic Lagrangian $L_{\alpha}$ of the form (81), choosing a function $\lambda(\varphi)=-\alpha \varphi$ with $\alpha \in \mathbb{R}$ a positive constant specifying the rate of convergence. Define the control law as

$$
K_{3 \mathrm{D}}^{\gamma, \Theta, \beta, \alpha}(q, \dot{q}):=B_{3 \mathrm{D}}^{-1}\left(H_{3 \mathrm{D}}(q, \dot{q})+M_{3 \mathrm{D}}(q) D_{\alpha}^{-1}(\vartheta)\right.
$$




$$
\left.\cdot\left[D_{\alpha}(\vartheta) \ddot{q}-\mathcal{E}_{\mathcal{L}_{q}}\left(L_{\alpha}(q, \dot{q})\right)+K_{3 \mathrm{D}}^{\gamma, \Theta, \beta}(q, \dot{q})\right]\right)
$$

with $D_{\alpha}(\vartheta)$ the shaped inertia matrix as given in (82) and $\mathcal{E} \mathcal{L}_{q}\left(L_{\alpha}(q, \dot{q})\right)$ given in (86). Applying this control law on the first two domains yields the vector field

$$
\begin{aligned}
f_{3 \mathrm{D}, i}^{\gamma, \Theta, \beta, \alpha}(q, \dot{q}) & = \\
f_{3 \mathrm{D}}(q, \dot{q}) & + \begin{cases}\mathbf{0}, & i=1,2, \\
g_{3 \mathrm{D}}(q) K_{3 \mathrm{D}, i}^{\gamma, \Theta, \beta}(q, \alpha), & i=3,4 .\end{cases}
\end{aligned}
$$

Reduction Surface Stabilization. In order to enjoy the decoupling effects of functional Routhian reduction, the system must operate on the surface where reduction is valid. Doing so will result in the system satisfying (90). This motivates the output function

$$
y_{z}(q, \dot{q})=\dot{\varphi}+D_{\varphi}^{-1}(\vartheta)\left(\alpha \varphi+D_{\varphi, \vartheta}(\vartheta) \dot{\vartheta}\right),
$$

which has relative degree one. Driving this output to zero will drive the system to the forward-invariant surface

$$
\mathcal{R}=\left\{\left(\begin{array}{c}
q \\
\dot{q}
\end{array}\right) \in T \mathcal{Q}_{3 \mathrm{D}}: y_{z}(q, \dot{q})=0\right\} .
$$

Using feedback linearization (cf. Sastry [1999]), a control law which results in $y_{z} \rightarrow 0$ is

$$
K_{3 \mathrm{D}}^{\varepsilon}(q, \dot{q}):=\left[\left(L_{g_{3 \mathrm{D}}} y_{z}\right)^{-1}\left(-L_{f_{3 \mathrm{D}}^{\gamma, \Theta, \beta, \alpha}} y_{z}-\varepsilon y_{z}\right)\right] \cdot b_{\varphi}
$$

where $b_{\varphi}$ is a basis vector corresponding to the almostcyclic coordinate $\phi$ and $\varepsilon \in \mathbb{R}$ is a positive constant specifying rate of convergence. Applying this control law gives the dynamical systems

$$
\begin{array}{r}
f_{3 \mathrm{D}, i}^{\gamma, \Theta, \beta, \alpha, \varepsilon}(q, \dot{q})= \\
f_{3 \mathrm{D}, i}^{\gamma, \Theta, \beta, \alpha}(q, \dot{q})+ \begin{cases}\mathbf{0}, & i=1,2, \\
g_{3 \mathrm{D}, i}(q) K_{3 \mathrm{D}}^{\varepsilon}(q, \dot{q}), & i=3,4 .\end{cases}
\end{array}
$$

3D Simulation. Applying the preceding control laws to the hybrid control system $\Sigma_{3 \mathrm{D}}$ gives the hybrid system

$$
\bar{\Sigma}_{3 \mathrm{D}}^{\gamma, \Theta, \beta, \alpha, \varepsilon}=\left(\Gamma_{3 \mathrm{D}}, \mathcal{X}_{3 \mathrm{D}}, \mathcal{S}_{3 \mathrm{D}}, \Delta_{3 \mathrm{D}}, \mathcal{F}_{3 \mathrm{D}}^{\gamma, \Theta, \beta, \alpha, \varepsilon}\right),
$$

where $\mathcal{F}_{3 \mathrm{D}}^{\gamma, \Theta, \beta, \alpha, \varepsilon}=\left\{f_{3 \mathrm{D}, i}^{\gamma, \Theta, \beta, \varepsilon, \varepsilon}\right\}_{i=1}^{4}$. This hybrid system contains the same control laws implemented on the $2 \mathrm{D}$ model in addition to the reduction control laws and the additional control gains $\alpha=10$ and $\varepsilon=25$ are used.
The joint angles and torques for this walking simulation are shown in Fig. 17 and Fig. 18 as solid lines and the $2 \mathrm{D}$ simulation is shown in dashed lines for comparison. The closeness of trajectories can be readily observed. The discrepancy in the nonstance ankle pitch $q_{7}$ can be attributed to the scuffing prevention controller: in the $3 \mathrm{D}$ model, the biped sways so the toe stays farther away from the ground, thus reducing the need for the scuffing prevention controller. $q_{1}$ and $q_{8}$ are identical as $q_{8}$ is commanded to allow for flat edge contact upon heel strike.

Using a similar procedure to that conducted in the $2 \mathrm{D}$ simulation, a fixed point is found,

$$
\begin{aligned}
& q^{*}=(-.011,-.115,0, .199,-.115,0,-.001,-.011)^{\prime}, \\
& \dot{q}^{*}=(-.015,-.844,0, .931,1.574,0, .010,-.007)^{\prime},
\end{aligned}
$$

Due to the numerical complexity of the model, the authors were unable to approximate the eigenvalues of the system; this is indeed a troublesome area for many researchers due to the limitations of numerical methods. Yet the system appears stable as the trajectory does not diverge from the limitcycle after many steps.

The joint angles and torques for the $2 \mathrm{D}$ and $3 \mathrm{D}$ section are shown overlaid in Figs. 17 and 18 while tiles are shown in Fig. 20. The main observation from comparison is that the systems behave very similarly. However, differences in the models arise due to the inability of reduction to cope with external forcing (i.e., double support) and the lack of invariance of the reduction surface $\mathcal{R}$ to impacts which differ between models. Moreover, springdamper systems like what is created through the application of PD control is known be difficult to obtain accuracy through numerical integration. But after each impact, the reduction surface stabilization controller (98) acts to return the system to the surface $\mathcal{R}(97)$ so the system still remains close to the surface and this surface is exponentially stable. Thus the sagittal and coronal dynamics are decoupled through the use of reduction indicating the effectiveness of functional Routhian reduction for designing gaits for 3D bipeds. Moreover, a reduction in model complexity is demonstrated which simplifies controller design. Finally, the reaction forces are shown for both simulations in Fig. 19 and were used to verify the domain of admissibility. For more detail along this line of research, see Sinnet [2011].

\section{Summary and Open Problems}

\subsection{Summary}

This paper has attempted to provide the reader with an introduction to the area of $3 \mathrm{D}$ bipedal locomotion. Section 2 emphasized that, in locomotion, steady-state behaviors correspond to periodic solutions and not static 


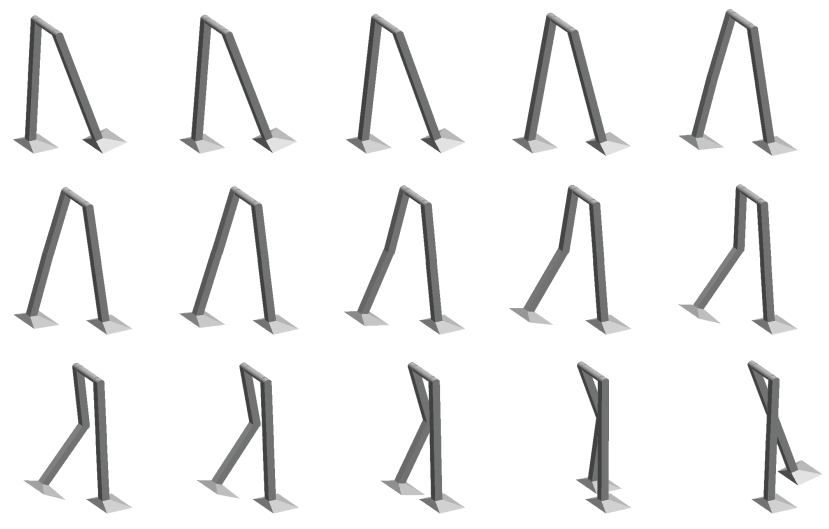

Fig. 20. Sample gait for 3D simulation

equilibria. Consequently, Poincaré first return maps are key in characterizing stability of periodic solutions. While some of the technical details associated with Poincaré maps are more delicate for hybrid models, at a fundamental level, the method works essentially the same as for non-hybrid systems described by a single set of ordinary differential equations. The study of periodic solutions of hybrid models was placed before the modeling section for two reasons. First of all, stability is dear to the heart of a control theorist, so its study in hybrid systems would be of interest independent of the underlying physical phenomena. Secondly, it hopefully placed the reader in the frame of mind of seeking to understand in Sect. 3 just how a mixture of continuous and discrete behaviors could arise from Lagrangian dynamics.

Section 3 presented models. The models vary depending on both the characteristics of the robot as well as the characteristics of the gait being studied, and this coupling occurs because of the contact forces and moments, called the contact wrench, between the robot and the ground. Because much of the control-oriented literature on locomotion has been ambiguous on the calculation of the contact wrench, the interpretation of its components, and its primal role in determining the validity of a given model, the modeling section paid extra attention to this aspect of bipedal locomotion. The researcher wishing more detail on computing the unconstrained dynamic model of a bipedal robot, that is, the robot without any interactions with its environment other than gravity, can consult numerous texts.

Section 4 gave a broad overview of how bipedal walking gaits are being designed and controlled. Emphasis was given to how the various control approaches relate to the underlying model. The vast majority of the literature uses one or more of the simplified models illustrated in Fig. 5. Sections 5 through 7 provided more depth on two approaches to control design for achieving asymptotically stable, periodic walking gaits in 3D bipedal robots. Both of these approaches use the full hybrid dynamic model of the robot and gait. The first approach focused on gaits exhibiting underactuation, while the second fo- cused on gaits composed of a series of phases.

\subsection{Challenges}

The paper will conclude with a non-exhaustive list of questions that one or more of the authors find challenging and important.

Aperiodic Gaits: The models, analysis procedures and control designs presented in the paper have focused on periodic locomotion. It is important to move beyond this assumption. Some preliminary results can be found in Yang et al. [2007, 2009]. A notion of stability based on expected time to a fall is developed in Byl and Tedrake [2008a], Byl and Tedrake [2008b] and Byl and Tedrake [2009] for low-dimensional models. Qualitative indicators of stability are discussed in Su and Dingwell [2007] and references therein, especially in relation to falls and injuries in the elderly. There is room for improved notions of stability of aperiodic walking gaits. Two cases where aperiodic gaits arise naturally are walking on uneven ground and maneuvering a biped around obstacles. These are discussed next.

Walking on Uneven Ground: This tutorial emphasized walking on flat ground. Much less is known about the problem of bipedal walking on unknown, uneven ground. The mechanical design of feet for assuring good ground contact has been studied in Yamaguchi et al. [1995]. Heuristics for maintaining stability on slight inclines were studied in Kim et al. [2007], and for more aggressive ground variations in Hodgins and Raibert [1991], Kajita and Tani [1997], Shih and Chiou [1998], Huang et al. [2000], Shimizu et al. [2007], Erez and Smart [2007], Yin et al. [2007]. A virtual constraint based human-inspired control methodology was experimentally realized in Kolathaya and Ames [2012]. Analytical work is just beginning Manchester et al. [2011a], Dai and Tedrake [2013], Park et al. [2012, 2013]. The role of compliance in locomotion on uneven terrain has been emphasized in Hodgins and Raibert [1991], Saranli et al. [2001], Daley et al. [2006], Daley and Biewener [2006], Hashimoto et al. [2006], Ogino et al. [2007].

Maneuvering: Relatively few papers have addressed the issue of maneuvering for bipedal robots, and even fewer have attempted to provide stability guarantees. Heuristic methods have included turning motions based on the duty ratios of the two legs; allowing the feet to slip when rotating with respect to the ground; other trialand-error methods Shih [1999], Sakagami et al. [2002], Miura et al. [2008], Yagi and Lumelsky [2000]. The references Gregg and Spong [2008, 2009] have developed an elegant and rigorous setting for stable walking and steering of fully actuated 3D robots using a variant of functional Routhian reduction (the basic ideas behind this form of geometric reduction were discussed in Sect. 7). Steering is achieved by adjusting the yaw set point of 
the within-stride passivity-based controller. How to turn without violating unilateral ground contact constraints is not explained in this work. In Powell et al. [2013], Dantam et al. [2013], a method for speed regulation in 3D walking robots is presented through the notion of motion primitives and transitions based upon hybrid zero dynamics surfaces. This gives formal guarantees of correct behavior when walking at different speeds and transitioning between them, along with methods for automatically synthesizing code that experimentally realizes the formal results.

Impact Models: Work in Miossec and Aoustin [2002] indicates that the approach currently used to represent an inelastic impact of the swing leg with the ground essentially rules out a nontrivial double support phase. In human walking, the double support phase accounts for approximately $20 \%$ of the gait. A commonly held opinion in the locomotion field is that compliance is essential for achieving such gaits. It would be very useful to find impact models, even very approximate ones, that will allow such gaits in robots with "stiff" feet and limbs. A step in this direction has been taken by $\mathrm{Mu}$ and $\mathrm{Wu}$ [2006].

Standing and Push Recovery with Passive Feet: Standing is challenging for robots with passive ankles. Regaining a quiescent standing position after being pushed is even harder. The existing literature is rich for robots with actuated ankles; see Pratt et al. [2012], Koolen et al. [2012] and references therein.

Computation of Periodic Solutions: The approach in Sect. 5 combines the determination of virtual constraints and open-loop, periodic solutions to the hybrid model of a bipedal gait. Our experience is that finding periodic solutions in this manner is difficult due to numerous local minima. The problem is even more difficult when seeking solutions that are energy efficient, in addition to being periodic. Other approaches to finding periodic orbits, such as those in Srinivasan and Ruina [2006] and Posa et al. [2014], need to be considered when designing feedback laws through virtual constraints.

Computing the Domain of Attraction: Computing the domain of attraction of a stable equilibrium point has been well-studied in the area of dynamical systems. What remains an almost completely unexplored area is computing the domain of attraction for periodic solutions, even for dynamical systems. Important initial work in hybrid systems is available in Tedrake et al. [2010], Manchester et al. [2011b]. Characterizing the domain of attraction of a periodic walking gait for a bipedal robot model is an important consideration with respect to the eventual implementation of the corresponding feedback controller on the actual biped. There will naturally be errors in the model of the biped, and accounting for these while estimating the domain of attraction would be very useful when passing from simulation to experimentation.

Determining the "Correct" Hybrid Model of a Human-Like Biped: This paper considered two distinct hybrid models. In the literature on bipedal robots, hybrid models have been considered with everywhere from one discrete domain or phase (which is the case for most models considered), to five. This raises the natural question: given an anthropomorphic bipedal robot, what is the hybrid model that best captures the behavior of this system? In particular, what is the model so that when stable walking gaits are found, these gaits are as human-like as possible? Answering this question would be important because, if a single "most human-like" hybrid model could be found, this could be used as the canonical model for studying controller development for obtaining anthropomorphic walking for bipedal robots. Yet, in order to answer this question in a formal manner, it seems necessary to develop a metric that can measure the human-like nature of bipedal walking.

Metrics for Human-Like Walking: There are a wide variety of controllers that can yield walking in a wide array of bipedal robot models; for example, two types of controllers were considered in this paper that yielded walking for two different bipeds. A question is: which controller for which hybrid model yields the most human-like walking Miura et al. [2011]? In order to answer this question, a metric on the distance between walking gaits is needed, i.e., a metric for comparing the distance between solutions of hybrid systems even in the case when the hybrid models are different. This could then be used to both compare different controllers - through a comparison of the resulting periodic solutions - and to compare robotic walking with human walking-by computing the distance between periodic solutions for bipedal robots and periodic solutions corresponding to real human walking data (obtained through motion capture, the use of sensors, or a combination of both).

Develop New Cost-Functions: Once a viable metric for comparing walking gaits has been found, it can be used to develop new cost-functions. Cost functions play a fundamental role in developing controllers for bipedal robots since parameters for the controllers are typically found by minimizing a specific cost function, e.g., in the case of hybrid zero dynamics, the parameters in the virtual constraints that are not fixed by the conditions that enforce hybrid invariance are determined by minimizing a cost function. Yet, currently, there are only two cost functions that are typically considered: the integral-squared torque per step length (as was considered in this paper, see (70)) and the specific cost of transport. There is, of course, no guarantee that minimizing these cost functions yields human-like walking. Therefore, through notion of the distance of a specific gait from being human-like, it may be possible to de- 
velop better cost functions, i.e., cost functions such that, when minimized, yield periodic solutions that are very "close" to human-like walking gaits. This is potentially a very important problem because it might allow for the development of controllers for bipedal robots that yield more human-like walking gaits and are thus naturally transferable to controllers for prosthetic devices.

Foot Shape and Prosthetics: Passive robots as studied in Collins et al. [2001], which can stably walk down small slopes under the power of gravity, are inspiring the design of semi-passive robots, which can walk on flat ground with very low energy consumption; see Collins et al. [2005]. Spherically shaped feet have proven especially useful in the design of such robots. With spherical feet, the model of the contact between the ground and the foot is different from the cases studied in this paper, though it involves underactuation similar to the pointfoot contact model. In the case of $2 \mathrm{D}$ walking, the control law proposed in Sect. 5 has been extended to spherical feet in Kinugasa et al. [2009]. The 3D case is open and interesting. Indeed, in human locomotion, as noted in Hansen et al. [2004] and Adamczyk et al. [2006], the stance ankle and foot together approximate the rolling motion of a wheel, imparting energy efficiency to the human gait. This is one of the reasons that foot prosthetics have a spherical shape. In a related line of investigation, Srinivasan et al. [2008] and Srinivasan et al. [2009] have tied locomotion models based on virtual constraints to human walking data, for both normal gaits and gaits of transtibial prosthesis users. It is argued that the models can be a useful analytical tool for making more informed design and selection of prosthetic components for arriving at more energy efficiency gaits in prosthesis users. It would be interesting to extend this work to address gait stability in the presence of passive or active prostheses. The application to lower-limb prostheses of control laws conceived for bipeds is presented in Gregg and Sensinger [2013].

Other: Many other interesting questions arise, ranging from reflex actions to enhance stability under large perturbations, to bipedal robo;t safety when operating around humans, manipulation of objects, navigation, etc.

\section{References}

P. G. Adamczyk, S. H. Collins, and A. D Kuo. The advantages of a rolling foot in human walking. J. Exp. Biol., 209:3953-3963, 2006.

M. Ahmadi and M. Buehler. Control passive dynamic running experiment with the ARL Monopod II. IEEE Trans. Robot., 22(5):974-986, October 2006.

R. McN. Alexander. Three uses for springs in legged locomotion. Int. J. Robot. Res., 9(2):53-61, 1990.

A. D. Ames. A Categorical Theory of Hybrid Systems. PhD thesis, University of California, Berkeley, 2006.
A. D. Ames. First steps toward automatically generating bipedal robotic walking from human data. In K. R. Kozlowski, editor, Robotic Motion and Control 2011, Gronów, 15-17 June 2011, volume 422 of LNCIS, pages 89-116. Springer, 2011.

A. D. Ames. First steps toward underactuated humaninspired bipedal robotic walking. In Proc. 2012 IEEE Int. Conf. Robot. Autom. (ICRA), pages 1011-1017, St. Paul, May 2012.

A. D. Ames. Human-inspired control of bipedal walking robots. To appear in IEEE Trans. Autom. Contr., 2014.

A. D. Ames and M. J. Powell. Towards the unification of locomotion and manipulation through control Lyapunov functions and quadratic programs. In Control of Cyber-Physical Systems, pages 219-240. Springer, 2013.

A. D. Ames, R. D. Gregg, and M. W. Spong. A geometric approach to three-dimensional hipped bipedal robotic walking. In Proc. 46th IEEE Conf. Decis. Contr. (CDC), pages 5123-5130, New Orleans, 2007.

A. D. Ames, R. W. Sinnet, and E. D. B. Wendel. Threedimensional kneed bipedal walking: A hybrid geometric approach. In R. Majumdar and P. Tabuada, editors, Proc. 12th Int. Conf. Hybrid Syst.: Comp. Contr.(HSCC), San Francisco, CA, 13-15 April 2009, volume 5469 of $L N C S$, pages 16-30, Berlin, 2009. Springer-Verlag.

A. D. Ames, R. Vasudevan, and R. Bajcsy. Human-data based cost of bipedal robotic walking. In Proc. 14th Int. Conf. Hybrid Syst.: Comp. Contr. (HSCC), pages 153-162, Chicago, 2011.

A. D. Ames, E. A. Cousineau, and M. J. Powell. Dynamically stable robotic walking with nao via humaninspired hybrid zero dynamics. In Proc. 15th Int. Conf. Hybrid Syst.: Comp. Contr. (HSCC), Beijing, April 2012a.

A. D. Ames, K. Galloway, and J. W. Grizzle. Control Lyapunov functions and hybrid zero dynamics. In Proc. 51st IEEE Conf. Decis. Contr., pages $6837-$ 6842, Maui, 2012b.

A. D. Ames, K. Galloway, J. W. Grizzle, and K. Sreenath. Rapidly exponentially stabilizing control Lyapunov functions and hybrid zero dynamics. Submitted to IEEE Trans. Autom. Contr., 2012c.

S. O. Anderson, M. Wisse, C. G. Atkeson, J. K. Hodginsand G. J. Zeglin, and B. Moyer. Powered bipeds based on passive dynamic principles. In 5th IEEE/RAS Int. Conf. Humanoid Robots, pages 110-116, Tsukuba, December 2005.

S. K. Au, J. Weber, and H. Herr. Powered anklefoot prosthesis improves walking metabolic economy. IEEE Trans. Robot., 25(1):51-66, 2009.

P. Bézier. Numerical Control: Mathematics and Applications. John Wiley \& Sons, New York, 1972.

R. Blickhan. The spring mass model for running and hopping. J. Biomech., 22(11-12):1217-1227, 1989.

E. Borzova and Y. Hurmuzlu. Passively walking five-link robot. Automatica, 40(4):621-629, 2004. 
B. Brogliato. Nonsmooth Mechanics: Models, Dynamics and Control. Springer-Verlag, New York, 1999.

K. Byl and R. Tedrake. Metastable walking on stochastically rough terrain. In Robot.: Sci. Syst., pages 230237, Zürich, June 2008a.

K. Byl and R. Tedrake. Approximate optimal control of the compass gait on rough terrain. In Proc. 2008 IEEE Int. Conf. Robot. Autom. (ICRA), Pasadena, May 2008b.

K. Byl and R. Tedrake. Metastable walking machines. Int. J. Robot. Res., 28(8):1040-1064, 2009.

C. Chevallereau, G. Abba, Y. Aoustin, F. Plestan, E. R. Westervelt, C. Canudas-de-Wit, and J. W. Grizzle. RABBIT: A testbed for advanced control theory. IEEE Contr. Syst. Mag., 23(5):57-79, October 2003.

C. Chevallereau, E. R. Westervelt, and J. W. Grizzle. Asymptotically stable running for a five-link, fouractuator, planar bipedal robot. Int. J. Robot. Res., 24 (6):431 - 464, June 2005.

C. Chevallereau, D. Djoudi, and J. W. Grizzle. Stable bipedal walking with foot rotation through direct regulation of the zero moment point. IEEE Trans. Robot., 25(2):390-401, 2008.

C. Chevallereau, G. Bessonnet, G. Abba, and Y. Aoustin. Bipedal Robots: Modeling, Design and Walking Synthesis. Wiley/ISTE, New York, 2009a.

C. Chevallereau, J. W. Grizzle, and C.-L. Shih. Asymptotically stable walking of a five-link underactuated 3D bipedal robot. IEEE Trans. Robot., 25(1):37-50, 2009b.

J. H. Choi and J. W. Grizzle. Planar bipedal walking with foot rotation. In Proc. Amer. Contr. Conf. (ACC), pages 4909-4916, Portland, 2005.

S. Collins, A. Ruina, R. Tedrake, and M. Wisse. Efficient bipedal robots based on passive-dynamic walkers. Science, 307:1082-1085, February 2005.

S. H. Collins, M. Wisse, and A. Ruina. A 3-D passive dynamic walking robot with two legs and knees. Int. J. Robot. Res., 20:607-615, 2001.

H. Dai and R. Tedrake. $L_{2}$-gain optimization for robust bipedal walking on unknown terrain. In Proc. 2013 IEEE Int. Conf. Robot. Autom. (ICRA), pages 31163123, Karlsruhe, May 2013.

M. A. Daley and A. A. Biewener. Running over rough terrain reveals limb control for intrinsic stability. Proc. Nat. Acad. Sci. USA, 103(42):15681-15686, October 2006.

M. A. Daley, J. R. Usherwood, G. Felix, and A. A. Biewener. Running over rough terrain: guinea fowl maintain dynamic stability despite a large unexpected change in substrate height. J. Exp. Biol., 209:171-187, 2006.

N. Dantam, A. Hereid, A. D. Ames, and M. Stilman. Correct software synthesis for stable speed-controlled walking. In Robot.: Sci. Syst., Berlin, June 2013.

M. Dickinson, C. Farley, R. Full, M. Koehel, R. Kram, and S. Lehman. How animals move: An integrative view. Science, 288:100-106, April 2000.

E. Dombre and W. Khalil. Modeling, Identification and
Control of Robots. Hermes Sciences, Europe. Taylor \& Francis Books, Inc., New York, 2002.

T. Erez and W. D. Smart. Bipedal walking on rough terrain using manifold control. In Proc. 2007 IEEE/RSJ Int. Conf. Intell. Robots Syst. (IROS), pages 15391544, San Diego, October 2007.

B. Espiau and A. Goswami. Compass gait revisited. In Proc. IFAC Symp. Robot Contr., pages 839-846, Capri, September 1994.

C. T. Farley, J Glasheen, and T. A. McMahon. Running springs: Speed and animal size. J. Exp. Biol., pages 71-86, 1993.

A. F. Filippov. Differential equations with discontinuous right hand side. AMS Transl., 62:199-231, 1960.

R. A. Freeman and P. V. Kokotović. Robust Nonlinear Control Design. Birkhäuser, 1996.

R. Full and D.E. Koditschek. Templates and anchors: Neuromechanical hypotheses of legged locomotion on land. J. Exp. Biol., 202:3325-3332, December 1999.

R. J. Full and C. T. Farley. Musculoskeletal dynamics in rhythmic systems - a comparative approach to legged locomotion. In J. M. Winters and P. E. Crago, editors, Biomech. Neural Contr. Posture Movement. SpringerVerlag, New York, 2000.

K. Galloway, K. Sreenath, A. D. Ames, and J. W. Grizzle. Torque saturation in bipedal robotic walking through control Lyapunov function based quadratic programs. arXiv:1302.7314 [cs.SY], 2013.

M. Garcia, A. Chatterjee, A. Ruina, and M. Coleman. The simplest walking model: Stability, complexity, and scaling. J. Biomed. Eng., 120(2):281-288, April 1998.

J.-B. Geursen, D. Altena, and C.-H. Massen. A model of the standing man for the description of his dynamic behaviour. Agressologie, 17(63-69), 1976.

C. Glocker and F. Pfeiffer. Dynamical systems with unilateral contacts. Nonlinear Dynamics, 3:245-259, 1992.

R. Goebel, R. Sanfelice, and A. Teel. Hybrid dynamical systems. IEEE Contr. Syst. Mag., 29(2):28-93, April 2009.

H. Goldstein, C. Poole, and J. Safko. Classical Mechanics. Addison-Wesley, San Francisco, 3 edition, 2002.

J. M. Goncalves, A. Megretski, and M. A. Dahleh. Global stability of relay feedback systems. IEEE Trans. Autom. Contr., 46(4):550-562, April 2001.

D. M. Gorinevsky, A. M. Formal'sky, and A. Schneider. Force control of robotic systems. CRC Press, Boca Raton, 1997.

A. Goswami. Postural stability of biped robots and the foot-rotation indicator (FRI) point. Int. J. Robot. Res., 18(6):523-533, June 1999.

R. D. Gregg and J. W. Sensinger. Towards biomimetic virtual constraint control of a powered prosthetic leg. IEEE Transactions on Control Systems Technology, 22(1):246-254, 2013.

R. D. Gregg and M. W. Spong. Reduction-based control with application to three-dimensional bipedal walking robots. In Proc. Amer. Contr. Conf. (ACC), pages 
880-887, Seatle, June 2008.

R. D. Gregg and M. W. Spong. Reduction-based control of three-dimensional bipedal walking robots. Int. J. Robot. Res., 2009.

J. A. Grimes and J. W. Hurst. The design of ATRIAS 1.0 a unique monopod, hopping robot. In Proc. 15th Int. Conf. Climbing Walking Robots (CLAWAR), pages 548-554, Baltimore, July 2012.

J. W. Grizzle. Pub lications on robotics and control. www.eecs.umich.edu/grizzle/papers/ robotics.html, March 2014a.

J. W. Grizzle. Dynamic leg locomotion. www. youtube.com/user/DynamicLegLocomotion, March 2014b.

J. W. Grizzle, G. Abba, and F. Plestan. Asymptotically stable walking for biped robots: Analysis via systems with impulse effects. IEEE Trans. Autom. Contr., 46: 51-64, January 2001.

J. W. Grizzle, J. H. Choi, H. Hammouri, and B. Morris. On observer-based feedback stabilization of periodic orbits in bipedal locomotion. In Proc. 13th Int. Conf. Methods Models Autom. Robot. (MMAR), Szczecin, August 2007.

F. Grognard and C. Canudas de Wit. Design of orbitally stable zero dynamics for a class of nonlinear systems. Syst. Contr. Let., 51:89-103, 2004.

J. Guckenheimer and P. Holmes. Nonlinear Oscillations, Dynamical Systems, and Bifurcations of Vector Fields, volume 42 of Applied Mathematical Sciences. Springer-Verlag, New York, corrected second printing edition, 1996.

J. Guckenheimer and S. Johnson. Planar hybrid systems. In Hybrid Systems II, Lecture Notes in Computer Science, pages 203-225. Springer-Verlag, 1995.

W. M. Haddad, V. S. Chellaboina, and S. G. Nersesov. Impulsive and Hybrid Dynamical Systems: Stability, Dissipativity, and Control. Princeton University Press, Princeton, NJ, 2006.

A. H. Hansen, D. S. Childress, and E. H. Knox. Roll-over shapes of human locomotor systems: Effect of walking speed. Clin. Biomech., 19(4):407-414, 2004.

K. Hashimoto, Y. Sugahara, H. Sunazuka, C. Tanaka, A. Ohta, M. Kawase, H. O. Lim, and A. Takanishi. Biped landing pattern modification method with nonlinear compliance control. In Proc. 2006 IEEE Int. Conf. Robot. Autom. (ICRA), pages 1213-1218, Orlando, May 2006.

J. Hauser and C. C. Chung. Converse Lyapunov functions for exponentially stable periodic orbits. Syst. Contr. Let., 23:27-34, 1994.

Andrei Herdt, Holger Diedam, Pierre-Brice Wieber, Dimitar Dimitrov, Katja Mombaur, and Moritz Diehl. Online walking motion generation with automatic footstep placement. Advanced Robotics, 24(5-6):719737, 2010.

K. Hirai, M. Hirose, Y. Haikawa, and T. Takenaka. The development of Honda humanod robot. In Proc. 1998 IEEE Int. Conf. Robot. Autom. (ICRA), volume 2, pages 1321-1326, Leuven, May 1998.
I. A. Hiskens. Stability of hybrid limit cycles: application to the compass gait biped robot. In Proc. 40th IEEE Conf. Decis. Contr., pages 774-779, Orlando, December 2001.

J. K. Hodgins and M. H. Raibert. Adjusting step length for rough terrain locomotion. IEEE Trans. Robot. Autom., 7(3):289-298, 1991.

A. L. Hof, M. G. J. Gazendam, and W. E. Sinke. The condition for dynamic stability. J. Biomech., 38(1): 1-8, 2005.

J.M. Hollerbach, I.W. Hunter, and J. Ballantyne. A comparative analysis of actuator technologies for robotics. MIT Press. Khatib, O. and Canny, J., and LozanoPerez, T., Cambridge, 1992.

P. Holmes, R. J. Full, D. E. Koditschek, and J. Guckenheimer. The dynamics of legged locomotion: Models, analyses, and challenges. SIAM Rev., 48(2):207-304, 2006.

Q. Huang, K. Kaneko, K. Yokoi, S. Kajita, T. Kotoku, N. Koyachi, H. Arai, N. Imamura, K. Komoriya, and K. Tanie. Balance control of a biped robot combining off-line pattern with real-time modification. In Proc. 2000 IEEE Int. Conf. Robot. Autom. (ICRA), pages 3346-3352, San Francisco, April 2000.

Y. Hürmüzlü and D. B. Marghitu. Rigid body collisions of planar kinematic chains with multiple contact points. Int. J. Robot. Res., 13(1):82-92, 1994.

Y. Hürmüzlü, F. Génot, and B. Brogliato. Modeling, stability and control of biped robots - a general framework. Automatica, 40(10):1647-1664, 2004.

S. Hyon, J. G. Hale, and G. Cheng. Full-body compliant human-humanoid interaction: Balancing in the presence of unknown external forces. IEEE Trans. Robot., 23(5):884-898, 2007.

A. Isidori. Nonlinear Control Systems. Springer-Verlag, Berlin, third edition, 1995.

S. Jiang, S. Patrick, H. Zhao, and A. D. Ames. Outputs of human walking for bipedal robotic controller design. In Proc. Amer. Contr. Conf. (ACC), pages 4843-4848, Montreal, June 2012.

S. Kajita and K. Tani. Study of dynamic biped locomotion on rugged terrain-theory and basic experiment. In Int. Conf. Adv. Robot., pages 741-746, Pisa, June 1991.

S. Kajita and K. Tani. Adaptive gait control of a biped robot based on realtime sensing of the ground profile. Auton. Robots, 4(3):297-305, 1997.

S. Kajita, T. Yamaura, and A. Kobayashi. Dynamic walking control of a biped robot along a potential energy conserving orbit. IEEE Trans. Robot. Autom., 8 (4):431-438, 1992.

S. Kajita, F. Kanehiro, K. Kaneko, K. Yokoi, and H. Hirukawa. The 3D linear inverted pendulum mode: a simple modeling for a biped walking pattern generation. In Proc. 2001 IEEE/RSJ Int. Conf. Intell. Robots Syst. (IROS), volume 1, pages 239-246, Maui, October 2001.

S. Kajita, M. Morisawa, K. Harada, K. Kaneko, F. Kanehiro, K. Fujiwara, and H. Hirukawa. Biped walking 
pattern generation by using preview control of zeromoment point. In Proc. 2003 IEEE Int. Conf. Robot. Autom. (ICRA), volume 2, pages 1620-1626, Taipei, September 2003.

S. Kajita, M. Morisawa, K. Miura, S. Nakaoka, K. Harada, K. Kaneko, F. Kanehiro, and K. Yokoi. Biped walking stabilization based on linear inverted pendulum tracking. In Proc. 2010 IEEE/RSJ Int. Conf. Intell. Robots Syst. (IROS), pages 4489-4496, Taipei, October 2010.

K. Kaneko, F. Kanehiro, M. Morisawa, K. Akachi, G. Miyamori, A. Hayashi, and N. Kanehira. Humanoid robot HRP-4 - humanoid robotics platform with lightweight and slim body. In Proc. 2011 IEEE/RSJ Int. Conf. Intell. Robots Syst. (IROS), pages 4400-4407, San Francisco, 2011.

I. Kato, S. Ohteru, H. Kobayashi, K. Shirai, and A. Uchiyama. Information-power machine with senses and limbs. In First CISM-IFToMM Symp. Theory Prac. Robots and Manipulators, 5-8 September 1973, Udine, volume 2. Springer, New York, 1974.

H. K. Khalil. Nonlinear Systems. Prentice Hall, Upper Saddle River, 2nd edition, 1996.

J. Y. Kim, I. W. Park, and J. H. Oh. Walking control algorithm of biped humanoid robot on uneven and inclined floor. J. Intell. Robot. Syst., 48:457-484, 2007.

T. Kinugasa, C. Chevallereau, and Y. Aoustin. Effect of circular arc feet on a control law for a biped. Robotica, 27:621-632, 2009.

C. Kirtley, M. W. Whittle, and R. J. Jefferson. Influence of walking speed on gait parameters. J. Biomed. Eng., $7(4): 282-288,1985$.

S. Kolathaya and A. D. Ames. Achieving bipedal locomotion on rough terrain through human-inspired control. In IEEE Int. Symp. Safety, Security, and Rescue Robot., 2012.

S. Kolathaya, M. Pasupuleti, and A. D. Ames. Humaninspired underactuated bipedal robotic walking with AMBER on flat-ground, up-slope and uneven terrain. In Proc. 2011 IEEE/RSJ Int. Conf. Intell. Robots Syst. (IROS), pages 2478-2483, Loulé, October 2012.

T. Komura, A. Nagano, H. Leung, and Y. Shinagawa. Simulating pathological gait using the enhanced linear inverted pendulum model. Trans. Biomed. Eng., 52 (9):1502-1513, 2005.

T. Koolen, T. de Boer, J. Rebula, a. Goswami, and J. E. Pratt. Capturability-based analysis and control of legged locomotion, Part 1: Theory and application to three simple gait models. Int. J. Robot. Res., 31(9): 1094-1113, 2012.

P. Kormushev, B. Ugurlu, S. Calinon, N. G. Tsagarakis, and D. G. Caldwell. Bipedal walking energy minimization by reinforcement learning with evolving policy parameterization. In Proc. 2011 IEEE/RSJ Int. Conf. Intell. Robots Syst. (IROS), pages 318-324, San Francisco, 2011.

V. V. Kozlov and D. V. Treshchev. Billiards: A Genetic Introduction to the Dynamics of Systems with Impacts, volume 89 of Transl. Math. Monographs. AMS, Prov- idence, 1992.

S. Kudoh, T. Komura, and K. Ikeuchi. The dynamic postural adjustment with the quadratic programming method. In Proc. 2002 IEEE/RSJ Int. Conf. Intell. Robots Syst. (IROS), pages 2563-2568, Lausanne, October 2002.

A. D. Kuo. Stabilization of lateral motion in passive dynamic walking. Int. J. Robot. Res., 18(9):917-930, 1999.

A. D. Kuo. Energetics of actively powered locomotion using the simplest walking model. J. Biomed. Eng., 124:113-120, 2002.

A. D. Kuo. Choosing your steps carefully. IEEE Robot. Autom. Mag., 14(2):18-29, 2007.

R. Kurazume, T. Hasegawa, and K. Yoneda. The sway compensation trajectory for a biped robot. In Proc. 2003 IEEE Int. Conf. Robot. Autom. (ICRA), pages 925-931, Taipei, September 2003.

A. Lamperski and A. D. Ames. On the existence of Zeno behavior in hybrid systems with non-isolated Zeno equilibria. In Proc. 47th IEEE Conf. Decis. Contr., pages 2776-2781, Cancún, December 2008.

A. Lamperski and A.D. Ames. Lyapunov theory for Zeno stability. IEEE Trans. Autom. Contr., 58(1):100-112, 2013.

H.-O. Lim, Y. Kaneshima, and A. Takanishi. Online walking pattern generation for biped humanoid robot with trunk. In Proc. 2002 IEEE Int. Conf. Robot. Autom. (ICRA), volume 3, pages 3111-3116, Washington, D.C., May 2002.

J. Lygeros, K. H. Johansson, S. Simic, J. Zhang, and S. Sastry. Dynamical properties of hybrid automata. IEEE Trans. Autom. Contr., 48:2-7, 2003.

A. Majumdar, A. A. Ahmadi, and R. Tedrake. Control design along trajectories with sums of squares programming. In Proc. 2013 IEEE Int. Conf. Robot. Autom. (ICRA), pages 4054-4061, Karlsruhe, May 2013.

I. R. Manchester, U. Mettin, F. Iida, and R. Tedrake. Stable dynamic walking over uneven terrain. Int. J. Robot. Res., 30(3):265-279, 2011a.

I. R. Manchester, M. M. Tobenkin, M. Levashov, , and R. Tedrake. Regions of attraction for hybrid limit cycles of walking robots. In Proc. 18th IFAC World Cong., pages 5801-5806, Milan, August 2011b.

J. E. Marsden and T. S. Ratiu. Introduction to Mechanics and Symmetry, volume 17 of Texts in Applied Mathematics. Springer, 1999.

T. McGeer. Passive dynamic walking. Int. J. Robot. Res., 9(2):62-82, 1990a.

T. McGeer. Passive walking with knees. In Proc. 1990 IEEE Int. Conf. Robot. Autom. (ICRA), pages 16401645, Cincinnati, May 1990b.

T. A. McMahon and G. C. Cheng. The mechanics of running: How does stiffness couple with speed? J. Biomech., 23:65-78, 1990.

S. Miossec and Y. Aoustin. Walking gait composed of single and double supports for a planar biped without feet. In Proc. 5th Int. Conf. Climbing Walking Robots (CLAWAR), Paris, 25-27 September 2002, pages 767- 
$774,2002$.

S. Miossec and Y. Aoustin. Fast motions in Biomechanics and Robotics, chapter Dynamical synthesis of a walking cyclic gait for a biped with point feet, pages 233-252. LNCIS. Springer, Heidelberg, 2006.

H. Miura and I. Shimoyama. Dynamic walk of a biped. Int. J. Robot. Res., 3(2):60-74, 1984.

K. Miura, S. Nakaoka, M. Morisawa, K. Harada, and S. Kajita. A friction based twirl for biped robots. In 8th IEEE-RAS Int. Conf. Humanoid Robots (Humanoids), pages 279-284, Daejeon, 2008.

K. Miura, M. Morisawa, F. Kanehiro, S. Kajita, K. Kaneko, and K. Yokoi. Human-like walking with toe supporting for humanoids. In Proc. 2011 IEEE/RSJ Int. Conf. Intell. Robots Syst. (IROS), pages 4428-4435, San Francisco, September 2011. Ieee.

S. Mochon and T. A. McMahon. Ballistic walking. J. Biomech., 13(1):49-57, 1980.

J. J. Moreau. Quadratic programming in mechanics: Dynamics of one sided constraints. J. SIAM Contr., 4(1):153-158, 1966.

B. Morris and J. W. Grizzle. A restricted Poincaré map for determining exponentially stable periodic orbits in systems with impulse effects: Application to bipedal robots. In Proc. 44th IEEE Conf. Decis. Contr. and 2005 Euro. Contr. Conf. (CCC-ECC), pages 41994206, Seville, December 2005.

B. Morris and J. W. Grizzle. Hybrid invariant manifolds in systems with impulse effects with application to periodic locomotion in bipedal robots. IEEE Trans. Autom. Contr., 54(8):1751-1764, 2009.

B. Morris, M. J. Powell, and A. D. Ames. Sufficient conditions for the lipschitz continuity of QP-based multiobjective control of humanoid robots. In Proc. 52nd IEEE Conf. Decis. Contr., pages 2920-2926, Florence, December 2013.

Xiuping $\mathrm{Mu}$ and Qiong $\mathrm{Wu}$. On impact dynamics and contact events for biped robots via impact effects. Systems, Man, and Cybernetics, Part B: Cybernetics, IEEE Transactions on, 36(6):1364-1372, 2006.

R. M. Murray, Z. Li, and S. S. Sastry. A Mathematical Introduction to Robotic Manipulation. CRC Press, Boca Raton, 1993.

K. Nagasaka, H. Inoue, and M. Inaba. Dynamic walking pattern generation for a humanoid robot based on optimal gradient method. In IEEE Int. Conf. Syst., Man, and Cybernetics, volume 6, pages 908-913, Tokyo, October 1999.

S. G. Nersesov, V. Chellaboina, and W. M. Haddad. A generalization of Poincaré's theorem to hybrid and impulsive dynamical systems. Int. J. Hybrid Syst., 2: 35-51, 2002.

K. Nishiwaki, S. Kagami, Y. Kuniyoshi, M. Inaba, and H. Inoue. Online generation of humanoid walking motion based on a fast generation method of motion pattern that follows desired zmp. In Proc. 2002 IEEE/RSJ Int. Conf. Intell. Robots Syst. (IROS), volume 3, pages 2684-2689, Lausanne, October 2002.
M. Ogino, H. Toyama, and M. Asada. Stabilizing biped walking on rough terrain based on the compliance control. Proc. 2007 IEEE/RSJ Int. Conf. Intell. Robots Syst. (IROS), pages 4047-4052, October 2007.

Y. Ogura, H. Aikawa, K. Shimomura, A. Morishima, H.-O. Lim, and A. Takanishi. Development of a new humanoid robot WABIAN-2. In Proc. 2006 IEEE Int. Conf. Robot. Autom. (ICRA), pages 76-81, Orlando, 2006.

Y. Or and A. D. Ames. Stability of Zeno equlibria in Lagrangian hybrid systems. In Proc. 47th IEEE Conf. Decis. Contr., pages 2770-2775, Cancún, December 2008.

Y. Or and A. D. Ames. Existence of periodic orbits in completed lagrangian hybrid systems with nonplastic impacts. In R. Majumdar and P. Tabuada, editors, Proc. 12th Int. Conf. Hybrid Syst.: Comp. Contr.(HSCC), San Francisco, CA, 13-15 April 2009, volume 5469 of $L N C S$, pages 291-305, Berlin, 2009. Springer-Verlag.

H.-W. Park, K. Sreenath, J. W. Hurst, and J. W. Grizzle. Identification of a bipedal robot with a compliant drivetrain: Parameter estimation for control design. IEEE Contr. Syst. Mag., 31(2):63-88, April 2011.

H.-W. Park, K. Sreenath, A. Ramezani, and J. W. Grizzle. Switching control design for accommodating large step-down disturbances in bipedal robot walking. In Proc. 2012 IEEE Int. Conf. Robot. Autom. (ICRA), pages 45-50, St. Paul, May 2012.

H.-W. Park, A. Ramezani, and J. W. Grizzle. A finitestate machine for accommodating unexpected large ground-height variations in bipedal robot walking. IEEE Trans. Robot., 29(2):331-345, 2013.

I.-W. Park, J.-Y. Kim, J. Lee, and J.-H. Oh. Mechanical design of humanoid robot platform KHR-3 (KAIST Humanoid Robot 3: HUBO). In Proc. 5th IEEE-RAS Int. Conf. Humanoid Robots, pages 321-326, 2005.

J. H. Park and K. D. Kim. Biped robot walking using gravity-compensated inverted pendulum mode and computed torque control. In Proc. 1998 IEEE Int. Conf. Robot. Autom. (ICRA), pages 3528-3533, Leuven, May 1998.

T. S. Parker and L. O. Chua. Practical Numerical Algorithms for Chaotic Systems. Springer-Verlag, New York, 1989.

J. L. Patton, Y.-C. Pai, and W. A. Lee. Evaluation of a model that determines the stability limits of dynamic balance. Gait \& Posture, 9(1):38-49, 1999.

J. Perry and J. Burnfield. Gait Analysis: Normal and Pathological Function. Slack Inc., Thorofare, 2 edition, 2010.

I. R. Petersen and B. R. Barmish. Control effort considerations in the stabilization of uncertain dynamical systems. Syst. Contr. Let., 9(5):417-422, 1987.

F. Pfeiffer, K. Loffler, and M. Gienger. The concept of jogging Johnnie. In Proc. 2002 IEEE Int. Conf. Robot. Autom. (ICRA), pages 3129-3135, Washington, D.C., May 2002.

F. Plestan, J. W. Grizzle, E. R. Westervelt, and G. Abba. 
Stable walking of a 7-DOF biped robot. IEEE Trans. Robot., 19(4):653-668, August 2003.

M. B. Popovic, A. Goswami, and H. Herr. Ground reference points in legged locomotion: Definitions, biological trajectories and control implications. Int. J. Robot. Res., 24(12):1013-1032, 2005.

M. Posa, C. Cantu, and R. Tedrake. A direct method for trajectory optimization of rigid bodies through contact. Int. J. Robot. Res., 33(1):69-81, 2014.

I. Poulakakis and J. W. Grizzle. Monopedal running control: SLIP embedding and virtual constraint controllers. In Proc. 2007 IEEE/RSJ Int. Conf. Intell. Robots Syst. (IROS), pages 323-330, San Diego, 2007.

I. Poulakakis and J. W. Grizzle. The spring loaded inverted pendulum as the hybrid zero dynamics of an asymmetric hopper. IEEE Trans. Autom. Contr., 54 (8):1779-1793, 2009.

M. J. Powell, A. Hereid, and A. D. Ames. Speed regulation in $3 \mathrm{D}$ robotic walking through motion transitions between human-inspired partial hybrid zero dynamics. In Proc. 2013 IEEE Int. Conf. Robot. Autom. (ICRA), pages 4803-4810, Karlsruhe, 2013.

G. Pratt. Legged robots at MIT: What's new since Raibert? IEEE Robot. Autom. Mag., September 2000.

J. E. Pratt and S. V. Drakunov. Derivation and application of a conserved orbital energy for the inverted pendulum bipedal walking model. In Proc. 2007 IEEE Int. Conf. Robot. Autom. (ICRA), pages 4653-4660, Rome, 2007.

J. E. Pratt and G. Pratt. Exploiting natural dynamics in the control of 3D bipedal walking simulation. In G. S. Virk, M. J. Randall, and D. Howard, editors, Proc. 2nd Int. Conf. Climbing Walking Robots (CLAWAR), Portsmouth, 14-15 September 1999, pages 797-807, Hoboken, 1999. Wiley-Blackwell.

J. E. Pratt and R. Tedrake. Velocity-based stability margins for fast bipedal walking. In Fast Motions in Biomechanics and Robotics, pages 299-324. Springer, 2006.

J. E. Pratt, M. C. Chee, A. Torres, P. Dilworth, and G. Pratt. Virtual model control: an intuitive approach for bipedal locomotion. Int. J. Robot. Res., 20(2):129143, 2001.

J. E. Pratt, J. Carff, S. Drakunov, and A. Goswami. Capture point: A step toward humanoid push recovery. In 6th IEEE-RAS Int. Conf. Humanoid Robots (Humanoids), pages 200-207, Genoa, December 2006.

J. E. Pratt, T. Koolen, T. de Boer, J. Rebula, S. Cotton, J. Carff, M. Johnson, and P. Neuhaus. Capturabilitybased analysis and control of legged locomotion, Part 2: Application to M2V2, a lower-body humanoid. Int. J. Robot. Res., 31(10):1117-1133, 2012.

M. H. Raibert. Hopping in legged systems - modeling and simulation for the two-dimensional one-legged case. IEEE Trans. Systems, Man, Cybernetics, 14(3): 451-463, 1984.

M. H. Raibert. Legged robots that balance. MIT Press, Cambridge, 1986.

M. H. Raibert. Trotting, pacing and bounding by a quadruped robot. J. Biomech., 23(1):79-98, 1990.

M. H. Raibert and H. Brown, Jr. Experiments in balance with a $2 \mathrm{D}$ one-legged hopping machine. ASME J. Dynamic Syst., Measurement, Contr., 106:75-81, 1984.

M. H. Raibert, H. B. Brown, and S. S. Murthy. 3D balance using 2D algorithms? In M. Brady and R. Paul, editors, Robot. Res.: First Int. Symp., volume 12 of Artificial Intelligence, pages 279-301. MIT Press, 1984.

M. H. Raibert, M. Chepponis, and H. Brown Jr. Running on four legs as though they were one. J. Robot. Autom., 2(2):70-82, 1986.

A Ramezani, J. W. Hurst, K. Akbari Hamed, and J. W. Grizzle. Performance analysis and feedback control of ATRIAS, a three-dimensional bipedal robot. J. Dynamic Syst., Measurement, Contr., 136(2):02101121-0210112-12, March 2014.

J. Rebula, F. Canas, J. E. Pratt, and A. Goswami. Learning capture points for humanoid push recovery. In 7th IEEE-RAS Int. Conf. Humanoid Robots (Humanoids), pages 65-72, Pittsburgh, November 2007.

Adrian Rodriguez and Alan Bowling. Solution to indeterminate multipoint impact with frictional contact using constraints. Multibody System Dynamics, 28(4): 313-330, 2012.

Adrian Rodriguez and Alan Bowling. Study of the stickslip transition of newton's cradle with friction. In ASME 2013 International Design Engineering Technical Conferences and Computers and Information in Engineering Conference, pages V07AT10A044V07AT10A044. American Society of Mechanical Engineers, 2013.

A. V. Roup, D. S. Bernstein, S. G. Nersesov, W. M. Haddad, and V. Chellaboina. Limit cycle analysis of the verge and foliot clock escapement using impulsive differential equations and Poincare maps. Int. J. Contr., 76(17):1685-1698, 2003.

N. Sadati, G. A. Dumont, K. A. Hamed, and W. A. Gruver. Hybrid Control and Motion Planning of Dynamical Legged Locomotion. IEEE Press Series on Systems Science and Engineering. Wiley, 2012.

Y. Sakagami, R. Watanabe, C. Aoyama, S. Matsunaga, N. Higaki, and K. Fujimura. The intelligent ASIMO: system overview and integration. In Proc. 2002 IEEE/RSJ Int. Conf. Intell. Robots Syst. (IROS), pages 2478-83, Lausanne, September 2002.

U. Saranli, M. Buehler, and D. E. Koditschek. RHex: A simple and highly mobile hexapod robot. Int. J. Robot. Res., 20(7):616-631, 2001.

S. S. Sastry. Nonlinear Systems: Analysis, Stability and Control. Springer, New York, 1999.

L. Sciavicco and B. Siciliano. Modeling and Control of Robot Manipulators. McGraw-Hill, New York, 1996.

A. Seyfarth, H. Geyer, M. Gunther, and R. Blickhan. A movement criterion for running. J. Biomech., 35: 649-655, 2001.

C.-L. Shih. Ascending and descending stairs for a biped robot. Trans. Syst., Man, Cybernetics, Part A, 29(3): 255-268, 1999.

C.-L. Shih and C.-J. Chiou. The motion control of a stat- 
ically stable biped robot on an uneven floor. Trans. Syst., Man, Cybernetics, Part B, 28(2):244-249, 1998.

H. Shimizu, Y. Wakazuki, Y. Pan, and K. Furuta. Biped walking robot using a stick on uneven ground. In SICE Annual Conf. 2007, pages 83-8, Takamatsu, September 2007.

B. Siciliano and O. Khatib. Springer Handbook of Robotics. Springer, 2008.

B. Siciliano and L. Villani. Robot Force Control. Kluwer Academic Publishers, Hingham, 1999.

R. W. Sinnet. Hybrid geometric feedback control of three-dimensional bipedal robotic walkers with knees and feet. Master's thesis, Texas A\&M University, May 2011.

R. W. Sinnet and A. D. Ames. 2D bipedal walking with knees and feet: A hybrid control approach. In Proc. 48th IEEE Conf. Decis. Contr. and 28th Chinese Contr. Conf. (CDC/CCC), pages 3200-3207, Shanghai, December 2009a.

R. W. Sinnet and A. D. Ames. 3D bipedal walking with knees and feet: A hybrid geometric approach. In Proc. 48th IEEE Conf. Decis. Contr. and 28th Chinese Contr. Conf. (CDC/CCC), pages 3208-3213, Shanghai, December 2009b.

R. W. Sinnet and A. D. Ames. Extending twodimensional human-inspired bipedal robotic walking to three dimensions through geometric reduction. In Proc. Amer. Contr. Conf. (ACC), pages 4831-4836, Montreal, June 2012a.

R. W. Sinnet and A. D. Ames. Bio-inspired feedback control of three-dimensional humanlike bipedal robots. $J$. Robot. Mechatronics, 24(4):595-601, 2012b.

R. W. Sinnet, M. J. Powell, R. P. Shah, and A. D. Ames. A human-inspired hybrid control approach to bipedal robotic walking. In Proc. 18th IFAC World Cong., pages 6904-6911, Milan, August 2011.

E. Sontag. A 'universal' contruction of Artstein's theorem on nonlinear stabilization. Syst. Contr. Let., 13: 117-123, 1989.

M. W. Spong. Modeling and control of elastic joint robots. J. Dynamic Syst., Measurement, Contr., 109 (4):310-309, 1987.

M. W. Spong. Passivity based control of the compass gait biped. In Proc. 6th IFAC World Cong., pages 1924, Beijing, July 1999.

M. W. Spong and F. Bullo. Controlled symmetries and passive walking,. IEEE Trans. Autom. Contr., 50(7): 1025-1031, 2005.

M. W. Spong, S. Hutchinson, and M. Vidyasagar. Robot Modeling and Control. John Wiley \& Sons, 2005.

K. Sreenath, H.-W. Park, and J. W. Grizzle. Embedding active force control within the compliant hybrid zero dynamics to achieve stable, fast running on MABEL. Int. J. Robot. Res., 2011a.

K. Sreenath, H.-W. Park, I. Poulakakis, and J. W. Grizzle. A compliant hybrid zero dynamics controller for stable, efficient and fast bipedal walking on MABEL. Int. J. Robot. Res., 30(9):1170-1193, 2011b.

K. Sreenath, H.-W. Park, and J. W. Grizzle. Design and experimental implementation of a compliant hybrid zero dynamics controller with active force control for running on MABEL. In Proc. 2012 IEEE Int. Conf. Robot. Autom. (ICRA), pages 51-56, St. Paul, May 2012.

M. Srinivasan and A. Ruina. Computer optimization of a minimal biped model discovers walking and running. Nature, 439:72-75, January 2006.

S. Srinivasan, I. A. Raptis, and E. R. Westervelt. A lowdimensional sagittal plane model of normal human walking. J. Biomed. Eng., 130(5):051017, 2008.

S. Srinivasan, E. R. Westervelt, and A. H. Hansen. A lowdimensional sagittal-plane forward-dynamic model for asymetric gait and its application to study the gait of transtibial prosthesis users. J. Biomed. Eng., 131(3): 031003, 2009.

B. Stephens. Humanoid push recovery. In 7th IEEERAS Int. Conf. Humanoid Robots (Humanoids), pages 589-595, Pittsburgh, November 2007.

B. Stephens. Push Recovery Control for Force-Controlled Humanoid Robots. PhD thesis, Carnegie Mellon University, 2011.

B. J Stephens and C. G. Atkeson. Push recovery by stepping for humanoid robots with force controlled joints. In 10th IEEE-RAS Int. Conf. Humanoid Robots (Humanoids), pages 52-59, December 2010.

J. L. Su and J. B. Dingwell. Dynamic stability of passive dynamic walking on an irregular surface. Trans. ASME, 129:802-810, 2007.

A. Takanishi, M. Ishida, Y. Yamazaki, and I. Kato. The realization of dynamic walking by the biped walking robot wl-10rd. In Int. Conf. Adv. Robot., pages 459466, Tokyo, September 1985.

A. Takanishi, T. Takeya, H. Karaki, and I. Kato. A control method for dynamic biped walking under unknown external force. In Proc. 1990 IEEE/RSJ Int. Conf. Intell. Robots Syst. (IROS), volume 2, pages 795-801, Ibaraki, July 1990.

T. Takenaka, T. Matsumoto, and T. Yoshiike. Real time motion generation and control for biped robot - 3rd report: Dynamics error compensation. In Proc. 2009 IEEE/RSJ Int. Conf. Intell. Robots Syst. (IROS), pages 1594-1600, St. Louis, October 2009.

R. Tedrake, I. R. Manchester, M. M. Tobenkin, and J. W. Roberts. LQR-trees: Feedback motion planning via sums of squares verification. Int. J. Robot. Res., 29: 1038-1052, 2010.

R. Vasudevan, A. D. Ames, and R. Bajcsy. Persistent homology for automatic determination of human-data based cost of bipedal walking. Nonlinear Analysis: Hybrid Systems, 7(1):101 - 115, 2013.

M. Vukobratović and B. Borovac. Zero-moment point Thirty five years of its life. Int. J. Humanoid Robot., 1(1):157-173, 2004.

M. Vukobratović, B. Borovac, D. Surla, and D. Stokic. Biped Locomotion. Springer-Verlag, Berlin, 1990.

M. Vukobratović, B. Borovac, and V. Potkonjak. ZMP: A review of some basic misunderstandings. Int. J. Humanoid Robot., 3(2):153-175, 2006. 
T. Wang, C. Chevallereau, and C.F. Rengifo. Walking and steering control for a 3D biped robot considering ground contact and stability. Robot. Auton. Syst., 60 (7):962-977, 2012.

T. Wang, C. Chevallereau, and D. Tlalolini. Stable walking control of a 3D biped robot with foot rotation. Robotica, FirstView:1-20, March 2014.

Q. F. Wei, P. S. Krishnaprasad, and W. P. Dayawansa. Modeling of impact on a flexible beam. In Proc. 32nd IEEE Conf. Decis. Contr. (CDC), pages 1377-1382, San Antonio, December 1993.

E. R. Westervelt, J. W. Grizzle, and D. E. Koditschek. Hybrid zero dynamics of planar biped walkers. IEEE Trans. Autom. Contr., 48(1):42-56, 2003.

E. R. Westervelt, G. Buche, and J. W. Grizzle. Experimental validation of a framework for the design of controllers that induce stable walking in planar bipeds. Int. J. Robot. Res., 24(6):559-582, 2004.

E. R. Westervelt, J. W. Grizzle, C. Chevallereau, J.-H. Choi, and B. Morris. Feedback Control of Dynamic Bipedal Robot Locomotion. CRC Press, Boca Raton, $2007 \mathrm{a}$.

E. R. Westervelt, B. Morris, and K. D. Farrell. Analysis results and tools for the control of planar bipedal gaits using hybrid zero dynamics. Auton. Robots, 23(2): 131-145, 2007b.

D. A. Winter. Human balance and posture control during standing and walking. Gait $\mathscr{E}$ Posture, 3(4):193214, 1995.

D. A. Winter. Biomechanics and Motor Control of Human Movement. John Wiley \& Sons, 4 edition, 2009.

M. Wisse and R. Q. van der Linde. Delft Pneumatic Bipeds, volume 34 of Springer Tracts in Advanced Robotics. Springer-Verlag, Berlin, 2007.

S. Nadubettu Yadukumar, M. Pasupuleti, and A. D. Ames. From formal methods to algorithmic implementation of human inspired control on bipedal robots. In E. Frazzoli, T. Lozano-Perez, N. Roy, and D. Rus, editors, Algorithmic Foundations of Robotics $X$, volume 86 of Tracts in Advanced Robotics, pages 511-526. Springer, 2013.

M. Yagi and V. Lumelsky. Synthesis of turning pattern trajectories for a biped robot in a scene with obstacles. In Proc. 2000 IEEE/RSJ Int. Conf. Intell. Robots Systems (IROS), pages 1161-1166, Takamatsu, 2000.

J. Yamaguchi, A. Takanishi, and I. Kato. Experimental development of a foot mechanism with shock absorbing material for acquisition of landing surface position information and stabilization of dynamic biped walking. In Proc. 1995 IEEE Int. Conf. Robot. Autom. (ICRA), pages 2892-2899, Nagoya, May 1995.

J. Yamaguchi, E. Soga, S. Inoue, and A. Takanishi. Development of a bipedal humanoid robot-control method of whole body cooperative dynamic biped walking. In Proc. 1999 IEEE Int. Conf. Robot. Autom. (ICRA), pages 368-374, Detroit, May 1999.

T. Yang, E. R Westervelt, and A. Serrani. A framework for the control of stable aperiodic walking in underactuated planar bipeds. In Proc. 2007 IEEE Int. Conf.
Robot. Autom. (ICRA), pages 4661-4666, Rome, 2007. T. Yang, E. R. Westervelt, A. Serrani, and J. P. Schmiedeler. A framework for the control of stable aperiodic walking in underactuated planar bipeds. Auton. Robots, 27(3):277-290, 2009.

H. Ye, A. N. Michel, and L. Hou. Stability theory for hybrid dynamical systems. IEEE Trans. Autom. Contr., 43(4):461-474, 1998.

K. Yin, K. Loken, and M. van de Panne. SIMBICON: Simple Biped Locomotion Control. ACM Trans. Graphics (TOG), 26(3):105.1-105.10, 2007.

G. Zeglin and H. Brown. Control of a bow leg hopping robot. In Proc. 1998 IEEE Int. Conf. Robot. Autom. (ICRA), pages 793-798, Leuven, May 1998.

F. Zhang. The Schur Complement and Its Applications. Springer, New York, 2005. 